\title{
Effects of BMP15 and GDF9 on Ovine Oocytes in an In Vitro Maturation System
}

Thesis submitted in partial fulfilment of the requirements for the degree of Master of

Science in Biotechnology

\section{Aanchal Radhika Singh}

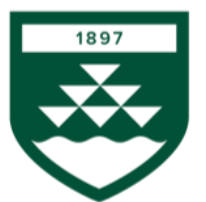

VICTORIA UNIVERSITY OF WELLINGTON

TE HERENGA WAKA

School of Biological Sciences

Victoria University of Wellington

Te Herenga Waka

2020 





\section{Abstract}

Oocyte developmental competency is the intrinsic measure of oocyte quality and the capacity for a mature oocyte to support the early stages of embryo development and implantation. Oocyte-secreted factors (OSFs), such as growth differentiation factor 9 (GDF9) and bone morphogenetic protein 15 (BMP15), play a pivotal role in regulating the synchrony of various complex maturation events within the cumulus-oocyte complex (COC) through the induction of paracrine and endocrine signalling. These proteins act synergistically to influence the proliferation and differentiation of granulosa cells (GCs), cumulus cell (CC) expansion, promote survival, ovulation, the attainment of developmental competency and fertility. Species-specific ratios suggest that poly-ovulatory mammals have increased fecundity due to high ratios of GDF9:BMP15, which is directly reflected in their large litter size. Interestingly, it has also been found that higher ratios of GDF9:BMP15 also increased blastocyst rate in sheep implying that these embryos develop from oocytes that are more developmentally competent.

In this study, I investigated the hypothesis that supplementing a commercial in vitro maturation (IVM) system with a high ratio of GDF9:BMP15 would increase the developmental competency sheep oocytes; a species with low-moderate litter size. To test this hypothesis, ovine oocytes were matured in a biphasic IVM system containing GDF9 and BMP15 at three divergent ratios $(1: 6,1: 1,6: 1)$. The results herein show that the $6: 1$ ratio resulted in higher levels of reagent transfer to the ovine oocyte through gap junctions (GJs) after 24 hours of incubation. Similarly, it was also observed that at the higher ratio, glutathione (GSH) levels were higher at 7.5 hours of incubation. The high GDF9:BMP15 ratio also facilitated the increased consumption of pyruvate by the COC consistently throughout the culture period. Importantly, the high GDF9:BMP15 ratio showed higher expression of the gene that encodes GJ (CX43) at 24 hours relative to the control. It was also demonstrated through decreased apoptotic factor (BAX:BCL2) ratios, that the addition of OSFs, regardless of ratio, protected against cell death. In summary, this study provides novel results that support the notion that a high GDF9:BMP15 ratio improves oocyte quality by delaying the timing of meiotic resumption. This subsequently improves the transport of key metabolites and antioxidants to protect against oxidative stress and cell death and aid in the completion of maturation, ultimately resulting in the increased developmental competency observed in high fecundity poly-ovulatory species. 


\section{Acknowledgements}

First and foremost, I would like to give my most heartfelt thanks to Dr. Janet Pitman for all your wisdom and assistance over the course of my Masters, and especially for always seeing my results in a positive light especially when I could not. I will be eternally grateful that you took me on as a student two years ago and for making me bloom into the young scientist I have become all thanks to your constant kindness, hard work and dedication. You are such an inspiration to all women in science.

Thank you Matire, Mel, Sarah, Odey, Hamish, Abbie and Matilda for helping me with my experiments and being especially accommodating to unavoidable cancellations. I could not have achieved what I did without your help and constant support. Thank you James and the team at Assure Quality for providing us with ovaries and also being accommodating with cancellations and last-minute orders, this study would literally not have happened without you all.

Thank you to the Pitman Lab (past and present): Janet, Matire, Mel, Sarah, Odey, Hamish, Zak, Frey, Orin, Shalen, Balam, Tessa, Charlotte, Latarsha, Peter and Emma for teaching me the ways of the lab, putting up with my questions, tears, ordering things for me (apparently I am expensive), catching me when I fall (quite literally) and just for the laughs and banter especially after spending hours down in the basement lab. You all have been the greatest support system and I loved having you all as my second family for the past two years. You guys are so brilliant and are already making such a huge contribution to the scientific community.

Thank you to Victoria University for their Masters by Thesis Scholarship as this has helped me focus on my research without stressing about my financial situation in the past year.

Lastly, I would like to thank my peers, my friends and my family. The past two years have not been easy and I am so grateful to the students and staff in SBS for helping me keep my sanity. Thank you Grace and Brittany, you guys were there for me on some of my ugliest and hardest days, and even providing me with a roof over my head on some nights. You both are absolute gems and I hope that I can do half of what you have done for me one day, and I am so grateful for our friendship. Thank you Janine, Jess, Daniel and Tory (and many others) for keeping me company when I was working long hours or during the 
weekend, for making sure I was okay, for the laughs, car rides, hugs, tea times, dealing with my tears, for letting me rant, and for simply making sure that I was taking a break and eating. Thank you Nina and Alicia for being patient when I could not be there for you, but for cheering me on regardless - you girls are the greatest friends in the entire world and I am so glad that you both have been a constant in my life for the past decade. And thank you to my immediate family - mum, dad, Arisha, Nishi, Maciek and little Rohan. Mum and dad - thank you for feeding me, dropping me off and picking me up from campus even in the weekend no matter the hour, financially supporting me during some very difficult times and just letting me focus on my studies. Nothing I could do will ever repay you both for the sacrifices you have made for our education, the fulfilment of our dreams and our happiness. I love you both very much and I hope that our achievements have made it all worth it. 


\section{Table of Contents}

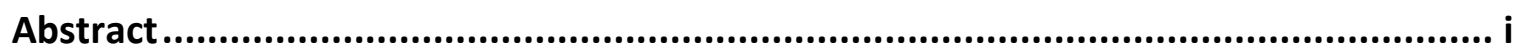

Acknowledgements.....................................................................................................ii

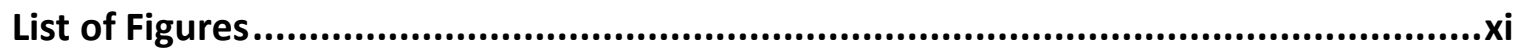

List of Tables.............................................................................................................. xiii

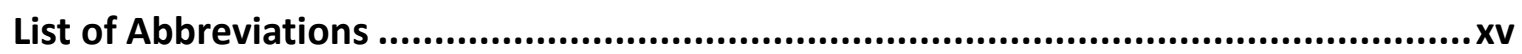

CHAPTER 1: INTRODUCTION ............................................................................ 1

1.1. In Vitro Production \& Oocyte Developmental Competency................................1

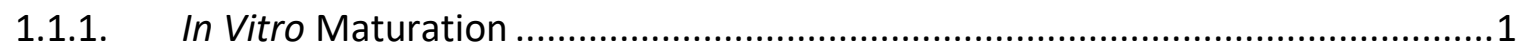

1.2. Follicular Development in the Ewe .........................................................

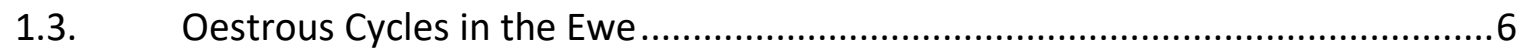

1.4. Maturation \& the Acquisition of Developmental Competency ............................

1.4.1. Nuclear Maturation ...................................................................................

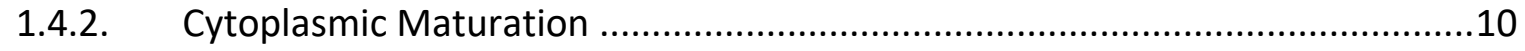

1.4.3. Metabolic Influence in Follicular Maturation ...................................................14

1.4.4. Ovulation \& Developmental Competency ......................................................18

1.5. Bone Morphogenetic Protein 15 \& Growth Differentiation Factor 9................21

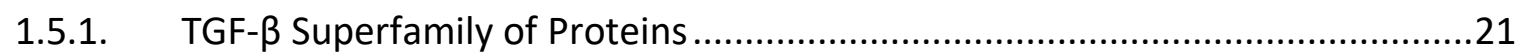

1.5.2. Receptors of the TGF- $\beta$ Superfamily ............................................................21

1.5.3. Molecular Characteristics of GDF9 \& BMP15 ...............................................22

1.5.4. Role of GDF9 \& BMP15 in Somatic Cell Function ...........................................23

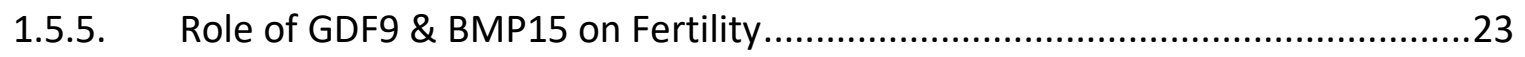

1.5.6. Effects of Covalently-linked Dimers on Biological Functions.............................25

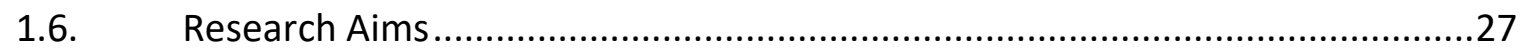

CHAPTER 2: MATERIALS \& METHODS

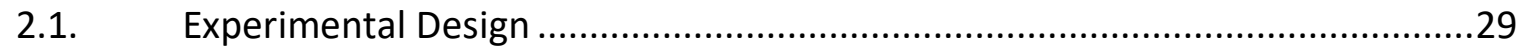

2.2. Biphasic Gold Standard Ovine IVM System …………………………..........30

2.2.1. IVM Medium with GDF9:BMP15 Supplementation.........................................31

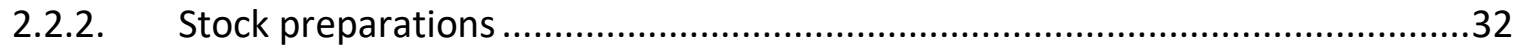




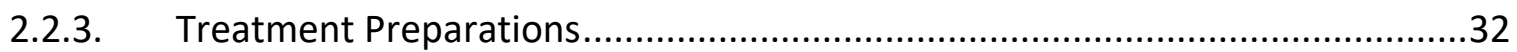

2.2.4. Ovary Dissection and Primary Cell Collection .................................................33

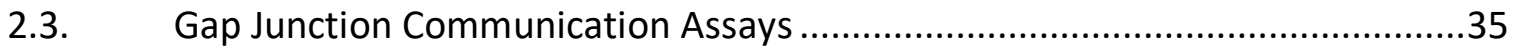

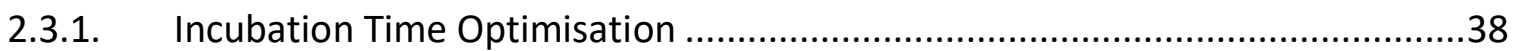

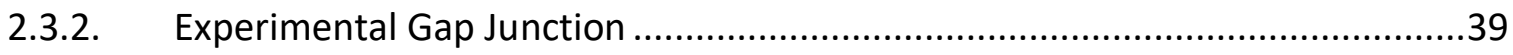

2.4. Glutathione Conjugation Assay for Oxidative Metabolism ............................41

2.5. Morphological Assessment for Maturation ......................................................45

2.5.1. Germinal Vesicle Breakdown \& Meiotic Resumption......................................45

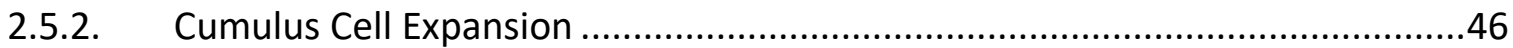

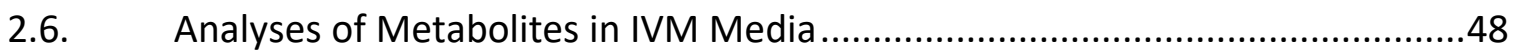

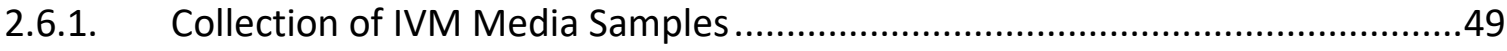

2.6.2. Optimising for the Detection of Pyruvate in IVM Media ...................................49

2.6.3. Detection of Pyruvate Consumption in IVM Medium .....................................50

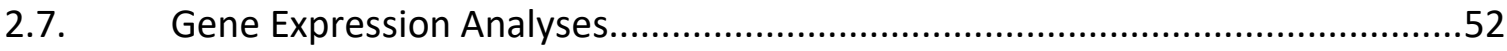

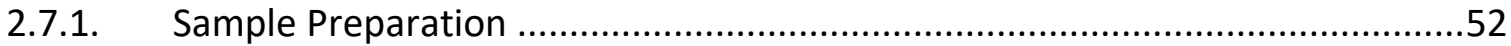

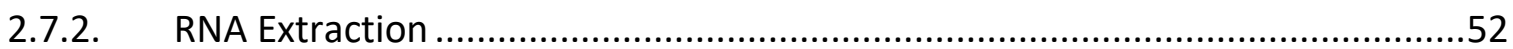

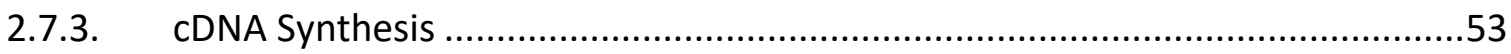

2.7.4. Optimisation of Gene Biomarkers for Developmental Competency.................53

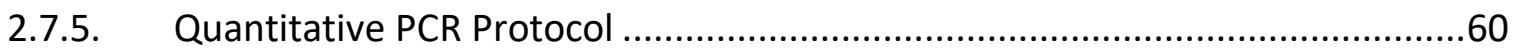

2.8. Granulosa Cell Proliferation Assay .................................................................61

2.8.1. Preparation of Granulosa Cell Incubation Media .........................................61

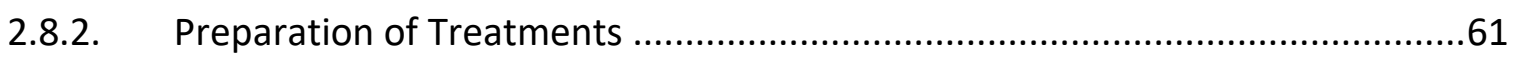

2.8.3. Granulosa Cell Collection \& Preparation ........................................................62

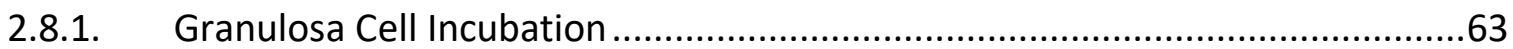

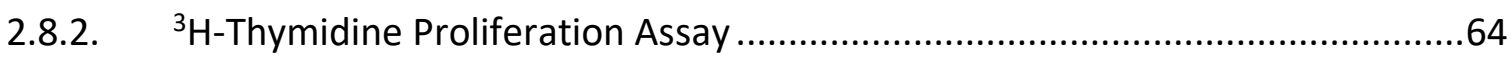

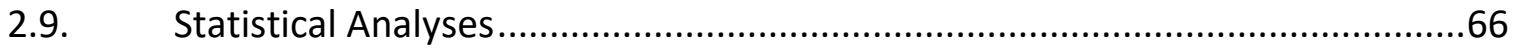

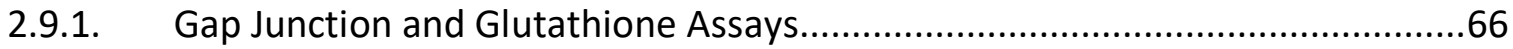

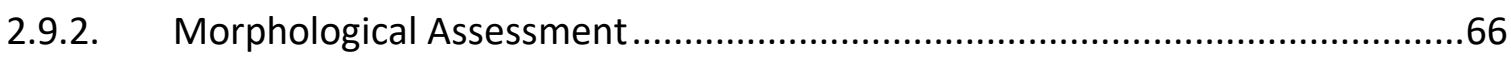

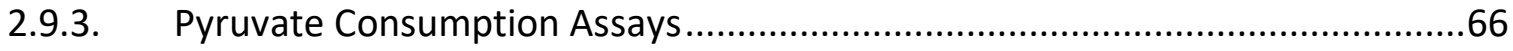

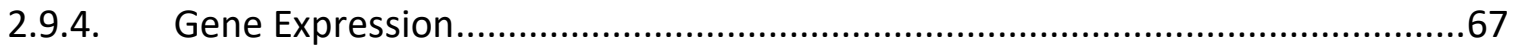

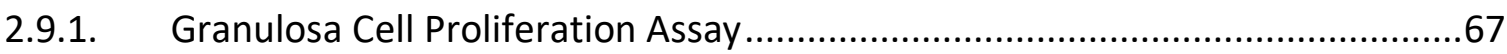


3.1. Gap Junction Communication Assays .........................................................69

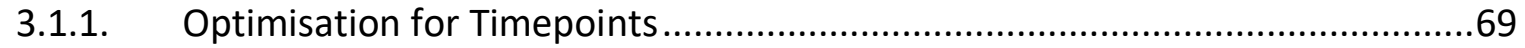

3.1.2. Experimental Gap Junction Communication ................................................

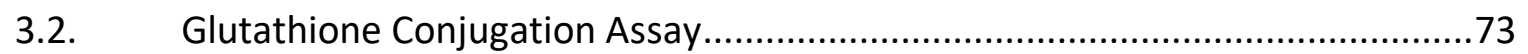

3.3. Morphological Assessment of Maturation .....................................................75

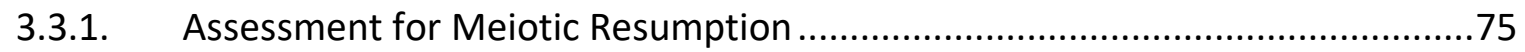

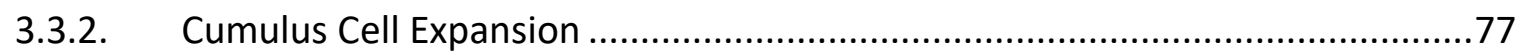

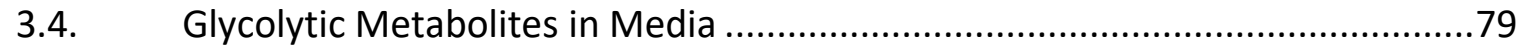

3.4.1. Optimisation of Pyruvate Consumption Bioassay ….......................................79

3.4.2. Pyruvate Consumption by Ovine COC ......................................................... 81

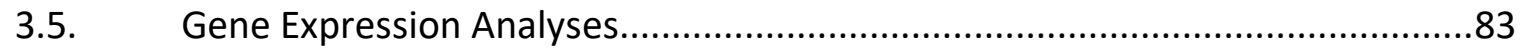

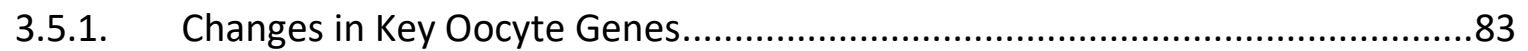

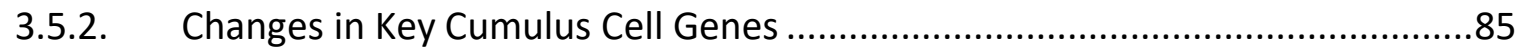

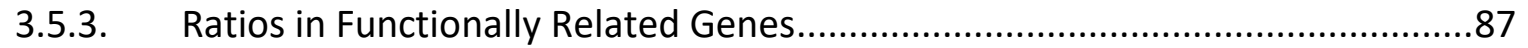

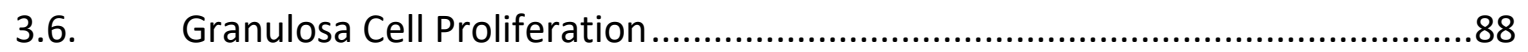

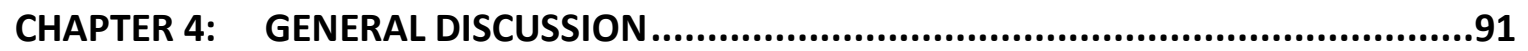

4.1. Effect of GDF9 \& BMP15 on Cytoplasmic Maturation in IVM ..........................93

4.1.1. The Effect of GDF9 \& BMP15 on Metabolite Consumption ............................93

4.1.2. Effect of GDF9 \& BMP15 on Oxidative Stress .................................................94

4.2. Effect of GDF9 \& BMP15 on Nuclear Maturation Networks .........................100

4.3. Effect of GDF9 \& BMP15 on the Completion of Maturation Events ...............103

4.4. Effects of GDF9 \& BMP15 on GC Proliferation ............................................106

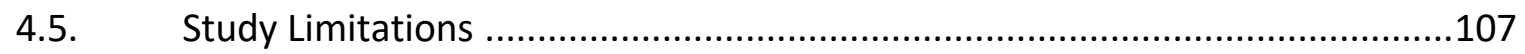

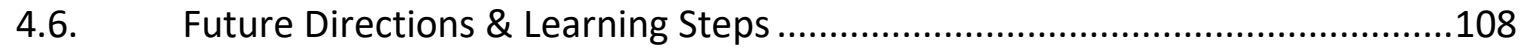

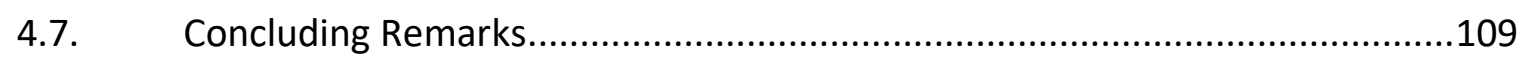

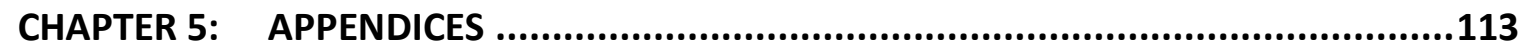

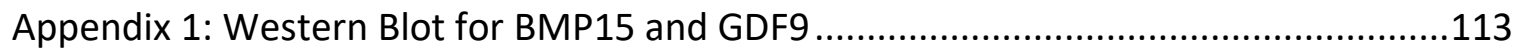

Appendix 2: Stocks, Solutions \& Media Recipes ........................................................115

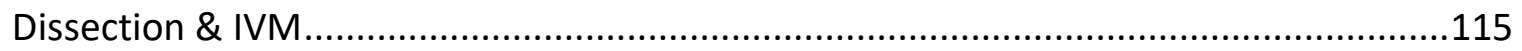


Gap Junction Assays

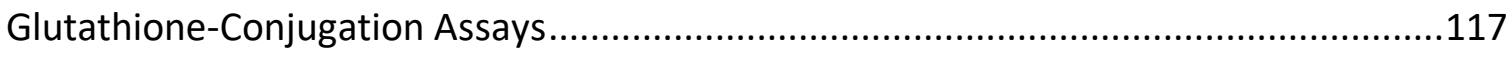

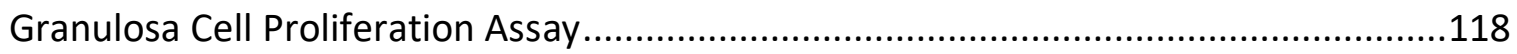

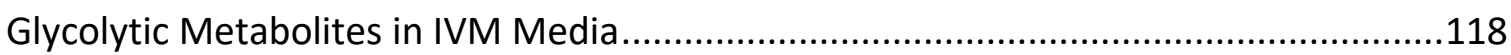

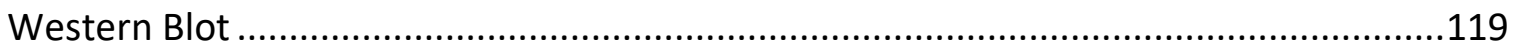

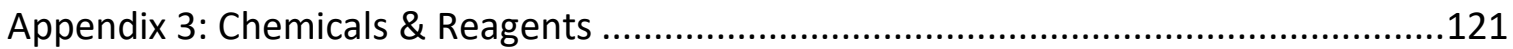

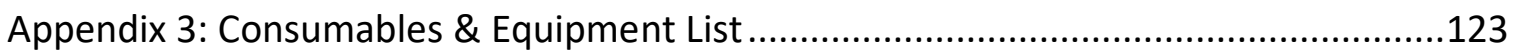

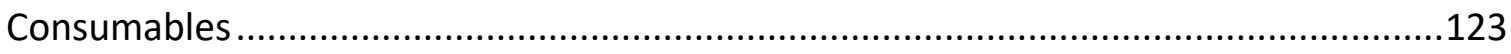

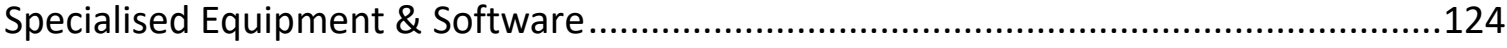

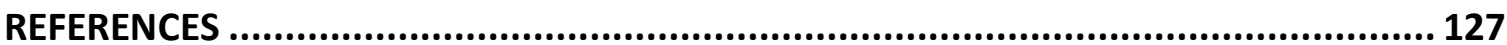




\section{List of Figures}

Figure 1: Depiction of bidirectional communication within the ovarian follicle..............3

Figure 2: Follicular development from a primordial to tertiary stage. ..........................6

Figure 3: The molecular overview of maintaining meiotic arrest and nuclear

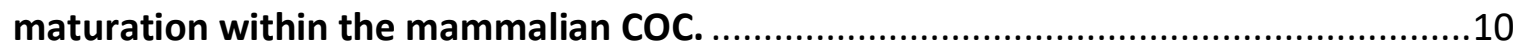

Figure 4: Modifications to Gold Standard IVM incubation system. ..............................31

Figure 5: Experimental overview for gap junction communication assay.....................36

Figure 6: Schematic diagram of gap junction communication that indirectly measures

communication between $\mathrm{CC}$ and oocyte.

Figure 7: Microscopic images of bovine cumulus-oocyte complexes (COC) and denuded oocyte before and after exposure to calcein-AM.

Figure 8: Fluorescence levels in ovine denuded oocytes as an indirect level of bidirectional intra-oocyte communication via GJs. .40

Figure 9: Experimental overview for glutathione assay.

Figure 10: Schematic diagram depicting the conjugation of monochlorobimane (MCB) to reduced glutathione (GSH).

Figure 11: Fluorescence levels in ovine denuded oocyte as an indirect measure of reduced glutathione (GSH) in the ooplasm.

Figure 12: Germinal vesicle (GV) and germinal vesicle breakdown (GVBD) in ovine denuded oocyte.

Figure 13: Germinal Vesicle (GV) in ovine denuded oocyte with and without probe staining.

Figure 14: Non-expanded and expanded cumulus cells (CCs).

Figure 15: Granulosa Cell (GC) morphology.

Figure 16: Effect of time on gap junction (GJ) communication in ovine $\mathrm{COC}$ in a biphasic IVM culture system.

Figure 17: Fold change of gap junction (GJ) communication, represented by intraoocyte fluorescence levels, in ovine COC incubated in an IVM culture system over 24 hours.

Figure 18: Effects of different ratios of GDF9 and BMP15 in a biphasic IVM culture system on gap junction (GJ) communication in ovine COC. 
Figure 19: Fold change of gap junction (GJ) communication, represented by intraoocyte fluorescence levels, in ovine COC incubated in an IVM culture system supplemented with different ratios of GDF9 and BMP15.

Figure 20: Effect of different ratios of GDF9 and BMP15 in a biphasic IVM culture system on the conjugation levels of lipophilic molecules to reduced glutathione (GSH) in ovine COC.

Figure 21: Fold-change of conjugation levels of lipophilic molecules to reduced glutathione (GSH) in ovine COC exposed to different ratios of GDF9 and BMP15 in a biphasic IVM culture system.

Figure 22: Sodium pyruvate standards tested in phenol-red and phenol-red free conditions to establish a standard curve.

Figure 23: Sodium pyruvate standards tested in fresh and freeze-thawed enzyme assay mix to establish a standard curve.

Figure 24: Effect of different ratios of GDF9 and BMP15 in a biphasic IVM culture system on the rate of pyruvate consumption by ovine COC.

Figure 25: Effect of GDF9 and BMP15 ratios on relative mRNA expression levels in ovine oocytes following incubation in a biphasic IVM culture system.

Figure 26: Effect of GDF9 and BMP15 ratios on relative mRNA expression levels in ovine cumulus cells (CC) following incubation in a biphasic IVM culture system.

Figure 27: Effect of GDF9 and BMP15 ratios on relative mRNA expression levels on BAX:BCL2 in ovine cumulus cells (CC) following incubation in a biphasic IVM culture system.

Figure 28: Effect of GDF9 and BMP15 ratios on the proliferation of granulosa cells (GC).

Figure 29: Effect of GDF9 and BMP15 ratios on the fold-change in proliferation of granulosa cells (GC).

Figure 30: Western Blot of different batches of in house pBMP15.

Figure 31: Western Blot of different batches of in house pGDF9. 


\section{List of Tables}

Table 1: Table of biomarkers selected for gene expression analyses for this study. .....28

Table 2: Treatment preparation summary for IVM. ..................................................33

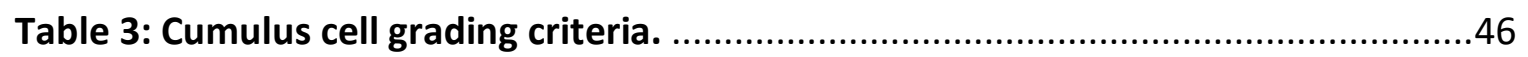

Table 4: Primers \& probes for genes selected for QPCR ..........................................56

Table 5: Optimised concentrations for primers \& probes for QPCR. ............................58

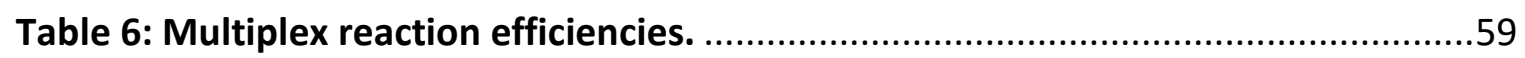

Table 7: Treatment preparation summary for granulosa cell proliferation assay. ........62

Table 8: Effect of OSF treatments on GVBD rate at 1 hour in IVM. Values represented

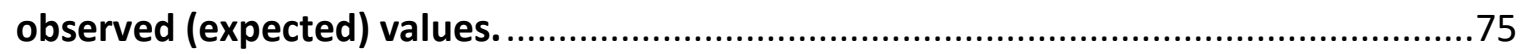

Table 9: Effect of treatments on GVBD rates at 7.5 hours in IVM. Values represented

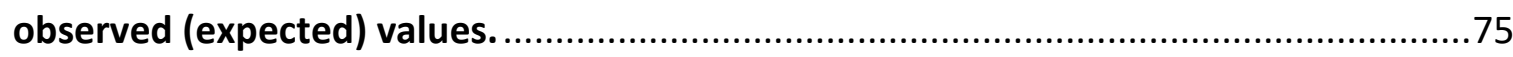

Table 10: Effect of treatments on GVBD rates at 24 hours in IVM. Values represented

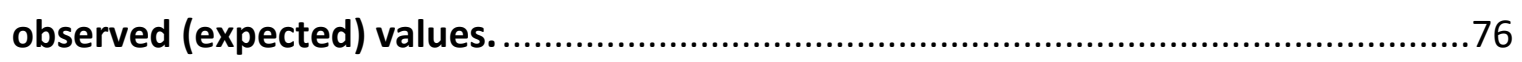

Table 11: Observing the effect of timepoints on GVBD rates. ..................................76

Table 12: Observing the effect of treatments on CC expansion at 1 hour in IVM.........77

Table 13: Observing the effect of treatments on CC expansion at 7.5 hours in IVM....77

Table 14: Observing the effect of treatments on CC expansion at 24 hours in IVM. .....78

Table 15: Observing the effect of timepoint on CC expansion......................................78 


\section{List of Abbreviations}

ART

BAX

BCL2

BMP

CAMP

CC

cGMP

CNP

$\mathrm{COC}$

$\mathrm{C}_{\mathrm{T}}$

CX

EGF

EGFR

FSH

FSHR

GC

GDF

GJ

GSH

GSSG

GST

GSTA1

GV

GVBD
Assisted Reproductive Technology

BCL2-Associated X

B-cell Lymphoma 2

Bone Morphogenetic Protein

Cyclic Adenosine Monophosphate

Cumulus Cell

Cyclic Guanosine Monophosphate

Natriuretic Peptide C-type

Cumulus Cell-Oocyte Complex

Cycle Threshold

Connexin

Epidermal Growth Factor

Epidermal Growth Factor Receptor

Follicle-Stimulating Hormone

Follicle-Stimulating Hormone Receptor

Granulosa Cell

Growth Differentiation Factor

Gap Junction

Reduced Glutathione

Oxidised Glutathione

Glutathione-S-Transferase

Glutathione-S-Transferase Alpha 1

Germinal Vesicle

Germinal Vesicle Breakdown 
hCG

HIF1A

IVC

IVF

IVM

IVP

$\mathrm{LH}$

LHR

MCB

MII

NPR

OSF

PDE

PR

PRDX6

ROS

SOD1

TGF

TNFAIP6
Human Chorionic Gonadotropin

Histone-Induced Factor 1 Alpha

In Vitro Culture

In Vitro Fertilisation

In Vitro Maturation

In Vitro Production

Luteinising Hormone

Luteinising Hormone Receptor

Monochlorobimane

Metaphase II

Natriuretic Peptide Receptor

Oocyte-Secreted Factor

Phosphodiesterase

Progesterone Receptor

Peroxiredoxin- 6

Reactive Oxidative Species

Superoxide Dismutase 1

Transforming Growth Factor

Tumour Necrotic Factor Alpha Induced Protein 6 




\section{CHAPTER 1: INTRODUCTION}

\subsection{In Vitro Production \& Oocyte Developmental Competency}

In vitro production (IVP) is a valuable biotechnological tool which utilises the combination of three key assisted reproductive technologies (ART); namely in vitro maturation (IVM), in vitro fertilisation (IVF) and in vitro culture (IVC). The goal of IVP is to produce embryos, from immature oocytes extracted from ovaries, in a culture dish. These technologies can be utilised to elucidate the mechanisms of early mammalian development with applications ranging from therapeutic treatment of human reproductive failure, preservation of gametes from animals with high genetic merit or conservation concerns, acceleration of breeding programs and efficiency of production, and can also be used to aid in the genetic improvement of livestock (Zhu et al., 2018).

The process of IVP in a clinical setting is still under refinement due to numerous insufficiencies with the technologies. For example, during in vivo follicular development, oocytes gradually undergo nuclear and cytoplasmic maturation that confer the oocyte with developmental competence. Oocyte developmental competency is the intrinsic measure of oocyte quality through its capacity to support the earliest stages of foetal life, embryo development and implantation. It is a key limiting factor in female fertility and ART (Hussein et al., 2006; Gilchrist et al., 2008). It is evident that in vivo produced embryos tend to be of better quality than those produced in vitro, which subsequently results in greater implantation, birth and survival rates. The timing and completion of oocyte maturation that results in a high quality, developmentally-competent oocyte is essential for successful embryo development. Therefore, it is perceived that IVM is a key step in providing quality oocytes for IVF and IVC, and the overall success of IVP (Luciano et al., 2018; Zhu et al., 2018)

\subsubsection{In Vitro Maturation}

The process of IVM refers to the extraction from the ovary of immature cumulus celloocyte complexes (COCs) for the purpose of maturing the oocyte in vitro. It has potential to serve as a beneficial clinical alternative and more cost-effective method to 
conventional IVF where expensive hormones must be administered (Edwards, 2007; Rose et al., 2014; De Vos et al., 2016). This technique was pioneered by Pincus and Enzmann (1935) who observed that the removal of COCs from immature follicles of the rabbit led to spontaneous nuclear maturation (i.e. resumption of meiosis) in the oocyte. Ongoing studies demonstrated that various mammalian oocytes (i.e. mice, sheep, pigs, cattle, nonhuman primates such as the rhesus monkey, and humans) could be matured in vitro (Edwards, 1965) and result in the successful birth of offspring (Goto et al., 1988; Sirard et al., 1988; Mattioli et al., 1989; Vanderhyden \& Armstrong, 1989; Cha et al., 1991; Cha et al., 1992; Yoshida et al., 1993; Trounson et al., 1994; Hasler et al., 1995; Keskintepe et al., 1995) Unfortunately in both clinical and agricultural settings, commercial IVM has proved to show significantly lower developmental competence and success rates, relative to conventional ART methods such as IVF on in vivo-matured oocytes. This is in part due to the inadequacies of in vitro environments in supporting complete oocyte maturation. While immature oocytes taken from antral follicles have usually reached nuclear maturation, cytoplasmic maturation only occurs in the last stages of the maturation process. Thus, if IVM methods fail to support the completion of cytoplasmic maturation prior to meiotic resumption, embryo development is compromised. One inadequacy that has been identified is the failure of the traditional IVM system in replicating the in vivo follicular environment (Buckett et al., 2007; Eppig et al., 2009; R. Li \& Albertini, 2013; Coticchio et al., 2015). As a result, the use of IVM as a method of ART has been largely restricted to research in domestic farm animals (De Vos et al., 2016). However in humans, it also provides a safer alternative for women at high risk of developing ovarian hyperstimulation syndrome (OHSS). OHSS is an often fatal syndrome that may occur due to the hormonal stimulation regime used to super-ovulate ovaries in conventional ART programmes, particularly those women with polycystic ovary syndrome (PCOS) (Edwards, 2007; Kovacs, 2007).

While it has been acknowledged that many factors affect developmental competence including but not limited to, oocyte origin (Lonergan et al., 1994), ovarian physiology (Urman et al., 1992; Hardy et al., 1995), follicle health (Blondin \& Sirard, 1995; Hagemann, 1999), and endocrine and paracrine signalling during follicular development (Blondin \& Sirard, 1995; Hagemann, 1999; Krisher, 2004; Sirard et al., 2006), the exact mechanisms 
that constitute developmental competency have not been fully elucidated. As such, identifying these key processes that influence this complex system may lead to the improvement of IVM systems and thus ultimately, improve ART success rates.

Recent studies have identified that the ovarian follicular environment is primarily mediated by the bi-directional intra-follicular communication between the oocyte and follicular somatic cells (Gilchrist et al., 2008). Oocyte-secreted growth factors (OSFs) play a pivotal role in inducing follicular development via complex paracrine signalling processes. Bone morphogenetic protein 15 (BMP15) and growth differentiation factor 9 (GDF9) are two closely-related, potent OSFs that are co-expressed during follicular development (Figure 1). These proteins are members of the transforming growth factorbeta (TGF- $\beta$ ) superfamily and have various biological functions. They act synergistically to influence granulosa cell (GC) differentiation into its distinct subtypes, promote cumulus cell (CC) expansion, survival, the attainment of oocyte developmental competency, ovulation rate, fertility and fecundity (Eppig et al., 1997; R. Li et al., 2000; McNatty et al., 2004; Hussein et al., 2005; Sugiura \& Eppig, 2005). Species-specific ratios of GDF9 to BMP15 are expressed in oocytes of mammals, which has previously been associated with ovulation rate and subsequently, litter size (Crawford \& McNatty, 2012).

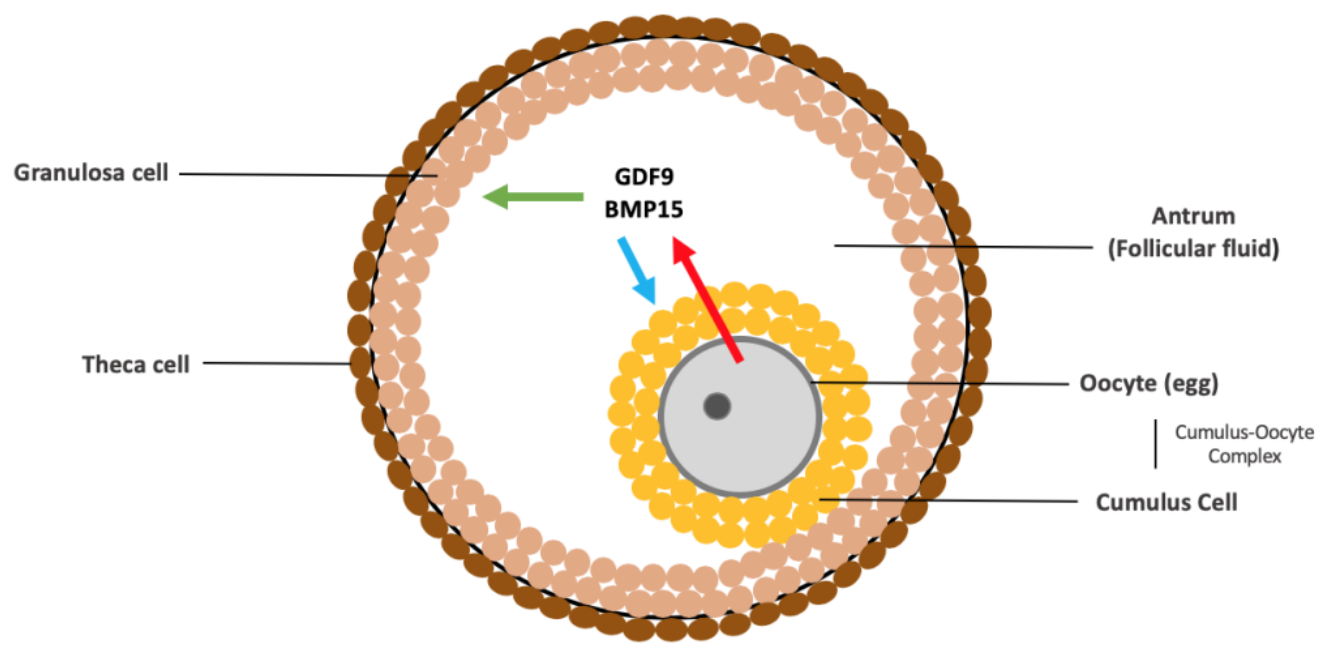

Figure 1: Depiction of bidirectional communication within the ovarian follicle. [RED arrow] Oocyte-secreted factors bone morphogenetic protein 15 (BMP15) and growth differentiation factor 9 (GDF9) are produced and secreted by the oocyte into the follicular fluid where they influence the proliferation and differentiation of granulosa cells (GC) [depicted by GREEN arrow] and promote cross-talk within the cumulus-oocyte complex (COC) to promote the attainment of developmental competence of the oocyte [depicted by BLUE arrow]. 


\subsection{Follicular Development in the Ewe}

During embryonic development, cells from the potent inner cell mass (ICM) undergo differentiation to form the various tissues of the foetus. A cohort of these potent ICM cells become germ cells which act as precursors to the gametes in males (i.e. spermatogonia) and females (i.e. oogonia). During gastrulation, these germ cells migrate from the yolk sac to the genital ridge and populate the developing gonads (i.e. bilateral ovaries or testes) (Driancourt, 1985; McNatty et al., 1995; Eckery, 1996; Lundy, 1999). During the first trimester of gestation, germ cells within the developing gonads of females differentiate into oogonia and divide mitotically, peaking at approximately a population of 900,000 germ cells around 75 days into gestation (B. H. Erickson, 1966; McNatty et al., 1995; Eckery, 1996). The vast increase in numbers of dividing oogonia attract cells from the ovarian surface epithelium to surround the oogonia as pre-granulosa cells. These dividing oogonia and associated pre-granulosa cells are isolated from the ovarian stroma through the formation of ovigerous cords or nests. By the second trimester, around $80 \%$ of oogonia undergo atresia, while the remainder enter meiosis and arrest at prophase I to become oocytes (Zamboni et al., 1979; Sawyer et al., 2002). Around the time of birth, each meiotically-arrested oocyte surrounded by a single flattened layer of GCs bud off the basal end of the ovigerous cords to form primordial follicles (Juengel et al., 2002; Sawyer et al., 2002).

From the time of birth, primordial follicles leave the pool of non-growing follicles one at a time in a sequential manner. The first primordial follicles to form are the first to initiate growth (Figure 2) (Sawyer et al., 2002). The transition from a primordial follicle to a growing follicle is marked by the morphological differentiation of the GCs from a squamous to cuboidal shape, and is termed a primary follicle (Lintern-Moore \& Moore, 1979). The proliferation of the GC which results in numerous layers surrounding the oocyte is characteristic of a secondary follicle. Up until the time that proliferation of GCs eventuates into the formation of a small fluid-filled cavity known as the antrum, follicles may be grouped as preantral follicles. The growth of follicles from the primordial to secondary stage of development is regulated by ovarian factors, and are not influenced by the reproductive hormones from the brain, in particular the gonadotrophins. 
It is only following puberty (i.e. 5-12 months in sheep depending on breed, body weight, nutrition and season of birth; (McNatty et al., 1995) that follicles progress pass the preantral stage. Antral or tertiary follicles consist of an antrum that contains follicular fluid, a complex mixture of substrates and factors from the circulatory system, and the follicular cells including the oocyte. The appearance of an antrum divides the GCs into two distinct phenotypes; the mural GC and the cumulus cell (CC) (Eppig et al., 1997; R. Li et al., 2000; Diaz et al., 2007). The mural GCs located in the periphery of the follicle actively regulate steroidogenesis while the CCs, which are in direct proximity to the oocyte, are involved in metabolic nurturing of the oocyte. However, follicular fluid composition tends to show major deviations across species in terms of amino acid and energy substrate concentrations and are likely dependent on follicle size and the metabolic requirements of the follicle (Sinclair et al., 2008).

The formation of the antrum also marks the transition from a gonadotrophin-independent follicle to a gonadotrophin-dependent follicle (Zamboni, 1974; Hirshfield, 1991; Rodgers \& Irving-Rodgers, 2010; Hummitzsch et al., 2013). The gonadotrophins, namely luteinising hormone ( $\mathrm{LH})$ and follicle-stimulating hormone (FSH), are secreted by gonadotroph cells in the anterior pituitary gland upon stimulation by the hypothalamic-derived peptide gonadotropin-releasing hormone $(\mathrm{GnRH})$. The actions exerted by FSH to facilitate follicular growth past the preantral stage has been well characterised (G. F. Erickson et al., 1979; El-Hayek et al., 2014). The binding of FSH to their receptors (i.e. FSHR; folliclestimulating hormone receptor) on GCs play an important role in the acquisition of oocyte developmental competency, regulation of meiosis and ovulation.

In turn, the secretion of gonadotropins from the pituitary gland is regulated via negative and positive feedback from the follicles in the form of steroid (oestradiol, progesterone and testosterone) and peptide (activin, inhibin and follistatin) hormones. The acquisition of $\mathrm{LH}$ receptors on the GCs mark the follicle as destined to ovulate (i.e. pre-ovulatory) and enables the follicle to synthesise and secrete large amounts of oestradiol. The rising oestradiol levels from the large pre-ovulatory follicle eventually triggers the pre-ovulatory LH surge. Up until this time, the meiotically-arrested oocyte contains an intact germinal vesicle (GV). The pre-ovulatory LH surge causes meiosis to resume and the final stages of 
nuclear and cytoplasmic maturation in the oocyte are completed (Tsafriri et al., 1972; Eppig, 1980).

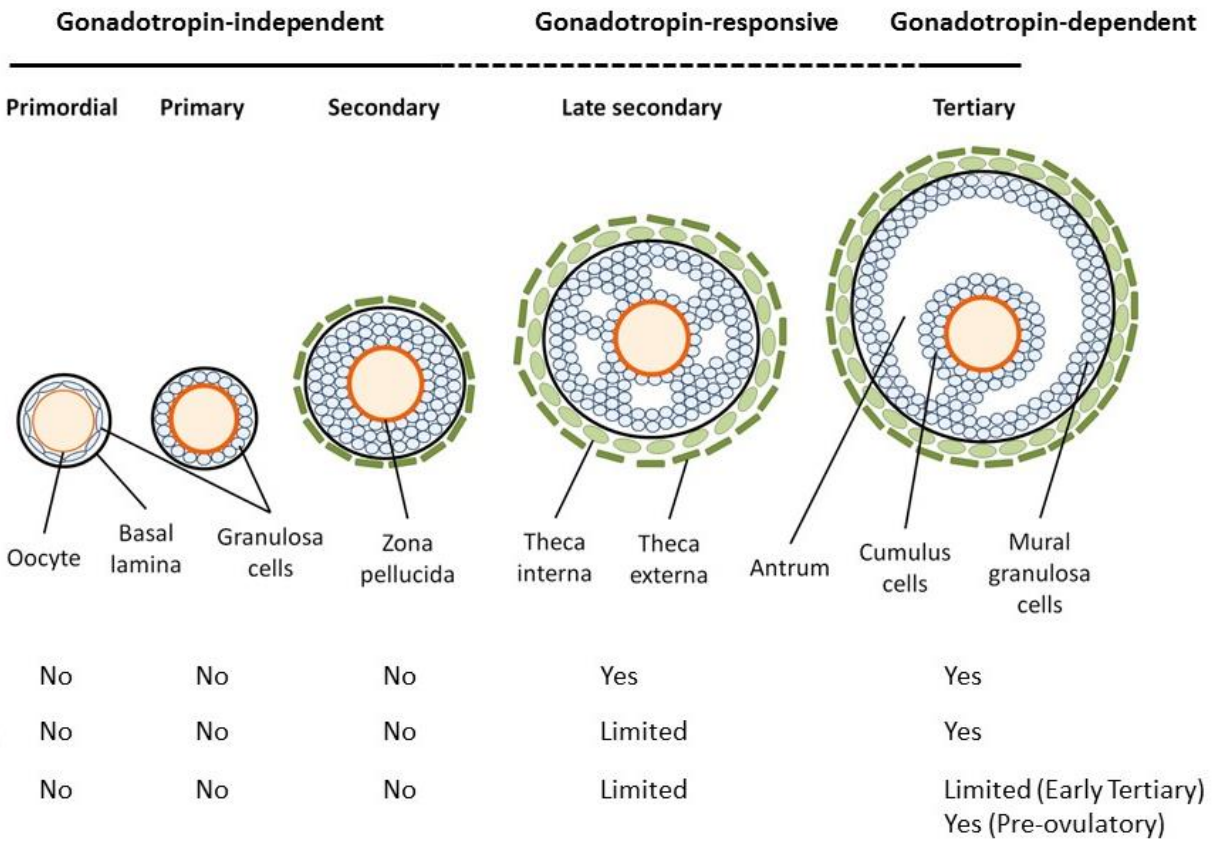

Figure 2: Follicular development from a primordial to tertiary stage. The gonadotropindependent, gonadotropin-responsive and gonadotropin-independent stages of development are depicted above. Stages in which nuclear maturation, cytoplasmic maturation and developmental competency are acquired has also been indicated. Diagram supplied and used with permission by Clark (2018).

\subsection{Oestrous Cycles in the Ewe}

The oestrous cycle represents the cyclical pattern of ovarian activity (Forde et al., 2011). Ewes are seasonally polyoestrous mammals, with oestrous cycles lasting an average of 1617 days. The cycles consist of a 5-7 day follicular phase in which follicular maturation and ovulation occurs. This is followed by a 10-14 day luteal phase which is characterised by the formation of the corpus luteum. The oestrous cycle continues until pregnancy is achieved or until the ewe returns to an anoestrus state at the end of the breeding season (Legan \& Karsch, 1979; Bartlewski et al., 2011).

In ewes, the development of antral follicles occurs in waves (non-ovulatory and ovulatory) during the ovarian cycle which is reflective of the levels of FSH secretory patterns. The exact number of waves has been heavily debated among many researchers, with the 
consensus being two or three. This cyclical activity is heavily influenced by many factors including sheep breed (Holtz \& Lindloff, 1976; Scaramuzzi \& Radford, 1983; Hari et al., 1988; Sirois \& Fortune, 1988; Souza et al., 1997; Ginther et al., 2003; Bracken et al., 2007; Baby \& Bartlewski, 2011; Bartlewski et al., 2011; Forde et al., 2011).

Most sheep are considered short-day breeders, with the breeding season beginning as daylight becomes more scarce (autumn and winter). Sheep appear to have developed an evolutionary mechanism during periods of long daylight which cause ewes to become subfertile. During this time, commonly referred to as seasonal anoestrous, ovaries are absent of growing follicles and corpus lutea and as a result, embryo survival rates are low. This is due to sheep being extremely photoreceptive, with the levels of light exposure directly influencing the regulation of critical precursors and hormones which are essential in breeding. Melatonin, well known as "the hormone of the dark", is a key regulator in the maintenance of body homeostasis and circadian rhythms. It is produced at greater levels by the brain during periods of low daylight, and has a critical role in the positive feedback of $\mathrm{GnRH}$ production and the rest of the hypothalamic-pituitary-gonadal axis (Misztal et al., 2002). Interestingly, rams are also seasonal breeders with decreased sperm production, increased abnormal spermatozoa and decreased fertility during the photoperiod (Schanbacher, 1978; Milczewski et al., 2015; Belkadi et al., 2017).

\subsection{Maturation \& the Acquisition of Developmental Competency}

Oocyte maturation is a lengthy and crucial process in which oocytes acquire the intrinsic ability to support the subsequent stages of development, ultimately reaching embryonic genome activation. This involves the complex events of nuclear and cytoplasmic maturation which are two developmentally distinct, but synchronised, processes. Both processes must occur for an oocyte to mature successfully.

\subsubsection{Nuclear Maturation}

Meiotic resumption and the acquisition of oocyte developmental competency only occurs in the final stages of follicular development. While nuclear maturation is normally acquired at the time of antral formation in a tertiary follicle (Figure 2), full developmental 
competency of the oocyte can only be attained following the preovulatory LH surge (Park et al., 2004; Mehlmann, 2005). Up until this time, the maintenance of meiotic arrest is crucial in enabling the oocyte to attain full developmental competency. This process relies on the coordinated exchange of appropriate nutrients, energy substrates, metabolites, second messengers, ions and paracrine factors between the oocyte and follicular somatic cells. This bidirectional cross-talk between cells is maintained by gap junctions (GJs), small open-ended protein channels comprised of connexins that are physically coupled to adjacent cells (Gilula et al., 1978; Wiesen \& Midgley, 1993; Simon et al., 1997).

Meiotic arrest at prophase I (germinal vesicle oocyte; GV), is achieved through a constant supply of cyclic nucleotides (cyclic adenosine monophosphate; CAMP and cyclic guanosine monophosphate; $c G M P$ ). Whilst some cAMP is independently synthesised in the oocyte by adenylyl cyclase (AC) and the activation of G-protein coupled receptor (GPCR), a substantial source of intra-oocyte CAMP and CGMP is provided via its surrounding somatic cells (Figure 3) (Mehlmann, 2005). The intra-oocyte levels of these cyclic nucleotides are maintained by degradation through phosphodiesterases (PDEs) and stimulation via mediators such as C-type natriuretic peptide (CNP), a paracrine factor produced by GC (Tsafriri et al., 1976; M. Zhang et al., 2010).

The binding of follicle-stimulating hormone (FSH) to its receptor (FSHR) on the GC results in the upregulation of the natriuretic peptide $\mathrm{c}$ precursor gene (NPPC) and the production of oestradiol. The NPPC protein is cleaved into its 22-amino acid derivative CNP, while oestradiol results in the upregulation of natriuretic peptide receptor 2 (NPR2) on the CC. As CNP is released from the GC, it binds to and activates NPR2. This activation results in the synthesis of CGMP within the CC and is transferred to the oocyte via GJs. The GJ protein connexin 43 (Cx43) physically couples CCs and GCs, whilst the innermost CCs (corona radiata cells; CR), synthesise the GJ protein connexin 37 (Cx37) which physically couples the $\mathrm{CC}$ to the oocyte membrane. The entrance of cGMP maintains CAMP levels by inhibiting its hydrolysis by PDEs, in particular oocyte specific PDE3A (Reinhardt et al., 1995; Tsafriri et al., 1996). Elevated cAMP levels in the oocyte sustains meiotic arrest by preventing the GV breakdown (GVBD) through the phosphorylation of cyclin-dependent kinase 1 (CDK1) and inactivation of maturation-promoting factor (MPF) (Cho et al., 1974; M. J. Zhang et al., 2009; Tripathi et al., 2010). 
As follicular growth progresses through a cross-talk between OSFs and FSH regulation, the follicle most responsive to FSH dominates and acquires LH receptors (LHR) on their GCS (i.e. the pre-ovulatory follicle) (Webb \& England, 1982). The acquisition of the LHR on GCs also plays an important role in the resumption of meiosis. The oocyte and CC do not display such receptors therefore, the GC must transduce this signal to the oocyte. This is achieved through epidermal growth factor (EGF)-like ligands such as amphiregulin, betacellulin and epiregulin, which are synthesised and secreted by the GC in response to FSH (Caixeta et al., 2013; Richani et al., 2013; L. Yang et al., 2016). These EGF-like ligands bind to functional EGF receptors (EGFRs) on the CC, which initiates a mitogen-activated protein kinase $3 / 1$ (MAPK3/1) signalling cascade that results in the decreased expression of NPR2. (Amsterdam et al., 1975; van Tol et al., 1996; Park et al., 2004). The expression levels of EGFR on CCs and oocytes increases throughout follicular development. GDF9 and BMP15 are key players together with CAMP and EGFs in the resumption of meiosis through their ability to promote functionality of the EGFRs (Sugimura et al., 2015). An influx of LH initiates an ovulatory cascade and as a result, triggers the secretion of hyaluronic acid by the CC. This induces CC expansion and mucification, luteinisation of GCs and the phosphorylation and closure of GJ networks throughout the follicle to prevent further communication between the CC and oocyte. The closure of GJs immediately reduces the supply of cyclic nucleotides into the oocyte and results in the hydrolysis of CAMP by PDE3A. The loss of intra-oocyte cAMP levels results in meiotic resumption where progression to metaphase II (MII) is characterised by GVBD in the oocyte and extrusion of the first polar body. 


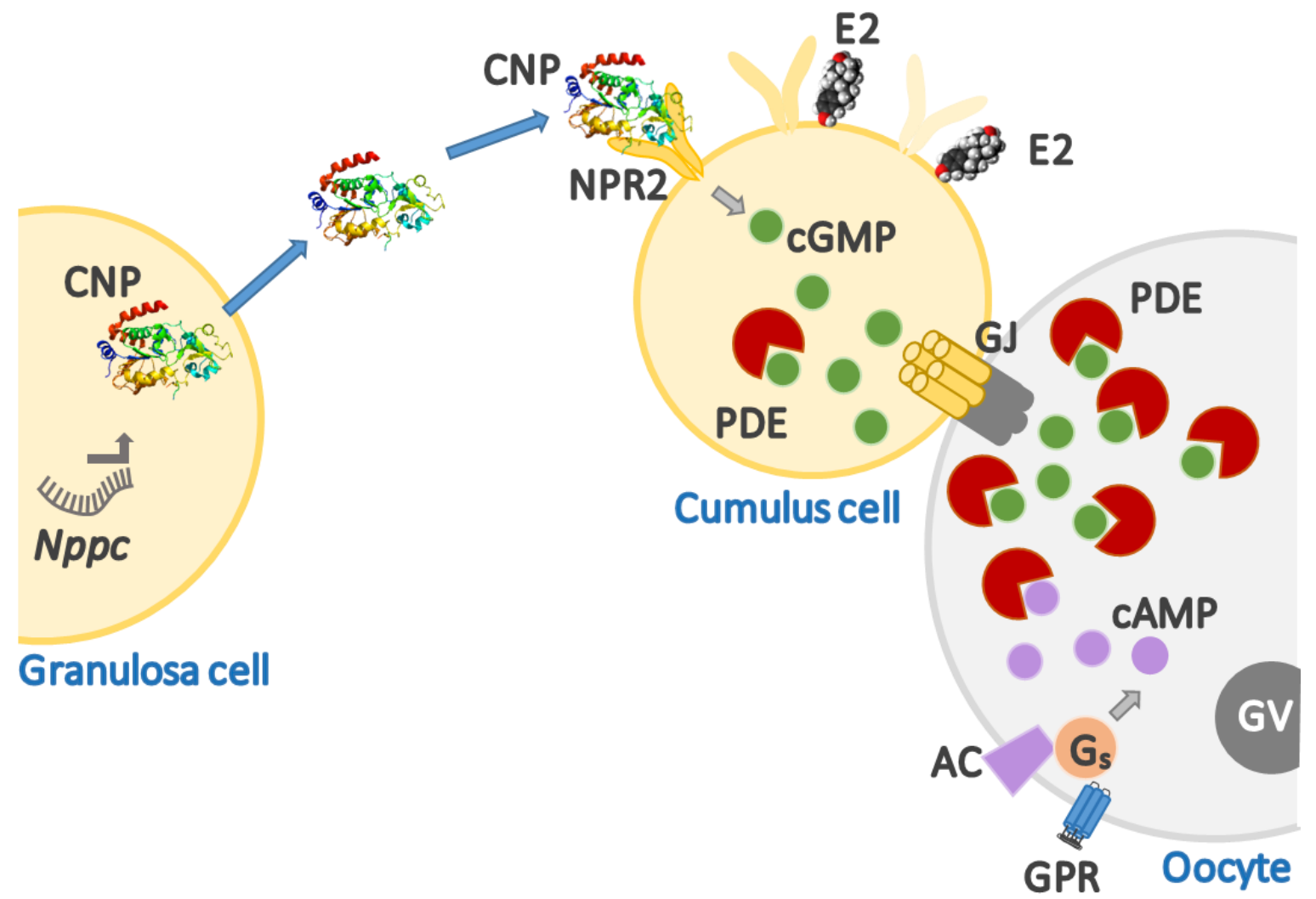

Figure 3: The molecular overview of maintaining meiotic arrest and nuclear maturation within the mammalian COC. The binding of follicle-stimulating hormone (FSH) to its receptor (FSHR) on the granulosa cell (GC) results in the production of oestradiol (E2). E2 results in the upregulation of natriuretic peptide receptor (NPR). C-type Natriuretic Peptide (CNP) is produced and secreted by the GC and binds to NPR on the cumulus cell (CC). The activation of NPR results in the synthesis of cyclic guanosine monophosphate (cGMP) within the CC. cGMP is transferred via gap junctions (GJs) to the oocyte where is reduces the hydrolysis of cyclic adenosine monophosphate (CAMP) by inhibiting phosphodiesterases (PDEs) that normally hydrolyse and reduce cAMP levels. This results in the maintenance of elevated CAMP levels within the oocyte and ultimately sustains meiotic arrest by preventing germinal vesicle breakdown (GVBD). CAMP is produced intrinsically by the oocyte via G-protein coupled receptors (GPCR) and adenylyl cyclase and in part, by the CC and transferred to the oocyte via GJs. Figure provided with permission for use by Dr. Janet Pitman.

\subsubsection{Cytoplasmic Maturation}

The role of specific organelles, nuclear transcripts and proteins in the cytoplasm of the oocyte (i.e. ooplasm) are fundamental to the overall process of cytoplasmic maturation however, their transit are highly restricted within the $\mathrm{COC}$ which is limited to paracrine signalling and the direct transfer of small molecules $(<1 \mathrm{kDa})$ via GJs (Flagg-Newton et al., 1979; Bruzzone et al., 1996). In the mammalian oogenesis model, oocytes from preantral 
follicles enter a growth phase in which they accumulate vast amounts of maternal mRNA and proteins that are critical for the progression of preimplantation embryonic development to the 8-cell cleavage stage in cattle and sheep (Crosby et al., 1988; L. Li et al., 2013; Macaulay et al., 2014). The transcription and storage of maternal mRNA occurs during follicular growth where they are packaged and stored as stable, transiently-inactive ribonucleoprotein particles to protect them from nucleolytic degradation. Upon GVBD and meiotic resumption, chromatin condenses and the oocyte becomes transcriptionallyquiescent and dependent on these stores until embryonic genome activation (EGA) occurs. Thereafter, the gradual synthesis of new proteins and genes contributes to the on-going success of implantation and development. Thus through the process of polyadenylation and deadenylation, these stored mRNAs and proteins are rapidly degraded during cytoplasmic maturation of the oocyte and the early cleavage stages of the embryo. Polyadenylation of mRNA, defined as the addition of adenine to the $3^{\prime}$ terminus of mRNA to form a poly-(A)-3' tail, occurs in the nucleus of the oocyte, and is transported to the ooplasm to initiate the translation of proteins and deadenylation (i.e. the removal of adenine) which simultaneously results in the degradation of particular mRNAs.

While it has been traditionally perceived that these mRNAs and proteins are synthesised intrinsically by the oocyte, recent reports of large particles such as proteins, microbes and organelles or organelle-like structures within the ooplasm suggest that alternative cellular extensions are potentially involved in cellular exchange within the COC (Macaulay et al., 2014). The corona radiata cells project close-ended intra-oocyte extensions, called transzonal projections that directly penetrate the zona pellucida and contact the oocyte membrane. While the function of transzonal projections in intracellular communication continues to be elucidated, evidence suggests that they may be involved in the transfer of mRNAs, which are too large to be exchanged via GJs, from the corona radiata to the oocyte. These transported mRNAs may assist in the maintenance of transcriptional suppression of the oocyte during maturation (Macaulay et al., 2014).

Cytoplasmic maturation also involves the trafficking of cytoplasmic organelles, which increase in abundance and redistribute themselves within the ooplasm. Cytoskeletal filaments are dynamic and adaptable as they undergo modifications according to the 
needs of the cell. This system plays a major role in chromosome segregation and partitioning during cell division, as well as the trafficking of molecules and organelles into cells. The three main filaments types of the cytoskeleton are (i) microtubules, (ii) actin and (iii) intermediate filaments, each of which consists of distinct subunits that are characteristic to each type. All three polymers are maintained by weak, noncovalent interactions that allow rapid association and dissociation without the need for forming or breaking covalent bonds. Among these three filament types, microtubules are the most involved in organelle reorganisation within the oocyte (Ferreira et al., 2009). The microtubule subunits adhere to motor proteins (i.e. kinesin, dynein and dynactin) which bind to organelle membranes and promote transport along the microtubule.

The repositioning and morphological changes of organelles in the ooplasm are dependent on the needs of the oocyte during different stages of maturation. Changes in the abundance and redistribution of the endoplasmic reticulum, Golgi apparatus, cortical granules, ribosomes, vesicles and mitochondria have been reported (Ferreira et al., 2009). Mitochondria are the primary component of the metabolic machinery responsible for meeting the energy demands of the cell. Protein synthesis and phosphorylation are key processes in maturation and consume high levels of energy in the form of adenosine triphosphate (ATP). Over the course of oogenesis, mitochondria significantly increase in numbers and position themselves in areas of high energy consumption during critical periods of the cell cycle (Ferreira et al., 2009). For example, the human oogonia contains 300 mitochondria, the primordial oocyte 6000 mitochondria, and the mature oocyte 300,000-400,000 mitochondria (Jansen \& de Boer, 1998). Interestingly, it has been suggested that this increase in number is a result of maturation but also due to potential trafficking into the oocyte from surrounding somatic cells (Ferreira et al., 2009).

Major controversies still remain in regards to the relationship of mitochondrial distribution and ATP on the acquisition of developmental competency. Some studies have found discrepancies between the distribution of mitochondria in in vivo versus in vitro settings. This has been implicated as a factor which may contribute to the asynchronous maturation issues with IVM (Ferreira et al., 2009). Moreover, murine IVM models have demonstrated that early embryos ( 72 hours in culture) with lower ATP levels in their cytoplasm have slower development. It is important to note that slow development 
during this period is not necessarily abnormal and is likely a defence mechanism adapted against reactive oxidative species (ROS). These toxic metabolites produced by mitochondrial metabolism can have detrimental effects on development (Ferreira et al., 2009). Studies have previously suggested that if mitochondrial activity was high prior to EGA, embryo development would persist more rapidly but an excessive build-up of ROS would ultimately reduce chances of embryo survival. This can be deemed as the "quiet embryo hypothesis" (Leese, 2002). In opposition to this theory, Dumollard et al. (2006; 2007) suggested that this low mitochondrial activity in the oocyte is more due to the fact that these mitochondria tend to be immature and inactive, which would ultimately minimise ROS production. Moreover, the gradual increase of mitochondrial activity throughout development is crucial during transcriptional quiescence in the transition from a mature oocyte to the preimplantation embryo, prior to EGA. In addition, metabolites, particularly antioxidants which increase during maturation, play a crucial role in neutralising ROS throughout crucial stages of development (C. M. H. Combelles et al., 2009). This is expanded upon in Section 1.4.3.

Both nuclear and cytoplasmic maturation must be achieved for the successful completion of oocyte maturation and ultimately, the acquisition of developmental competency. Unfortunately, the removal of the COC from its physiological environment prior to acquirement of developmental competency results in spontaneous nuclear maturation (meiotic resumption) prior to the completion of cytoplasmic maturation. This is because the $\mathrm{COC}$ has been removed from the follicular environment containing meiotic inhibiting factors which are not maintained in vitro. Traditionally, IVM media was supplemented with pharmacological agents that stimulated CAMP production, such as forskolin, or that reduced cAMP catabolism such as 3-isobutyl-1-methylxanthine, a broad spectrum PDE inhibitor. These were added to delay meiotic resumption in an attempt to allow the cytoplasm to undergo its final maturational stages. Recently, refinements have been made to IVM systems in order to sustain meiotic arrest with "pre-IVM" maturation steps. This involves the addition of CNP to mimic events that occur within the ovarian follicle making it a more physiologically-relevant system (Albuz et al., 2010; Yamashita et al., 2011; Richani et al., 2014; Santiquet et al., 2017). This has shown to substantially improve oocyte developmental competency rates as it allows additional time for the oocyte to 
complete the maturational process (i.e. complete appropriate cytoplasmic maturation) prior to meiotic resumption.

\subsubsection{Metabolic Influence in Follicular Maturation}

The dynamic processes which occur during nuclear and cytoplasmic maturation in the COC require energy. The cells in the ovarian follicle, especially the COC, exploit a variety of metabolic substrates in the form of carbohydrates, amino acids, and lipids to support their energetic need throughout oogenesis. The overall ratio of these metabolites throughout different phases of maturation can act as insightful biomarkers for developmental competency.

Carbohydrates, particularly glycolytic metabolites and substrates, play a crucial role in early development. Studies by Sutton et al. (2003) have demonstrated that in in vitro culture systems, mature COCs tend to be more metabolically demanding, consuming twofold more glucose, oxygen and pyruvate, relative to an immature COC. The importance of the bidirectional paradigm of the $\mathrm{COC}$ is crucial to consider in terms of glycolytic metabolism, as the oocyte itself has a low capacity to utilise glucose (Biggers et al., 1967; Donahue \& Stern, 1968; P. D. Cetica et al., 1999; P. Cetica et al., 2002). In the COC, the CC is the primary site for glycolytic metabolism and through GJs, is able to provide the oocyte with essential intermediate metabolites and substrates which drive the progression of critical maturational processes and regulation of the attainment of development competency.

Glucose is metabolised through four different pathways in the COC (Sutton-McDowall et al., 2010). The majority is metabolised via glycolysis, an enzymatic process for converting one molecule of glucose into two molecules of pyruvate in a step-wise fashion. In anaerobic conditions, pyruvate is converted into lactate. Conversely in aerobic conditions, pyruvate is broken down further and converted into acetyl-coenzyme A (acetyl CoA) which enters the Krebs Cycle (also known as the tricarboxylic acid cycle and citric acid cycle) for the production of ATP and NADH. Products of the Krebs Cycle are transported via GJs to the oocyte which is a major site for oxidative metabolism. They are used as substrates for oxidative phosphorylation at the mitochondria yielding large amounts of ATP. However, increased mitochondrial activity results in excessive ROS production which 
can have detrimental effects on cell development. Oxidative stress has been associated with various negative implications on both the oocyte and embryo (Dumollard et al., 2007). It can induce apoptosis resulting in fragmented cells (Johnson \& Nasr-Esfahani, 1994; H. W. Yang et al., 1998), maternal and postovulatory aging (Tarin, 1996; Fissore et al., 2002).

The pentose-phosphate pathway (PPP) serves as an alternative route for glucose metabolism (Reviwed by Sutton-McDowall et al., 2010). This pathway has non-oxidative and oxidative stages. Glucose is able to enter at either of these stages. The non-oxidative arm plays an important role in the synthesis of purines which are essential to nuclear maturation. The oxidative arm has important implications in the redox state and maintenance of cytoplasmic integrity of the oocyte through the production of antioxidants (i.e. glutathione). The oxidative PPP pathway utilises glucose by producing NADPH which reduces oxidised glutathione (GSSG) into reduced glutathione (GSH), a glutamate-cysteine-glycine tripeptide. This antioxidant in its reduced form has been positively attributed to oocyte developmental competency (Jiao et al., 2013), reduced polyspermy (Urner \& Sakkas, 1999) and improved embryo quality (Jiao et al., 2013). However, only a small proportion of glucose $(\sim<3 \%)$ is utilised by this pathway in the bovine COC (Urner \& Sakkas, 1999).

The third pathway which can utilise glucose in the $\mathrm{COC}$ is the hexosamine biosynthesis pathway (HBP). This is a fuel-sensing pathway that is responsible for the generation of substrates for the extracellular matrix (Reviwed by Sutton-McDowall et al., 2010). Here, glucose is utilised for the production of hyaluronic acid for CC expansion at the final stages for oocyte maturation. Expansion and mucification of the $\mathrm{CC}$ is crucial in an in vivo situation, to ensure preparation for ovulation and implantation of the oocyte into the infundibulum. In addition, an early step in the pathway can lead to the O-linked glycosylation of proteins. O-linked glycosylation can have deleterious effects on oocyte health as these resultant proteins can alter protein signalling pathways and subsequently, the activation of downstream targets (Frank et al., 2014).

The polyol pathway is the least characterised of these glucose utilisation pathways used by the COC (Reviwed by Sutton-McDowall et al., 2010). This pathways oxidises glucose to sorbitol and fructose. Glucose utilisation by this pathway can increase significantly in 
hyperglycaemic conditions. In cases of prolonged hyperglycaemic conditions, an intracytoplasmic accumulation of sorbitol and fructose is believed to be implicated in diabetic pathology as both of these metabolites have poor membrane permeability. This can result in reduced NADPH production, inducing hypoxia and altering the ratios of lactate and pyruvate within the cell. However, it is important to note that sorbitol and fructose are not completely deleterious on the COC. It is believed that the PPP pathway and these metabolites at a homeostatic level are involved in ovarian tissue regulation and remodelling during the oestrous cycle (Sutton-McDowall et al., 2010)

Amino acids act as precursors for the synthesis of proteins, nucleotides and various signalling molecules that play a crucial role in both oogenesis and embryo development. Different amino acids serve as energy substrates for various processes in the cell including but not limited to the regulation of $\mathrm{pH}$, osmolarity, heavy metal chelators, and methyl group donors (Gu et al., 2015). The reproductive tract, and particularly the follicular fluid, contain many different amino acids. The high concentrations of glutamine, glycine and alanine play key roles in multiple different components throughout oogenesis. The growing oocyte has high RNA and protein synthesising activity with which glutamine and glycine, as well as aspartate and methionine are required for de novo synthesis of purines and pyrimidines for nucleotides of mRNA. Glutamine and glycine are also required by the TCA cycle and oxidative phosphorylation in order for the oocyte to meet its ATP demands. Glycine, glutamate and cysteine are also key precursors for the formation of GSH.

Most studies investigating the effects of amino acids in development have been carried out in vitro, with great focus on the embryo over the oocyte. In vitro studies have found that the embryo has a large capacity for the consumption of glutamate and arginine, while producing alanine, during preimplantation developmental stages. This appears to be consistent across multiple species although the utilisation of other amino acids is dependent upon the culture conditions, developmental stage of the embryo and the species (Gu et al., 2015). The addition of arginine and aspartate in culture media has been reported to have beneficial effects on embryonic development, while the addition of glutamine and aspartate has reduced polyspermy rates (Gu et al., 2015). The addition of amino acids into IVM media can also enhance oocyte quality and the attainment of developmental competency. The addition of glutamine has been shown to promote 
nuclear maturation ( $G u$ et al., 2015). Glutamine also initiates signalling cascades in the HBP pathway to induce the production of hyaluronic acid from glucose utilisation for CC expansion (Reviwed by Sutton-McDowall et al., 2010). The addition of cysteine to IVM media assists in protein folding and the production of pyruvate.

Lipids serve as an important endogenous energy source and building block for growth and development in the oocyte. Most lipids in the ooplasm are synthesised and stored longterm within specialised organelles deemed as lipid droplets. These organelles store neutral lipids, usually in the form of triacylglycerides and sterol esters within a hydrophobic phospholipid monolayer studded with various different proteins. Lipid droplets are primarily accumulated during cytoplasmic maturation. Stored lipids can serve as substrates for the biosynthesis of steroid hormones, prostaglandins and membranes as well as second messengers in cell signalling. The synthesis of membranes is especially important during rapid cell divisions during cleavage in the zygote. However, lipid-derived fatty acids are a very important energy source as they can be utilised by the mitochondria to produce acetyl-CoA via $\beta$-oxidation. This pathway yields more ATP from a single lipid than the oxidative phosphorylation of a glucose molecule (Dunning et al., 2014). Experiments by Dunning et al. (2010) that inhibited $\beta$-oxidation during IVM and early embryo development showed a significant perturbation in oxygen consumption and blastocyst development in mice.

Despite the importance of lipids during maturation, supra-optimal lipid accumulation within the cytoplasm can compromise developmental competency and foetal outcome, as well as impair cryopreservation (Abe et al., 1999). Cryopreservation is important for IVM as some clinics will not transfer fresh embryos resulting from IVM due to reduced live birth rates (Walls et al., 2015). This also highlights how poor maternal health, such as obesity, can heavily influence infertility.

However, it has been suggested that perhaps lipid droplet accumulation and their interactions in the cell should be considered by species (Dunning et al., 2014). Some animals, such as pigs and some ruminants, have a high lipid droplet distribution within the ooplasm which is positively associated with developmental competence. In contrast, high intracellular lipid levels may also be associated with low quality oocytes and fertility failure in humans (Jungheim et al., 2011). It could be implied that this could be due to litter size 
where poly-ovulatory animals, such as the pig, require a large lipid reservoir and energy in order to support multiple embryos until the placenta can take over. This level of energy could be detrimental to both oocytes and embryos of mono-ovulatory species due to the production of ROS and reduced cryo-tolerance.

\subsubsection{Ovulation \& Developmental Competency}

Following the pre-ovulatory $\mathrm{LH}$ surge and completion of oocyte maturation, the COC ruptures from the ovarian follicle and is captured by the infundibulum, where it awaits insemination and fertilisation (Sugimura et al., 2017). The resultant luteinised debris of GCs and theca cells restructure to form a corpus luteum (CL). The completion of oocyte developmental competency is also acquired around this stage.

In a clinical setting, IVM oocytes show reduced developmental competency than those that have matured naturally within an in vivo environment. Consequently, the demand for methods to assess developmental competency in oocytes is increasing as embryo grading systems alone fail to improve ART success rates (Dyer et al., 2016). There is considerable interest in identifying biomarkers of developmental competency. While biomarkers within the oocyte are an obvious choice, testing for developmental competency of oocytes in a non-invasive manner, without compromising its chances for fertilisation, embryo development and implantation, is an area of ongoing research (Sinclair et al., 2008; Rawe \& Combelles, 2009). Therefore, development of competency biomarkers for materials that are routinely discarded during ART prior to fertilisation serve as attractive alternative to the oocyte.

Due to the bidirectional communication that occurs throughout development between the oocyte and CC, and their juxtaposed positioning, the CCs are included in the group of materials discarded during ART procedures and have been utilised to investigate for biomarkers of oocyte developmental competency. There are clear benefits of profiling maternally-derived mRNA and proteins as they are key during early embryonic development and their expression levels within the COC, making them ideal biomarkers with potential prognostic value in terms of oocyte quality (Rawe \& Combelles, 2009). Whilst there are two somatic cell populations within the follicular cell membrane (GCs and CCs), the CCs are believed to be a better candidate for biomarkers due to their close 
association with the oocyte throughout maturation. The CCs are physically coupled to the oocyte via the GJ protein Cx37, which is fundamental in the process of exchanging materials that contribute to the attainment of complete maturation and developmental competency. The removal of the CCs from the COC during early stages of maturation has previously demonstrated to have detrimental effects on the development of the oocyte (Virant-Klun et al., 2013; Uhde et al., 2018). The expression of GJ genes such as CX37 and CX43 are therefore both important developmental competency markers as they are associated with the transport of crucial molecules and paracrine factors that improve oocyte quality.

There are several genes in CC with various functions that have been well reported in literature and associated with development. In human CCs, expression of genes such as prostaglandin-endoperoxide synthase 2 (PTGS2), hyaluronan synthase 2 (HAS2), gremlin 1 (GREM1), and steroidogenic acute regulatory protein (STAR) have been positively correlated with high embryo quality and blastocyst development while genes such as Bcell lymphoma 2 (BCL2), phosphoenolpyruvate carboxykinase 1 (PCK1), versican (VCAN), PTGS2, GREM1 and phosphofructokinase (PFKP) have been associated with pregnancy (Bunel et al., 2014). The CC can also indicate negative outcomes in embryo quality or pregnancy outcome from the inappropriate timing or altered expression of genes such as progesterone receptor (PR), CX43/GJA1, amphiregulin (AREG), coenzymes (SCD1 \& SDC5), glutathione peroxidase 3 (GPX3), heat shock protein B1 (HSPB1) (Bunel et al., 2014). While there are many candidates with distinct functions, many commonly studied CC genes reported in literature that have been associated with developmental competency and maturation are key regulators of expansion such as HAS2, VCAN, TNF-alpha-induced protein 6 (TNFAIP6), PTGS2 and GREM1 (Hsieh et al., 2009). However, experiments conducted by the Pitman laboratory (Victoria University of Wellington, NZ) in the past have found that expansion genes are only good indicators of oocyte quality in vivo (Murray, 2020). The process of mucification and expansion of CC are crucial as they allow the capture of the oocyte by the infundibulum (Sugimura et al., 2017). It is important to note that while expansion is a good indicator of maturation and positively correlated with developmental competency in vivo, it is not necessarily a biomarker of developmental competency in an in vitro situation. When CCs fail to expand in culture dishes, it is often 
attributed to a lack of optimal conditions and factors that are required for activating genes that induce expansion, and often has no effect on the attainment of developmental competency (Murray, 2020).

For oocyte-specific genes associated with maturation and developmental competency, many functions in cell division and are associated with GVBD and the cell cycle such as stem-loop binding protein (SLBP), cyclins (CYCLINB1) and HSP9OB1. Many oocyte-derived genes are also correlated with successful oocyte-to-embryo transition and early embryonic development such as maternal genes (MATER) and zygote arrest 1 (ZAR1). Oocyte-secreted protein (OOSP1), GDF9 and BMP15 are also important oocyte-specific genes. OSFs are important paracrine signals that are crucial mediators of many key functions throughout oocyte development. The gene OOSP1 appears to influence oogenesis while GDF9 and BMP15 are key regulators of somatic cell functions such as expansion, apoptosis and proliferation (further discussed in Section 1.5.4). GDF9 has been shown to regulate amino acid and energy substrate uptake and transport to the oocyte. In mice, Gdf9 has also been correlated with CC expansion and induces the expression of Ptgs2 and Has2 in the presence of FSH (Elvin et al., 1999; Pangas et al., 2004). BMP15 has been shown to decrease apoptosis of $\mathrm{CC}$ within the $\mathrm{COC}$ by inducing the upregulation of chemokine ligand 2 (CCL2) and downregulating anti-apoptotic factors such as fibrillin-1 (FBN1) (Hussein et al., 2005; Zhai et al., 2013). Because these OSFs support many important cell functions, it is implied that OSFs heavily influence the expression of many of the other key genes associated with maturation and developmental competency.

The importance of GDF9 and BMP15 are also evidenced by reproductive defects in animals with mutations in these genes (discussed in section 1.4.5) and the ability of recombinant forms of these growth factors to mimic the actions of the oocyte on GC, CC and embryos (Reviewed in Section 1.5.6; Hussein et al., 2006). They have also demonstrated the improvement in developmental competency during IVM. Experiments conducted by Hussein et al. (2006) which co-cultured COC with denuded oocytes found increases in developmental competency, as the addition of exogenous OSFs secreted by the denuded oocytes in addition to the native OSFs endogenously secreted by the cultured COC had a synergistic effect in terms of improved blastocyst rate. 


\subsection{Bone Morphogenetic Protein 15 \& Growth Differentiation Factor 9}

\subsubsection{TGF- $\beta$ Superfamily of Proteins}

BMP15 and GDF9 are members of the transforming growth factor-beta (TGF- $\beta$ ) superfamily of proteins; a large group of structurally-conserved and widely-distributed polypeptides that control numerous physiological and developmental processes (Chang et al., 2002). The members of the superfamily have been classified into several subfamilies on the basis of additional conserved structural characteristics. These include the TGF- $\beta$ subfamily, the bone morphogenetic protein (BMP) subfamily, the growth differentiation factor (GDF) subfamily, the activin and inhibin subfamily of peptides and glial cell-derived neurotrophic factor (GDNF) subfamily. Additional members such as anti-Mullerian hormone $(\mathrm{AMH})$, nodal and lefty also belong to the TGF- $\beta$ superfamily (Sun \& Davies, 1995; Chang et al., 2002). Generally, most members of the superfamily are synthesised as a pre-pro-peptide precursor with an $\mathrm{N}$-terminal signal sequence that is followed by a large pro-region with regulatory folding functions, and a receptor-binding mature region at the C-terminus (Chang et al., 2002). These regions are enzymatically cleaved from each other into active proteins by furin-like proteases to form covalently-linked homodimers or heterodimers that bind their complementary signalling receptors. A characteristic cysteine motif in the core structure of the mature region consisting of six highly conserved cysteine residues, and a seventh involved in the formation of covalent bonds, stabilises the mature domain of these growth factors (McDonald \& Hendrickson, 1993; Sun \& Davies, 1995).

\subsubsection{Receptors of the TGF- $\beta$ Superfamily}

TFG- $\beta$ dimers form through a receptor-ligand complex following the binding of dimeric proteins to two type I and two type II receptors that have intrinsic Ser/Thr kinase activity (McPherron \& Lee, 1993). The type I receptors in mammals have several names and can be divided into three groups: (i) activin receptor-like kinase 4 (ALK4; activin receptor 1B, ActRIB), ALK5 (TGFBR1), and ALK7; (ii) ALK3 (BMPR-A) and ALK6 (BMPR1B); (iii) ALK1 and ALK2 (Chang et al., 2002; Knight \& Glister, 2003). The type II receptors contain kinase domains that are constitutively active. The formation of the receptor-ligand complex occurs upon the binding of a protein from the TGF- $\beta$ superfamily to its complementary 
type II receptor. The receptor phosphorylates and activates glycine and serine-rich domains of the type I receptor which constitutively recruits intracellular substrates to propagate a signalling cascade (Chang et al., 2002).

\subsubsection{Molecular Characteristics of GDF9 \& BMP15}

In contrast to other members of the TGF- $\beta$ superfamily, BMP15 and GDF9 are unusual in that they lack the seventh cysteine residue involved in the formation of the disulphide bond within the mature region. This seventh cysteine residue is instead substituted by a serine residue. As a result, these proteins do not readily dimerise and only do so in a noncovalent manner by maintaining a canonical dimer structure (Chang et al., 2002).

However, BMP15 and GDF9 still utilise their signals through a unique Type I receptor and a common Type II receptor. Activation of these receptors mediate intracellular Sma- and Mad-related (SMAD) proteins which regulate the transcription of various genes involved in GC proliferation and oocyte maturation (see Sections 1.4.4 and Table 1). The prodomain displacement, following the enzymatic cleavage of the pro and mature regions allows the BMP15 to form a complex with BMP receptor type II (BMPR2) and the type I receptor BMPR1B, and ultimately leads to the activation of the SMAD1/5/8 intracellular signalling pathway (Moore et al., 2003; Pulkki et al., 2012). Mature GDF9 can also bind to the same type II receptor as BMP15 (i.e. BMPR2) however, binds to the type I receptor TGF $\beta R 1$ which leads to the activation of the SMAD2/3 signalling pathway (Vitt et al., 2002; Kaivo-Oja et al., 2005).

Like other superfamily members, BMP15 and GDF9 are synthesised in a pre-pro-mature precursor form that is enzymatically cleaved into an active pro-mature form as they are secreted from the oocyte. The pro-mature proteins may be enzymatically processed further into mature forms. Whilst other superfamily members are shown to be secreted as mature homodimers and heterodimers, there is currently no evidence to suggest that these forms exist for GDF9 and BMP15 in vivo. However it is clear that non-covalent homodimers and heterodimers of the mature regions form at the level of their respective signalling receptors (Heath et al., 2017). 


\subsubsection{Role of GDF9 \& BMP15 in Somatic Cell Function}

In most species, GDF9 and BMP15 are believed to be largely restricted to the oocyte where they act synergistically to coordinate many functional roles during follicular growth. It has been established that during early follicular growth, they regulate granulosa cell (GC) proliferation and steroidogenesis, apoptosis, theca cell (TC) recruitment and oocyte maturation (Dong et al., 1996; Carabatsos et al., 1998; Hussein et al., 2005). Moreover, they play a different role during late follicular development where they are involved in the differentiation of GC to mural GCs and cumulus cells (CCs), the upregulation of EGFR expression in the $\mathrm{CC}$, as well as mucificaton and expansion of the CC and oocyte quality (Paulini \& Melo, 2011). Given the fact that the GCs of follicles in heterozygous BMP-mutant sheep acquire luteinising hormone receptor (LHR) precociously (McNatty et al., 2010; Crawford et al., 2011), they may indeed also regulate the timing of the acquirement of LHR on cattle GC.

The responsiveness of CCS to EGF is critical for the final stages of follicular maturation and has been reported to be reliant on a combination of oocyte-derived GDF9, BMP15 and cAMP (Sugimura et al., 2015). It has been shown that CCs of small antral follicles have a poor capacity for EGF/ EGFR signalling due to a deficiency in the level of production and/or secretion of GDF9 and BMP15. This unresponsiveness to EGF-like ligands results in poor developmental competency of oocytes within these immature follicles (Dunning et al., 2007; Dunning et al., 2012).

\subsubsection{Role of GDF9 \& BMP15 on Fertility}

It has been established that the relative expression of GDF9 and BMP15 mRNA varies significantly across different species but is highly conserved within species and that their relative ratios expressed by the oocyte represent a mechanism by which ovulation rate is regulated (Crawford \& McNatty, 2012). Species of a mono-ovulatory phenotype with lower fecundity tend to express a more balanced ratio of GDF9:BMP15 mRNA while species of a poly-ovulatory phenotype with higher fecundity express higher levels of GDF9 relative to BMP15 mRNA (Crawford \& McNatty, 2012). Studies conducted in various animal models have demonstrated the individual roles of these OSFs on the modulation of fertility. 
Experiments of mouse knock-out (KO) models have established that GDF9 is more essential in follicular development in poly-ovulatory rodent species than BMP15. Homozygous Gdf9-KO mice are sterile with follicular development arrested at the primary follicle stage resulting in high FSH and LH levels due to alterations in negative feedback loops, insufficient GC proliferation and loss of steroidogenesis regulation (Dong et al., 1996; Carabatsos et al., 1998). In contrast, the homozygous BMP15-KO mice demonstrated only sub-fertility, whereby although ovulation rate was slightly reduced, ovaries exhibited only subtle changes in histology and follicular development (Yan et al., 2001). Interestingly, experiments in transgenic mice overexpressing BMP15 mRNA showed accelerated follicular development in comparison to WT but did not demonstrate improvements in fecundity (McMahon et al., 2008). Double mutants of Gdf9 and Bmp15 $\left(\mathrm{Gdf9} \mathrm{S}^{+/-} \mathrm{Bmp15^{-/ } )}\right.$ were associated with a fragile cumulus oophorous following ovulation which significantly alters fertilisation, early embryonic development and its chances of implantation (Su et al., 2004). This demonstrates the functions of both the oocyte, CCs and overall development are impaired without a full complement of OSFs.

Studies on several breeds of sheep have demonstrated that natural mutations in GDF9 and BMP15 genes modulate fertility in these mono-ovulatory species. It has been shown in ewes that loss of either BMP15 or GDF9 gene can have different effects on ovulation rate (McNatty et al., 2001). Ewes of the Inverdale or Hanna genotype that carry a heterozygous mutation in the BMP15 gene have increased ovulation rate while double mutants are sterile. Moreover, Beclare ewes with heterozygous or homozygous mutations in GDF9 mRNA also exhibited an increased ovulation rate or sterility, respectively, similar to that observed in the genotypes with BMP15 mutations (Hanrahan et al., 2004). These different genotypes of sheep all demonstrate the importance of both GDF9 and BMP15 in fecundity. A natural mutation in the type I receptor for BMP15 (BMPR1B or ALK6) of Booroola ewes has also shown a single or double mutation in this gene has an additive effect on ovulation rate (Davis et al., 1991; Galloway et al., 2000). The double mutant Booroola ewe is particularly interesting as we would expect sterility with the complete loss in function of BMPR1B signalling. The increase in ovulation rate in double mutant Booroola demonstrates that BMP15 must act through alternative type I receptors that can form complexes with BMPR2 and non-SMAD signalling pathways to result in 
significantly improved fertility rates (Reader et al., 2011; Reader et al., 2016). However, while decreasing the signalling of GDF9 or BMP15 in a dose-dependent manner through either mutations in BMP15, GDF9 or their corresponding receptors increases ovulation rate, it is important to note that there is an evident threshold that must be met for GDF9 or BMP15 signalling. If follicles fall below this threshold (i.e. in the case of homozygous BMP15 or GDF9 mutants), follicular development ceases.

Studies by Crawford and McNatty (2012) found that mono-ovulatory animals (i.e. cows, red deer and sheep) had a lower GDF9:BMP15 mRNA ratio while poly-ovulatory animals (i.e. mice and rats) showed the contrary. An exception was the pig which exhibited appreciable expression levels of BMP15 in their oocyte, compared to GDF9, similar to that in mono-ovulatory animals (Paradis et al., 2009; Crawford \& McNatty, 2012). However, it was later established that high fecundity can be accounted for in pigs as their GC also express GDF9 mRNA (Christoforou, 2017). Therefore the ratio of GDF9:BMP15 protein, with which the granulosa cells are exposed to, may be similar to that of rodents given a lack of morphogenic gradient for GDF9 in the pig.

The importance of GDF9:BMP15 ratio appears to extend beyond GC proliferation rate. Supplementation of exogenous porcine GDF9 and BMP15 in IVM media for the maturation of ovine oocytes demonstrated that the addition of a higher GDF9:BMP15 ratio of 6:1 resulted in higher ovine developmental competency and improved blastocyst rate (Clark, 2018).

\subsubsection{Effects of Covalently-linked Dimers on Biological Functions}

Cumulin is a stable, covalently-linked heterodimer of BMP15 and GDF9 that was produced recombinantly in HEK293T cells (Mottershead et al., 2015). As stated previously, the formation GDF9-BMP15 heterodimers within biological fluids is yet to be proven although it is possible that they exist at low levels which are difficult to detect using current techniques such as Western blotting. Through plasmid expression in stable cell lines, multiple forms of mouse GDF9 (mGDF9) and human BMP15 (hBMP15) were produced to test the synergism of the different molecular forms (i.e. pro-mature and mature forms) of these two growth factors on GC proliferation rate and embryological outcome (Reader et al., 2011; Mottershead et al., 2015; Reader et al., 2016). Synergism was tested using 
various bioassays by comparing results following the addition of pro-mature mGDF9 and hBMP15 combined versus Ser-Cys mutants of pro-mature and mature GDF9 and BMP15 that were used to produce covalently bound heterodimers (pro-cumulin and cumulin, respectively) with the addition of hBMP15 homodimers as a dimer control. In silico proliferation bioassays were utilised to test the effects of these dimers on incorporation of $\left[{ }^{3} \mathrm{H}\right]$-thymidine into mural GC. The synergistic action of GDF9 and BMP15 was largest with pro-cumulin relative to when two pro-mature homodimer forms were added in combination. Interestingly, whilst cumulin was in fact more potent at stimulating GC proliferation rate and activation of genes involved in CC expansion, pro-cumulin was actually a more potent stimulator of oocyte developmental competency through increased CC expansion, and improvements in blastocyst and hatched blastocyst rate (Mottershead et al., 2015). 


\subsection{Research Aims}

IVM, though promising, falls short as a successful technology for assisted reproduction due its failure to mimic and maintain crucial signalling processes which occur within a physiological follicular environment. However, these issues can be perturbed with the addition of key components into culture media.

The purpose of this study was to examine three divergent ratios of GDF9:BMP15 (1:6, 1:1, 6:1) supplemented into IVM media on the maturation and developmental competence of ovine COCs.

COCs will be cultured in a modified multiphasic IVM system developed by the Pitman Lab at Victoria University of Wellington (Wellington, New Zealand), where biomarkers of maturation and developmental competence will be assessed at three different timepoints ( 1 hour, 7.5 hours and 24 hours) using a range of molecular and biochemical assays.

The assays included for the determination of maturation include: (i) observation of COC morphology through GVBD and CC expansion; (ii) GJ communication within the COC; (iii) intra-oocyte GSH levels; (iv) changes in glycolytic metabolites (glucose, pyruvate and lactate) in IVM media; (v) GC proliferation rates, and; (vi) expression levels of key oocyte and CC genes (Table 1).

We hypothesise that relative to the other ratios and a no-OSF control, the supplementation of IVM media with a 6:1 GDF9:BMP15 ratio would result in an overall improvement of oocyte maturation and developmental competency. 
Table 1: Table of biomarkers selected for gene expression analyses for this study.

\begin{tabular}{|c|c|c|}
\hline Gene & Function & References \\
\hline \multicolumn{3}{|l|}{ Oocyte Query Genes } \\
\hline $\begin{array}{l}\text { HIF1A } \\
\text { Histone-Induced Factor } 1 \alpha\end{array}$ & $\begin{array}{l}\text { Regulator of hypoxic response. Upregulated under } \\
\text { hypoxic conditions. Specific to oocyte. Blockage of HIF } \\
\text { suppresses ovulation and reduced antrum formation. }\end{array}$ & $\begin{array}{l}\text { (Douglas et al., 2005; } \\
\text { Kim et al., 2009; } \\
\text { Takahashi et al., } \\
\text { 2016) }\end{array}$ \\
\hline $\begin{array}{l}\text { SOD1 } \\
\text { Superoxide Dismutase } 1\end{array}$ & $\begin{array}{l}\text { An antioxidant enzyme which is closely associated with } \\
\text { BCL2. Increased expression in follicular fluid is associated } \\
\text { with follicular development and maturation. Reduced } \\
\text { expression of Sod1 in MII oocytes of mice reduces } \\
\text { developmental competence due to age-related decline in } \\
\text { oocyte quality. Knock-out Sod1 increases apoptosis and } \\
\text { superoxides in the cellular microenvironment. }\end{array}$ & $\begin{array}{l}\text { (Barbosa et al., 2010; } \\
\text { C. M. Combelles et } \\
\text { al., 2010; Tennant \& } \\
\text { Kligerman, 2011; } \\
\text { Saccon et al., 2013) }\end{array}$ \\
\hline $\begin{array}{l}\text { PRDX6 } \\
\text { Peroxiredoxin-6 }\end{array}$ & $\begin{array}{l}\text { A group of antioxidant enzymes that modulate oxidative } \\
\text { stress and DNA damage. Increased expression in both } \\
\text { bovine CC and oocytes. Upregulation over IVM is } \\
\text { associated with high developmental competence but only } \\
\text { in COC not denuded oocyte. CC contact is crucial. }\end{array}$ & (Leyens et al., 2004) \\
\hline $\begin{array}{l}\text { BMP15 } \\
\text { Bone Morphogenetic Protein } \\
15\end{array}$ & $\begin{array}{l}\text { An oocyte-secreted growth factor with a major role in } \\
\text { follicular development and maturation. Plays a key role in } \\
\text { preventing apoptosis by maintaining the gradient of anti- } \\
\text { apoptotic factors. }\end{array}$ & $\begin{array}{l}\text { (Galloway et al., } \\
\text { 2000; Hussein et al., } \\
\text { 2005; Hussein et al., } \\
\text { 2006) }\end{array}$ \\
\hline $\begin{array}{l}\text { GDF9 } \\
\text { Growth Differentiation Factor } \\
9\end{array}$ & $\begin{array}{l}\text { An oocyte-secreted growth factor with a major role in } \\
\text { follicular development and maturation. Plays a key role in } \\
\text { regulating CC, amino acid and energy substrate transport } \\
\text { to the oocyte. Known to upregulate expansion genes such } \\
\text { as HAS2, COX, PTGS2, GREM1. Supports the pre-ovulatory } \\
\text { LH surge. Improves blastocyst rate. }\end{array}$ & $\begin{array}{l}\text { (Hanrahan et al., } \\
\text { 2004; Hussein et al., } \\
\text { 2005; Hussein et al., } \\
\text { 2006) }\end{array}$ \\
\hline \multicolumn{3}{|l|}{ Cumulus Cell Query Genes } \\
\hline $\begin{array}{l}\text { EGFR } \\
\text { Epidermal Growth Factor } \\
\text { Receptor }\end{array}$ & $\begin{array}{l}\text { Increases in CC as the follicle progresses through } \\
\text { development. Key for EGF signalling which play a role in } \\
\text { triggering the resumption of meiosis. }\end{array}$ & $\begin{array}{l}\text { (Sugimura et al., } \\
\text { 2015) }\end{array}$ \\
\hline $\begin{array}{l}\text { BCL2 } \\
\text { B-cell Lymphoma } 2\end{array}$ & An anti-apoptotic factor. & (Dhali et al., 2017) \\
\hline $\begin{array}{l}\text { BAX } \\
\text { BCL2 Associated X }\end{array}$ & A pro-apoptotic factor. & (Hussein et al., 2005) \\
\hline $\begin{array}{l}\text { GSTA1 } \\
\text { Glutathione-S-Transferase } \alpha 1\end{array}$ & $\begin{array}{l}\text { An enzyme which conjugates GSH to thiols to detoxify the } \\
\text { cellular microenvironment. Decreased expression in COC } \\
\text { in IVM and in vivo. }\end{array}$ & $\begin{array}{l}\text { (Rabahi et al., 1999; } \\
\text { Salhab et al., 2011) }\end{array}$ \\
\hline $\begin{array}{l}\text { TNFAIP6 } \\
\text { Tumour Necrotic Factor } \\
\text { Alpha Induced Protein } 6\end{array}$ & $\begin{array}{l}\text { Involved in extracellular matrix formation, adhesion, cell } \\
\text { structure. Associated with CC expansion. }\end{array}$ & $\begin{array}{l}\text { (Hsieh et al., 2009; El- } \\
\text { Hayek et al., 2014) }\end{array}$ \\
\hline $\begin{array}{l}\text { FSHR } \\
\text { Follicle-Stimulating Hormone } \\
\text { Receptor }\end{array}$ & $\begin{array}{l}\text { A key receptor for FSH signalling which is important for } \\
\text { follicular development. Decreases in expression due to } \\
\text { luteinisation signalling from LH or hCG. }\end{array}$ & $\begin{array}{l}\text { (Ekart et al., 2013; } \\
\text { Dhali et al., 2017) }\end{array}$ \\
\hline $\begin{array}{l}\boldsymbol{P} \boldsymbol{R} \\
\text { Progesterone Receptor }\end{array}$ & $\begin{array}{l}\text { Associated with successful ovulation. Key in signalling of } \\
\text { progesterone which establishes and maintains corpus } \\
\text { lutea and pregnancy by mediating the oviduct } \\
\text { microenvironment. Rapidly and transiently induced in } \\
\text { response to LH or hCG. }\end{array}$ & $\begin{array}{l}\text { (Hasegawa et al., } \\
\text { 2005; Robker et al., } \\
\text { 2009) }\end{array}$ \\
\hline $\begin{array}{l}\text { CX43 } \\
\text { Connexin } 43\end{array}$ & $\begin{array}{l}\text { A key GJ protein particular between CC. Down-regulated } \\
\text { expression upon luteinisation of the follicle. }\end{array}$ & $\begin{array}{l}\text { (Wiesen \& Midgley, } \\
\text { 1993; Feuerstein et } \\
\text { al., 2007; Hasegawa } \\
\text { et al., 2007) }\end{array}$ \\
\hline \multicolumn{3}{|l|}{ Reference Genes } \\
\hline $\begin{array}{l}\text { RPL19 } \\
\text { Ribosomal Lipoprotein } 19\end{array}$ & Validated for use in human and rat oocytes. & $\begin{array}{l}\text { (Campen, 2013; Ekart } \\
\text { et al., 2013) }\end{array}$ \\
\hline $\begin{array}{l}\text { PPIA } \\
\text { Peptidylprolyl Isomerase A }\end{array}$ & Validated for use in bovine oocytes. & $\begin{array}{l}\text { (Macabelli et al., } \\
\text { 2014) }\end{array}$ \\
\hline
\end{tabular}




\section{CHAPTER 2: MATERIALS \& METHODS}

\subsection{Experimental Design}

The following experiments were designed to investigate the effects of supplementing into standard ovine IVM media, recombinant porcine GDF9 and BMP15 at the ratios of 1:1, $1: 6,6: 1$. Oocyte maturation was determined indirectly by evaluating accepted measures of oocyte maturation and developmental competency. Namely, (i) the degree of GJ communication within the COC, (ii) intra-oocyte GSH levels within the oocyte; (iii) morphological changes of the COC, (iv) consumption of glycolytic metabolites within IVM media, (v) biomarker genes within oocytes and CCs and, (vi) GC proliferation rate. These three treatments and an appropriate control (discussed below) were prepared in commercial Medium 199 (M199) with modifications that better mimic the changing microenvironment of the maturing follicle. All experiments were conducted using aseptic technique under sterile conditions.

Each experimental replicate consisted of eight COC/treatment group/timepoint. The time points selected for sample collection were at 1, 7.5 and $24 \mathrm{hr}$ of culture. Of the eight replicate COC collected per replicate experiment, six were analysed morphologically for the presence or absence of a GV (i.e. GVBD) and then for either GJ activity or intracellular GSH measurements. The remaining two COCs were collected for gene expression analyses and the IVM media was collected for determination of the changes in consumption and/or production of glycolytic intermediates (pyruvate). GCs were radiolabelled with tritiated thymidine and assessed for proliferation. 


\subsection{Biphasic Gold Standard Ovine IVM System}

A schematic diagram of the IVM system utilised in this study is depicted in Figure 4. It should be noted that this IVM system is the gold standard for maturing ovine oocytes in vitro, with modifications to better mimic the follicular microenvironment of a maturing follicle (Murray, 2017; Clark, 2018) The IVM M199 medium used as the base medium was a formulation provided by Gibco $®$ by Life Technologies, ThermoFisher, United States (Cat 11150-059) described in full in Section 2.2.1. Specifically, three additional supplements were added to the media. The addition of CNP (Sigma Aldrich, United States) inhibits spontaneous meiotic resumption; OVA2 (oFSH WAL; purified ovine FSH; McNatty et al. (2009)) assists with CC expansion and the attainment of developmental competency; and human chorionic gonadotrophin (Chorulon $®$ hCG; Provet NZ Pty Ltd, New Zealand) mimics the preovulatory LH surge and is the final signal for follicle maturation. For the first 7.5 hours of the incubation period, individual COCs were matured in M199 medium supplemented with OVA2 and CNP to final concentrations of $10 \mathrm{ng} / \mathrm{mL}$ and $1000 \mathrm{nM}$, respectively; and is described hereafter as Early IVM medium. The COCs were then transferred into M199 medium containing $10 \mathrm{ng} / \mathrm{mL}$ OVA2 and $10 \mu \mathrm{g} / \mathrm{mL}$ hCG for the remainder of the $24 \mathrm{hr}$ incubation period; and is described hereafter as Late IVM media. The transfer of the COC through the Early and Late IVM media implemented the goal of mimicking the changing microenvironment of the follicle as it grows from a medium antral follicle to a pre-ovulatory follicle.

All COC were individually cultured in $25 \mu \mathrm{L}$ droplets of IVM media. These droplets were produced by placing eight $12.5 \mu \mathrm{L}$ drops of control or treatment (containing GDF9 and BMP15) media onto the bottom of a $60 \mathrm{~mm}$ culture dish and covered with $8 \mathrm{~mL}$ mineral oil (Sigma Aldrich, United States). An additional $12.5 \mu \mathrm{L}$ of appropriate IVM media was then added to these droplets to produce a $25 \mu \mathrm{L}$ incubation droplet (Figure 4). These dishes were then placed in a $\mathrm{CO}_{2}$ incubator at $39^{\circ} \mathrm{C}, 5 \% \mathrm{CO}_{2}$ in air and $100 \%$ humidity for a minimum of 2 hours to equilibrate. 


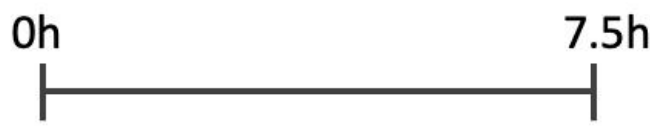

\section{EARLY M199 MEDIA}

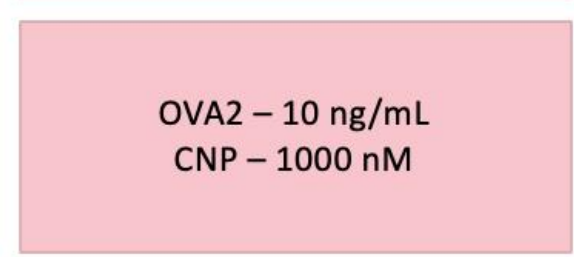

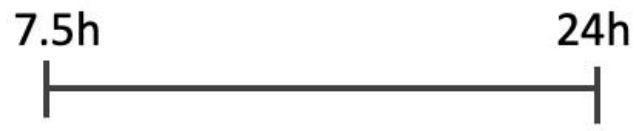

LATE M199 MEDIA

OVA2 - $10 \mathrm{ng} / \mathrm{mL}$ $\mathrm{hCG}-10 \mu \mathrm{g} / \mathrm{mL}$

Figure 4: Modifications to Gold Standard IVM incubation system. Depicts the changes in final concentrations of reagent supplementation in IVM medium.

\subsubsection{IVM Medium with GDF9:BMP15 Supplementation}

Regardless of treatment supplementation, all IVM media consisted of phenol-red M199 containing sodium bicarbonate for use in a high $\mathrm{CO}_{2}$ environment $\left(\mathrm{CO}_{2}\right.$ incubator) (Gibco $®$ by Life Technologies, ThermoFisher, United States) and supplemented $10 \%$ (v/v) charcoal-stripped foetal calf serum of NZ origin (Gibco $®$ by Life Technologies, ThermoFisher, United States). Media for bench work consisted of M199 powder (Gibco ${ }$ by Life Technologies, ThermoFisher, United States) supplemented with $0.1 \mathrm{mM}$ kanamycin (Sigma Aldrich, United States) and $3.3 \mathrm{mM}$ pyruvate. Additionally to control for changes in $\mathrm{pH}$ during dissections, media for bench work was supplemented with 25 mM HEPES (Sigma Aldrich, United States). Treatments consisted of differing ratios of recombinant porcine GDF9 and BMP15 (i.e. 1:6, 1:1 or 6:1, or an appropriate control) supplemented in IVM media (see Sections 2.2.2, 2.2.3., and Appendix 2). 


\subsubsection{Stock preparations}

A $33 \mathrm{mM}$ stock solution of pyruvate was prepared by solubilising pyruvate (Sigma Aldrich, United States) in sterile water. A $250 \mathrm{mM}$ stock solution of sodium bicarbonate was also prepared by solubilising sodium bicarbonate (Sigma Aldrich, United States) in sterile water. A $10 \mathrm{mM}$ cysteamine hydrochloride stock was prepared by solubilising cysteamine hydrochloride (Sigma Life Science, United States) in sterile water (Appendix 2). Foetal calf serum (FCS) of New Zealand origin was purchased from Gibco ${ }^{\circledR}$ by Life Technologies (ThermoFisher, United States). Non-polar compounds such as lipophilic (lipid-related) substances including certain growth factors, hormones including steroids and cytokines were removed from FCS by charcoal-stripping. This was achieved by supplementing FCS with $1 \%(\mathrm{w} / \mathrm{v})$ activated charcoal and 0.1\% (w/v) Dextran (Pharmacia, Sweden).

\subsubsection{Treatment Preparations}

The current study utilised secreted media from HEK-293 cells transfected with pEFIRES plasmid containing either porcine ( $p$ )GDF9, porcine ( $p$ )BMP15 or an empty vector control. The resultant secreted media containing recombinant pGDF9 and pBMP15 (of porcine origin) have been previously characterised within the Pitman laboratory (Alhussini, 2016; Swinerd, 2016). The GDF9:BMP15 ratios tested in this study were 1:6, 1:1, 6:1. These ratios are based on mRNA expression levels within oocytes of mammals of varying litter sizes. A high GDF9:BMP15 ratio (i.e. 6:1) represents levels in mammals with larger litter sizes such as pigs and rats (i.e. offspring/litter $=>6$ ) and is associated with high fecundity. A GDF9:BMP15 ratio of 1:1 represents expression levels in mammals with a low-medium litter size such as sheep; (i.e. offspring/litter =1-3). A low GDF9:BMP15 ratio (i.e. 1:6) represents levels of mammals with a low litter size such as red deer (i.e. offspring/litter is strictly 1) and is associated with low fecundity in mammals.

The concentrations of these recombinant proteins within the secreted media have been previously estimated by Western blotting using purified Escherichia coli (E. coli)-produced GDF9 and BMP15 as standards (Alhussini, 2016; Swinerd, 2016). The concentration of pGDF9 and pBMP15 within the secreted media batch used in this thesis were $0.94 \mathrm{ng} / \mu \mathrm{L}$ and $0.58 \mathrm{ng} / \mu \mathrm{L}$, respectively. These preparations are un-purified, hence it is important to note that these preparations may contain contaminants from the HEK-293 cells. To 
mitigate any possible effects from HEK-293 cell contaminants, the control COCs received the same volume of secreted media from HEK-293 cells, as the treated COCs, that were instead transfected with the empty vector (Table 2 ).

In regards to the molecular forms of the OSFs within the secreted media, Western blots revealed that both $p$ GDF9 and $p$ BMP15 secreted media batches contained a mixture of both pro-mature ( $50 \mathrm{kDa}$ ) and mature ( $20 \mathrm{kDa})$ forms (Appendix 1). This was important as pro-mature forms, compared with cleaved mature forms, of these proteins have been previously reported to be a more potent stimulator of oocyte developmental competency and improved blastocyst rates (Mottershead et al., 2015).

Table 2: Treatment preparation summary for IVM. The volumes $(\mu \mathrm{L})$ of HEK293 cell secretions containing porcine (p) GDF9, BMP15 or empty vector (EV) added to a total volume of $25 \mu \mathrm{L}$ for each treatment preparation. To mitigate any effects of HEK293 expressed media, an EV secreted media was used to ensure the same volume was added to each $25 \mu \mathrm{L}$ preparation.

\begin{tabular}{lcccc}
\hline & & \multicolumn{3}{c}{ GDF9:BMP15 Ratio } \\
\hline pGDF9 & Control & $\mathbf{1 : 6}$ & $\mathbf{1 : 1}$ & $\mathbf{6 : 1}$ \\
$(0.94 \mathrm{ng} / \mu \mathrm{L})$ & & 0.44 & 0.44 & 2.66 \\
pBMP15 & & & & \\
$(0.58 \mathrm{ng} / \mu \mathrm{L})$ & & 4.31 & 0.72 & 0.72 \\
EV & & 0 & 3.59 & 1.37 \\
Media & 4.75 & 20.25 & 20.25 & 20.25 \\
Total Vol $(\mu \mathrm{L})$ & 20.25 & 25 & 25 & 25 \\
\hline
\end{tabular}

\subsubsection{Ovary Dissection and Primary Cell Collection}

For IVM studies, ovaries from ewes of unknown ages and background were supplied from a local abattoir in Wellington, New Zealand. These were harvested for cells within 2 hours of being removed from the ewe. To reduce bacterial contamination, all ovaries were briefly rinsed in $70 \%(\mathrm{v} / \mathrm{v})$ ethanol, followed by two washes in $0.9 \%(\mathrm{w} / \mathrm{v})$ saline solution, in which they were stored at $37^{\circ} \mathrm{C}$ until primary cell collection. All washing solutions and dissection media had been prewarmed to $37^{\circ} \mathrm{C}$ for at least 1 hour prior to use. 
There are two common methods that can be utilised for COC collection; namely aspiration and plate dissection. While the aspiration method is ideal in terms of collecting more COCs in a shorter time frame, a previous study in the Pitman laboratory revealed that a less aggressive method results in better quality COCs. This plate dissection method demonstrated that following extraction, the COCs re-established the GJ connections faster and exhibited markedly higher levels of GJ communication levels relative to COC that had been extracted by aspiration (Ward, 2018). As this study is reliant upon the oocyte receiving optimal support from the $\mathrm{CC}$, the least disruptive $\mathrm{COC}$ extraction method (i.e. dissection extraction method) was used for the following experiments.

For the dissection extraction method, extraneous tissues on the ovaries were trimmed and discarded. Individual ovaries were then placed in a $90 \mathrm{~mm}$ culture dish and dissected down the midline. Half an ovary was transferred to a fresh $65 \mathrm{~mm}$ culture dish containing pre-warmed dissection media comprising of M199 (Gibco $^{\mathrm{TM}}$, ThermoFisher Scientific, United States of America) supplemented with $0.1 \mathrm{mM}$ kanamycin, $25 \mathrm{mM}$ HEPES, 500 IU/mL Heparin and $10 \%(\mathrm{w} / \mathrm{v})$ fatty acid free BSA (Appendix 2). Visible follicles between the sizes of 1-5 mm in diameter were punctured using a 20-gauge needle attached to a 3 $\mathrm{mL}$ syringe. The follicular contents were flushed gently with dissection media to encourage the release of the COC. Following dissection, COC were transferred to a $35 \mathrm{~mm}$ petri dish consisting of pre-warmed dissection media with the additional supplementation of $1000 \mathrm{nM}$ CNP and stored for no more than 20 minutes. The COCs with at least five intact layers of CC with a starting grade of 1 or 2 (further discussed in Section 2.5.2) were briefly washed in a $35 \mathrm{~mm}$ petri dish containing pre-warmed IVM media supplemented with $10 \% \mathrm{FCS}$, then transferred into single cell culture droplets of control or treatment Early IVM media.

All media was pre-warmed in a $37^{\circ} \mathrm{C}$ water bath and all experiments requiring bench work were conducted on a $38.5^{\circ} \mathrm{C}$ hot plate for consistency and temperature control. 


\subsection{Gap Junction Communication Assays}

\section{Experimental Overview}

A
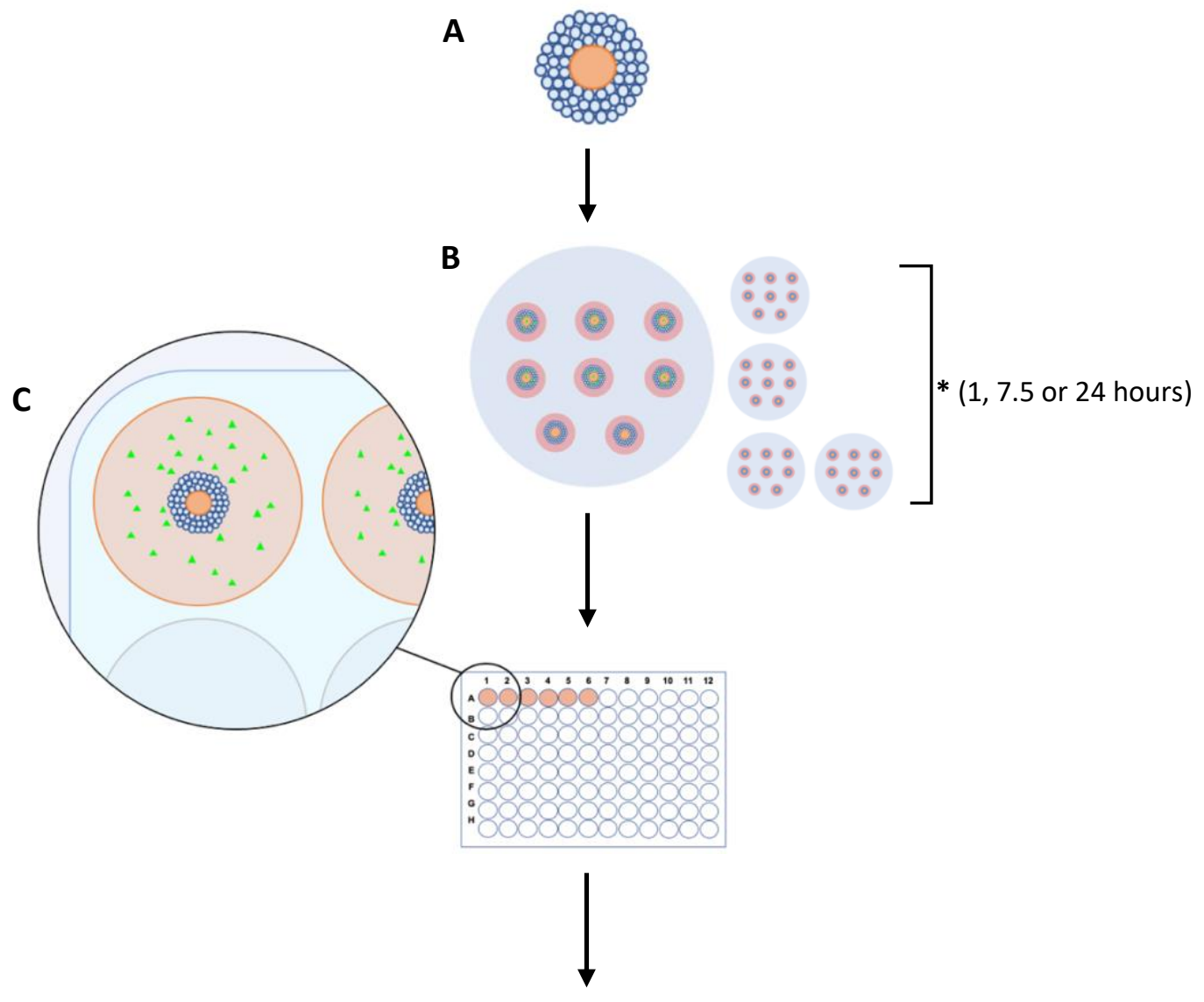

D

() 0

( 0 (

(6) 0

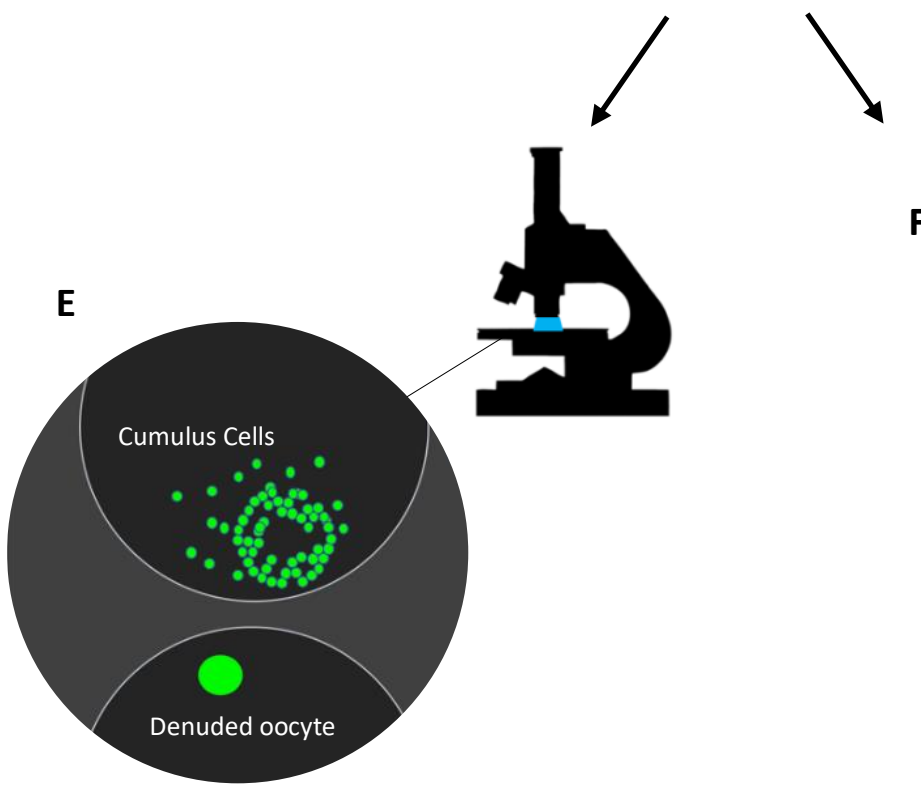

F 
Figure 5: Experimental overview for gap junction communication assay. [A]. COC are collected via dissection methods from ovine ovaries [B]. 8 COC of CC Grade 1 or 2 are individually cultured in $25 \mu \mathrm{L}$ incubation droplets according to IVM system and incubated at $39^{\circ} \mathrm{C}$ for the required time. Each OSF treatment requires 4 plates as depicted by $\left({ }^{*}\right)$ corresponding to incubation endpoints (1, 7.5 and 24 hours from top to bottom) and media changes for the 24 hour incubations. [C]. One hour prior to incubation endpoint, 6 COC are washed in calcein-AM for 10 minutes. [D]. COCs placed into droplets and incubated for final hour until endpoint reached. [E]. COCs are graded, denuded and oocytes observed for GV presence and measured for fluorescence to determine GJ activity. [F]. Two COC not washed in calcein-AM are denuded. Individual oocyte and corresponding CC are collected for RNA extraction, cDNA synthesis and gene expression analyses via QPCR. Media collected for assessment of metabolites. Not to scale.

Intracellular gap junction communication between the CC and oocyte during IVM was quantified by measuring the transfer of calcein-acetoxymethyl (calcein-AM) from the surrounding CC into the oocyte as previously reported (Campen, 2013). Calcein-AM is an AM ester derivative of the carboxylic acid and fluorescent dye calcein. This modification produces a molecule that is non-fluorescent, neutrally-charged and lipophilic. These molecular properties allow the dye to permeate the membranes of the $\mathrm{CC}$ in the outermost layer of the COC. Upon entering the cytoplasm of the CC, intra-cellular esterases hydrolyse the ester groups on the dye in a non-specific fashion, resulting in a molecule that is fluorescent and lipophilic. This product passively diffuses through the CC into the oocyte via GJ as depicted in Figure 6. The total transfer of calcein is quantified by the intensity of fluorescence in the oocyte (Figure 7; Figure 8). The intra-oocyte fluorescent levels are an indirect measure of the degree of gap junction communication between the CC and oocyte. The fluorescence levels were measured from the centre of each denuded oocyte (i.e. CC were removed) at a wavelength of $\sim 550 \mathrm{~nm}$ for $50 \mathrm{~ms}$ on a Leica inverted microscope (CTR4000) using a CRAIC fluorescent detection system and software (model QDI302, Leica Microsystems; Welzer, Germany) as previously described by Ward (2018). 


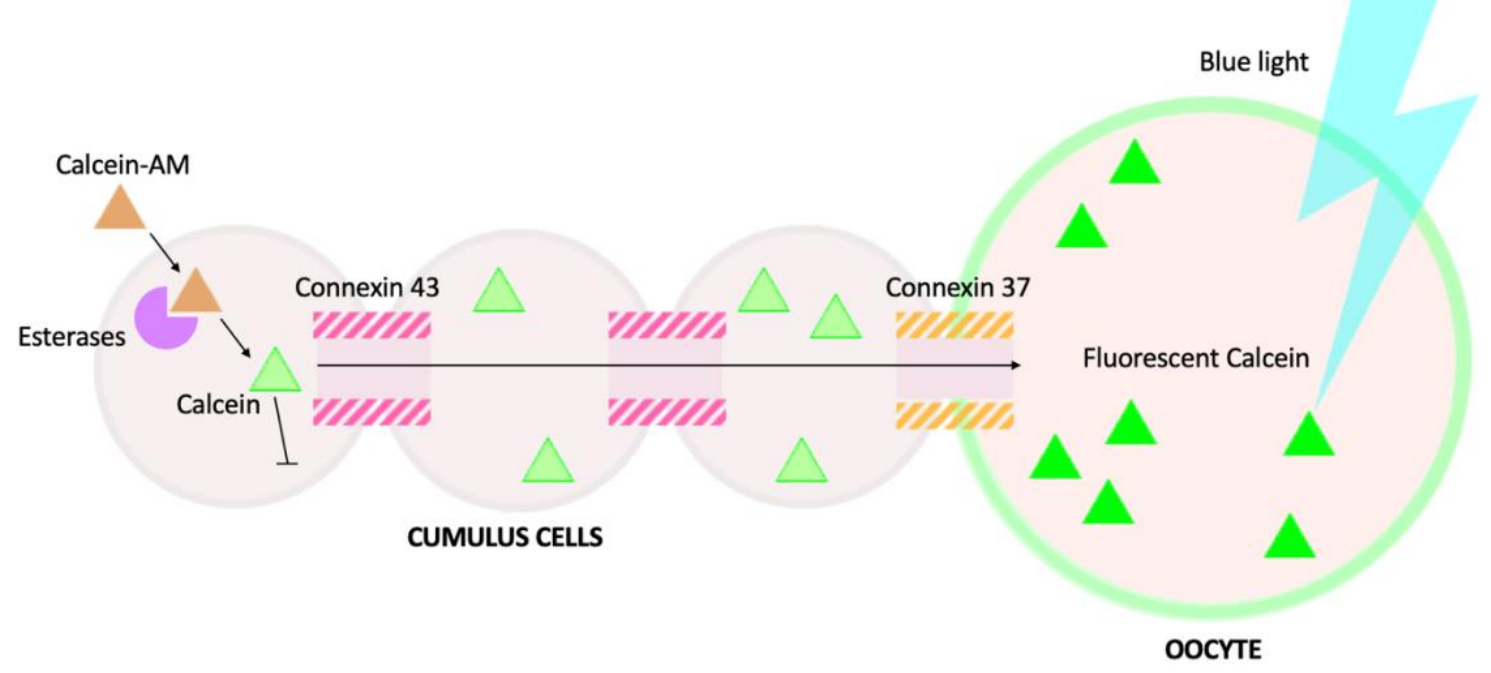

Figure 6: Schematic diagram of gap junction communication that indirectly measures communication between CC and oocyte. Calcein-AM permeates the membrane of outermost CC. Upon entrance into the CC, the AM group is cleaved by non-specific esterases, leaving an insoluble, fluorescent calcein molecule that must move through GJs to the oocyte through passive diffusion. Direct blue light emission excites the calcein molecule at a wavelength of $\sim 550 \mathrm{~nm}$ causing the cells containing calcein to fluoresce green. Adapted with permission from Ward (2018). Not to scale. 


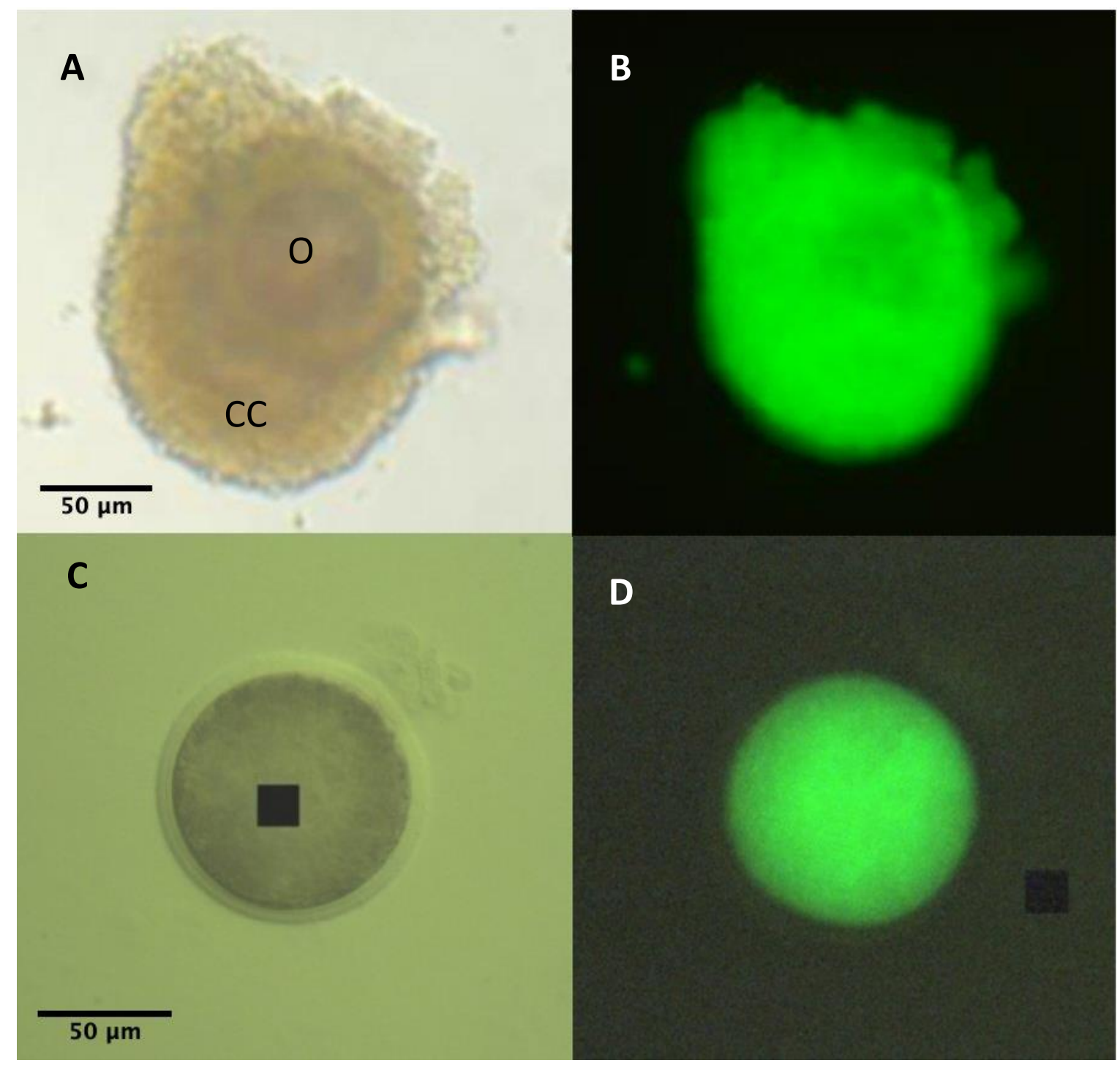

Figure 7: Microscopic images of bovine cumulus-oocyte complexes (COC) and denuded oocyte before and after exposure to calcein-AM. Bovine $\mathrm{COC}[\mathrm{A}, \mathrm{B}]$ before $[\mathrm{A}]$ and after [B] exposure to calcein-AM. Bovine denuded oocyte [C, D] before [C] and after [D] exposure to calcein-AM. Cumulus cell (CC) and oocyte (O) depicted in [A] (unpublished data, results not shown).

\subsubsection{Incubation Time Optimisation}

To determine the three timepoints during the incubation period where GJ activity, GSH levels and metabolites would be measured, a time series was conducted over 24 hours in a control IVM system (i.e. containing no OSF supplementation). This was optimised using a GJ assay as its use has been well established in the Pitman lab, whilst the GSH assay has only recently been optimised for the COC (Ward, unpublished results). Whilst it was known that two of these three timepoints would be 1 hour and 24 hours, it was difficult to determine an intermediate point at which GJ activity would eventually begin to reach 
a saturation point, particularly between 12 and 24 hours. We decided to observe activity at 7.5 hours as this is the point of transfer from the Early to Late media, and 16 hours as this is where we predicted any treatment effects may be evident. As an extra measure to decipher more information over a 24 hour period, a 4 and 20 hour timepoint was also included.

An hour prior to the final incubation time (i.e. at 3, 6.5, 15, 20 and 23 hours for the 4, 7.5, 16,20 and 24 hour incubation times respectively), or immediately after extraction in the case of the 1 hour timepoint, the eight COC were transferred into individual wells of a 96well plate and washed twice in $20 \mu \mathrm{L}$ of pre-warmed IVM media containing M199 supplemented with 10\% FCS for the purpose of removing any residual mineral oil. A 0.4 $\mu \mathrm{L}$ aliquot of $1 \mathrm{mM}$ calcein-acetoxymethyl (AM) was added to $20 \mu \mathrm{L}$ of the appropriate incubation media for a final concentration of $20 \mu \mathrm{M}$. The individual COCs were then transferred into a separate well containing $20 \mu \mathrm{L}$ of $20 \mu \mathrm{M}$ calcein-AM for 10 minutes. The COC were then transferred back to their original incubation droplet and incubated for a further hour to enable the transfer of dye through the CC layers and into the oocyte via GJs. Thus, the final incubation times were 1, 4, 7.5, 16, 20 and 24 hours. Following completion of the incubation period, the COCs were transferred through two individual wash wells of a 96-well plate containing $20 \mu \mathrm{L}$ of pre-warmed PBS (Gibco ${ }^{\mathrm{TM}}$, ThermoFisher Scientific, United States of America) to remove residual mineral oil. The COCs were then transferred to another well containing $20 \mu \mathrm{L}$ of PBS and repeated pipetting of COCs stripped the CC from the oocytes. The denuded oocyte was then placed into a separate well of $20 \mu \mathrm{L}$ PBS for fluorescence measurements.

Once the final three timepoints were established (1, 7.5 and 24 hours), they were used consistently as endpoints in all other assays, to collect media samples, and primary cells for QPCR.

\subsubsection{Experimental Gap Junction}

An hour prior to the final incubation time (i.e. immediately after extraction, at 6.5 and 23 hours for the 1, 7.5 and 24 hour incubation times, respectively), six of the eight COCs in each treatment group were transferred into individual wells of a 96-well plate and washed twice in $20 \mu \mathrm{L}$ of the appropriate pre-warmed IVM media for the purpose of removing any 
residual mineral oil. A $0.4 \mu \mathrm{L}$ aliquot of $1 \mathrm{mM}$ calcein-acetoxymethyl (AM) was added to $20 \mu \mathrm{L}$ of the appropriate IVM media for a final concentration of $20 \mu \mathrm{M}$. The individual COCs were then transferred into a separate well containing $20 \mu \mathrm{L}$ of $20 \mu \mathrm{M}$ calcein-AM for 10 minutes. The $\mathrm{COC}$ were then transferred back to their original incubation droplet and incubated for a further hour to enable the transfer of dye via gap junctions. Thus, the final incubation times were $1,7.5$ and 24 hours. Following the completion of the incubation period, the COCs were transferred through two individual wash wells of a 96well plate containing $20 \mu \mathrm{L}$ of pre-warmed PBS to remove residual mineral oil. The COCs were then transferred to another well containing $20 \mu \mathrm{L}$ of PBS and stripped of CC via repeated pipetting. The denuded oocyte was then placed into a separate well of $20 \mu \mathrm{L}$ PBS for fluorescence measurements and determination of meiotic resumption on the basis of GV presence (further discussed in Section 2.5.1). A total of four biological replicate experiments were conducted.

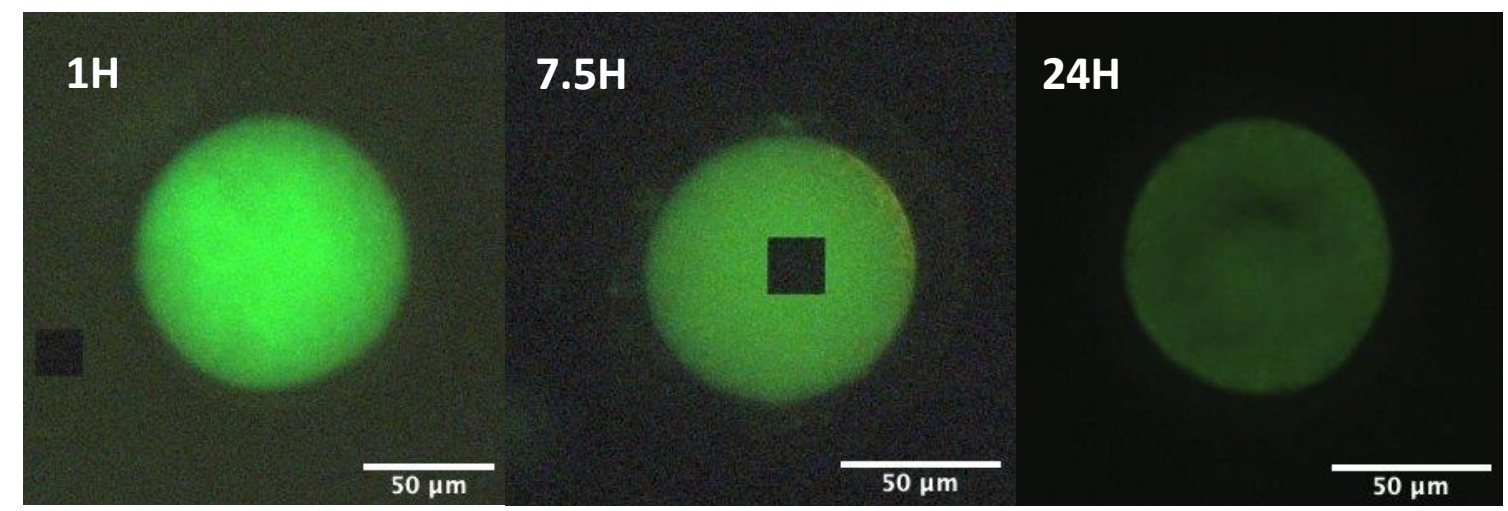

Figure 8: Fluorescence levels in ovine denuded oocytes as an indirect level of bidirectional intra-oocyte communication via GJs. Time 1, 7.5, 24 hours as depicted from left to right. The decrease in fluorescence indicates the closure of GJs that is associated with maturation of the oocyte. 


\subsection{Glutathione Conjugation Assay for Oxidative Metabolism}

\section{Experimental Overview}

A
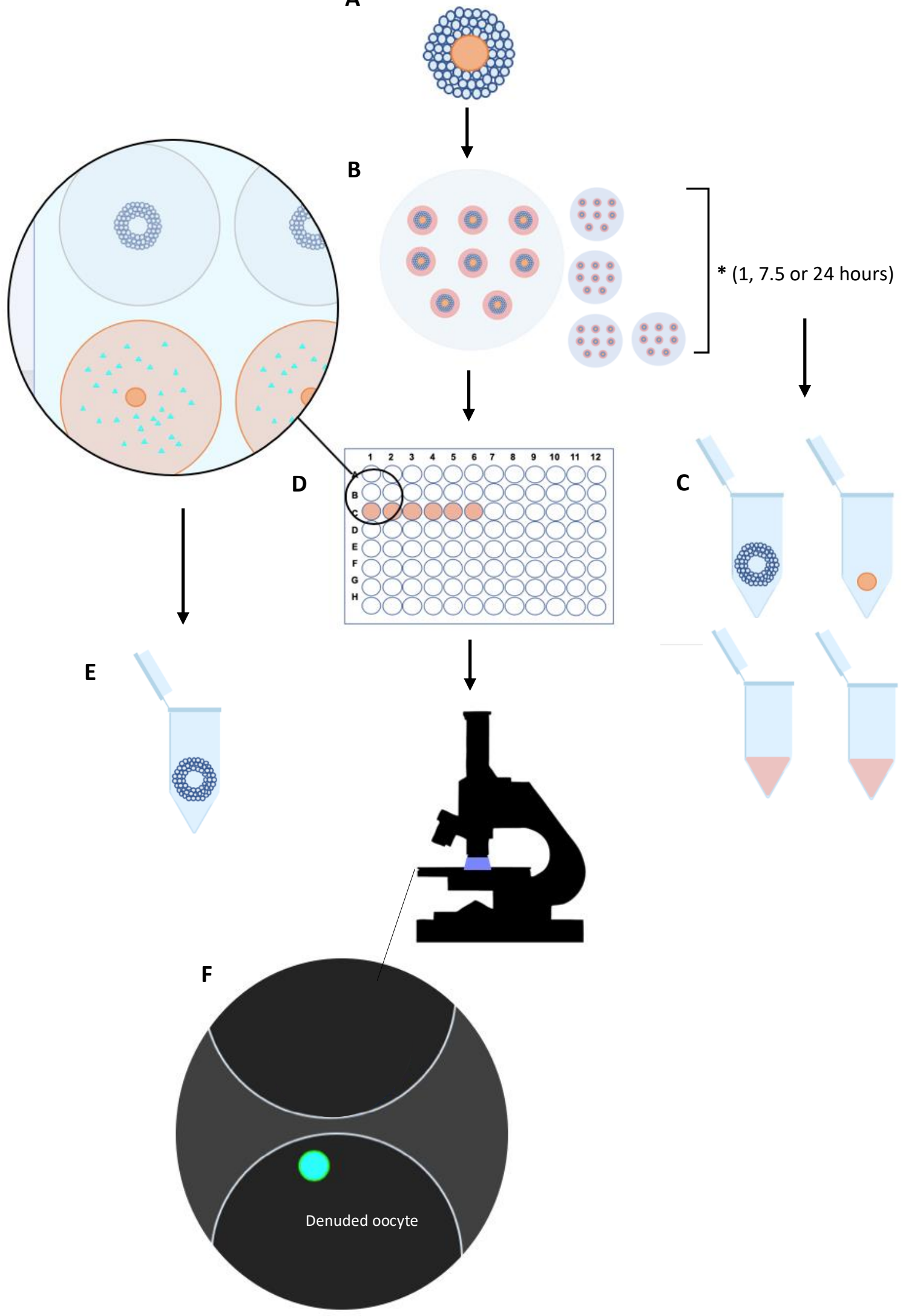
Figure 9: Experimental overview for glutathione assay. [A]. COC were collected via dissection methods from ovine ovaries [B]. 8 COC of CC Grade 1 or 2 were individually cultured in $25 \mu \mathrm{L}$ incubation droplets according to IVM system and incubated at $39^{\circ} \mathrm{C}$ for the required time. Each OSF treatment required four plates as depicted by $\left({ }^{*}\right)$ corresponding to incubation endpoints ( $1,7.5$ and 24 hours from top to bottom) and media changes for the 24 hour incubations. [C]. At the end of the incubation endpoint, two COC are denuded. Individual oocyte and corresponding CC are collected for RNA extraction, cDNA synthesis and gene expression analyses via QPCR. Media collected for assessment of metabolites. [D]. Remaining six COC are graded, denuded and placed into individual wells of a 96-well plate containing $\mathrm{MCB}$ and incubated for one hour. [E]. Stripped CC are also collected for RNA extraction, cDNA synthesis and gene expression analyses. [F]. Denuded oocytes are observed for GV presence and measured for fluorescence to determine GSH levels. Not to scale.

Thiols are important molecules which biologically function as antioxidants. They are capable of preventing damage to crucial cellular organelles and compartments by facilitating the metabolism of lipophilic xenobiotics such as ROS (e.g.. free radicals, peroxides and heavy metals), therefore maintaining the overall homeostasis of cellular redox state. Glutathione is the most abundant thiol in the animal cell and exists in both a reduced (GSH) and oxidised (GSSG) state. The ratio of GSH:GSSG can be used as an indirect measure of overall cellular oxidative stress (Aquilano et al., 2014). The conjugation of thiols to xenophilic molecules is catalysed by the glutathione-S-transferase (GST) family of enzymes.

Intracellular levels of GSH in oocytes was quantified by measuring the level of conjugation of monochlorobimane (MCB) to glutathione present within the cytoplasm as previously reported in neuronal studies conducted by Keelan et al. (2001). This methodology was further optimised in the Pitman lab for the mammalian COC (Ward, unpublished results). MCB is a lipophilic, non-fluorescent, cell permeable probe which is readily conjugated by the GST family of enzymes to low molecular weight thiols with high specificity to GSH. The GSH-MCB conjugate (GSB) is fluorescent (Figure 10).

Total intra-oocyte GSH is quantified by the intensity of fluorescence in the oocyte (Figure 11). The fluorescence levels were measured from the centre of each denuded oocyte at a wavelength of $\sim 490 \mathrm{~nm}$ for $50 \mathrm{~ms}$ on a Leica inverted microscope (CTR4000) using a CRAIC fluorescent detection system and software (model QDI302, Leica Microsystems; Welzer, Germany) as described by Ward (unpublished results). 


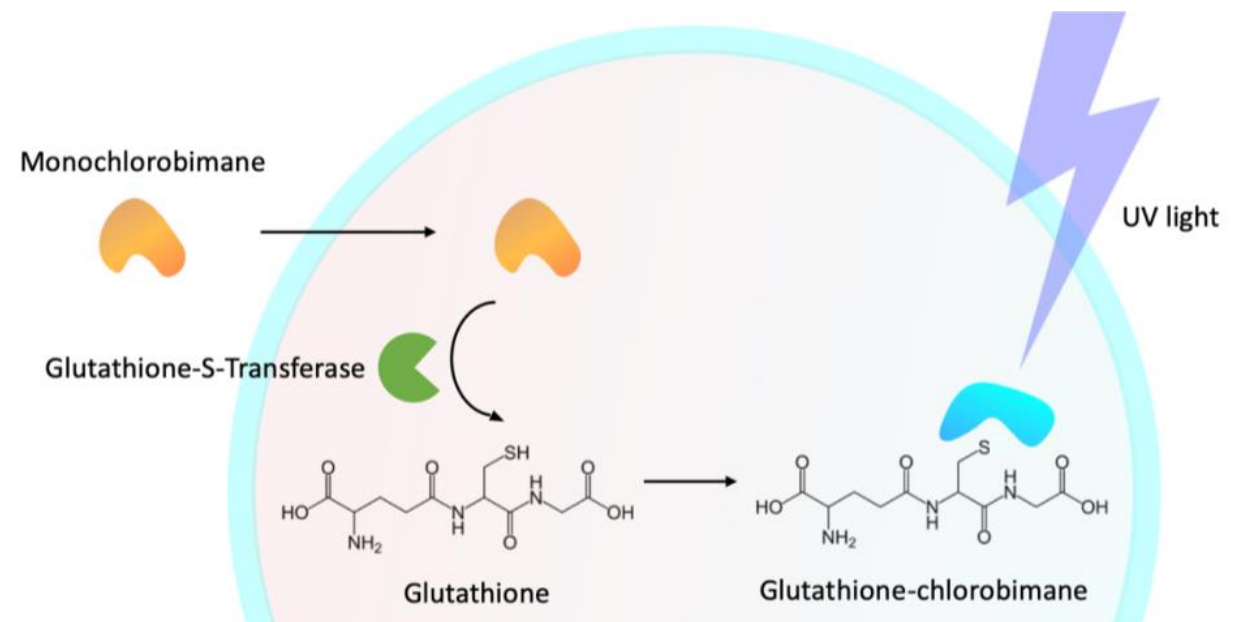

DENUDED OOCYTE

Figure 10: Schematic diagram depicting the conjugation of monochlorobimane (MCB) to reduced glutathione (GSH). MCB permeates the oocyte membrane and is conjugated to GSH by the glutathione-S-transferase (GST) family of enzymes. Direct UV emission excites the conjugated glutathione-chlorobimane (GCB) molecule at a wavelength of $\sim 490 \mathrm{~nm}$ causing the oocytes containing GCB to fluoresce blue. Not to scale.

As each final incubation time was completed (i.e. 1, 7.5, 24 hours), six of the eight COC in each treatment group were transferred to a 96-well plate containing $20 \mu \mathrm{L}$ of pre-warmed PBS. These COC were stripped of their CC via repeated pipetting. The stripped CC, in addition to the remaining two COC, were transferred into $0.6 \mathrm{~mL}$ Eppendorf tubes for further processing as described in Section 2.7.1. The denuded oocyte was transferred to another well containing $20 \mu \mathrm{L}$ PBS as an additional wash step. From this point onwards, the following steps were performed under minimal light exposure (red-light). An aliquot of $20 \mu \mathrm{L}$ of IVM media containing MCB at a final concentration of $12.5 \mu \mathrm{M}$ was added to a new well containing the denuded oocyte. The plate was further incubated for an hour at $39^{\circ} \mathrm{C}, 5 \%$ in air $\mathrm{CO}_{2}$ and $100 \%$ humidity. Following incubation, the denuded oocyte was transferred to a new well containing $20 \mu \mathrm{L}$ of pre-warmed PBS for fluorescent 
measurements and morphological assessment, as further discussed in Section 2.5.1. A total of three biological replicate experiments were conducted.

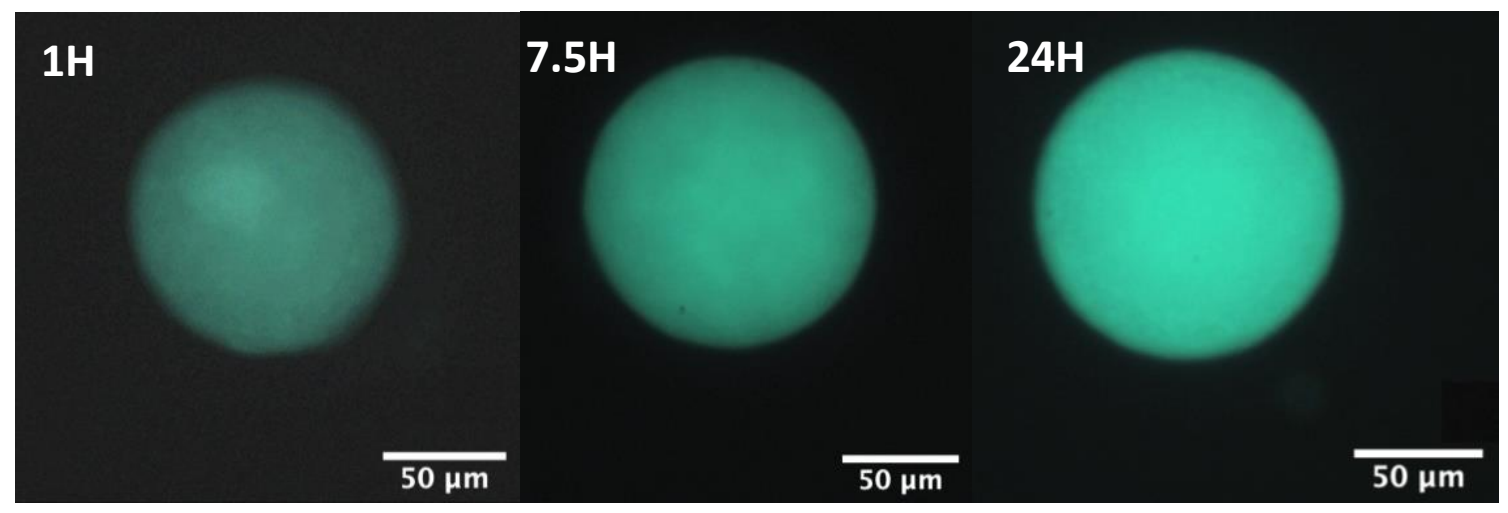

Figure 11: Fluorescence levels in ovine denuded oocyte as an indirect measure of reduced glutathione (GSH) in the ooplasm. Incubation times of 1, 7.5, 24 hours are depicted from left to right. The increase in fluorescence indicates higher rates of conjugation of the probe to GSH due to increased production levels in the oocyte. 


\subsection{Morphological Assessment for Maturation}

COC quality and level of maturation can be physically assessed based on its morphology. A morphological assessment was determined by the presence or absence of a GV at each incubation endpoint during the IVM experiments. Cumulus cell expansion was also used as a determinant.

\subsubsection{Germinal Vesicle Breakdown \& Meiotic Resumption}

The presence or absence of the GV is a direct determinant of the meiotic state of the oocyte, with its presence indicative of meiotic arrest while its absence was assumed to be due to GVBD and that meiosis has resumed (Figure 12).

The GV or GVBD status can be observed under a high power objective using a Leica Microscope CRAIC system. This data was typically recorded prior to the collection of fluorescent readings for GJ and GSH assays. However, this does have its limitations as the GV is not always obvious, particularly if there were vacuoles present in the ooplasm. Interestingly, when present the GV could be stained by the respective fluorescent probes (i.e. calcein and $\mathrm{MCB}$ ) and were clearer in contrast to the ooplasm, which could be used to overcome this obstacle (Figure 13).

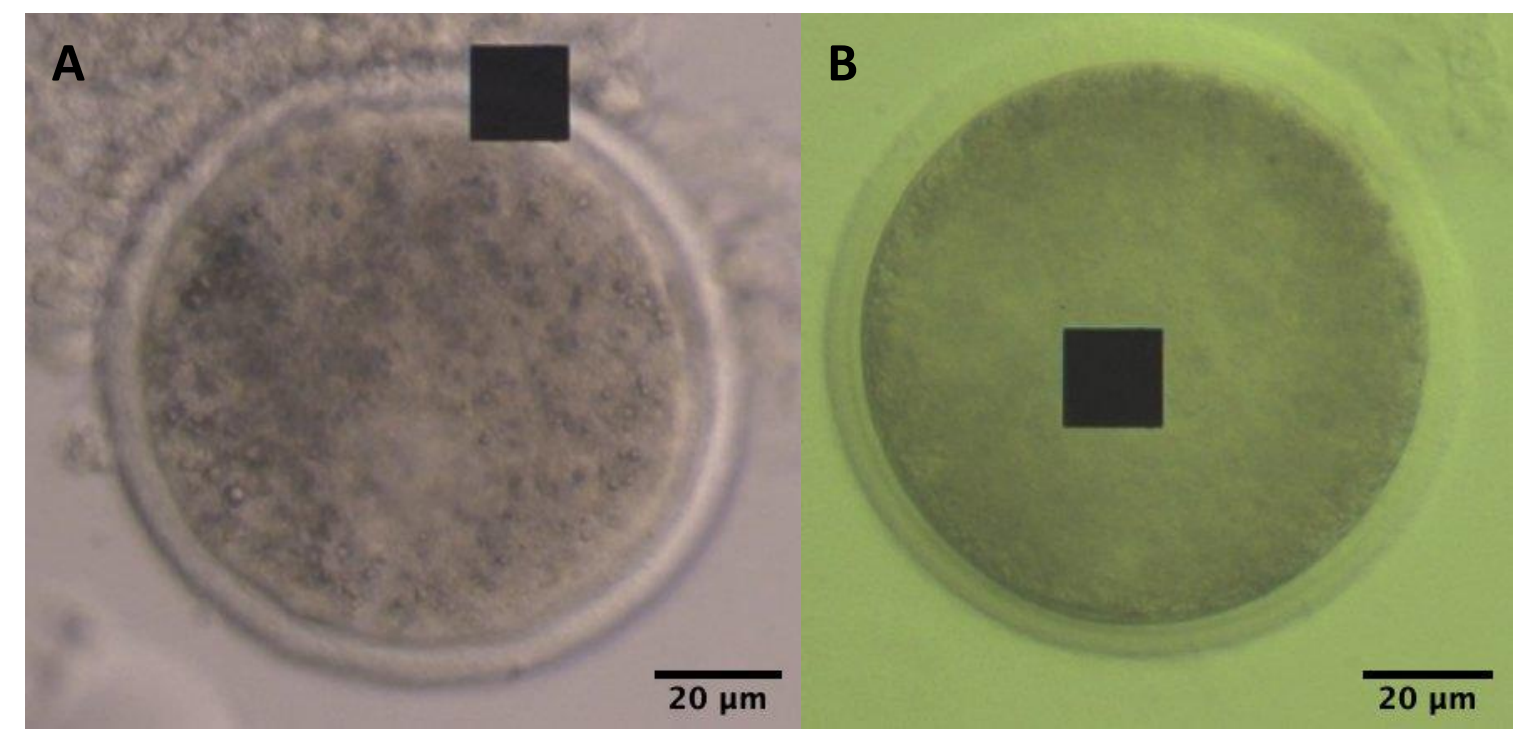

Figure 12: Germinal vesicle (GV) and germinal vesicle breakdown (GVBD) in ovine denuded oocyte. GV in denuded oocyte under high power objective. [B] GV in denuded oocyte stained by calcein-AM during a GJ communication assay. [C] GV in denuded oocyte stained by monochlorobimane (MCB) during a GSH conjugation assay. 


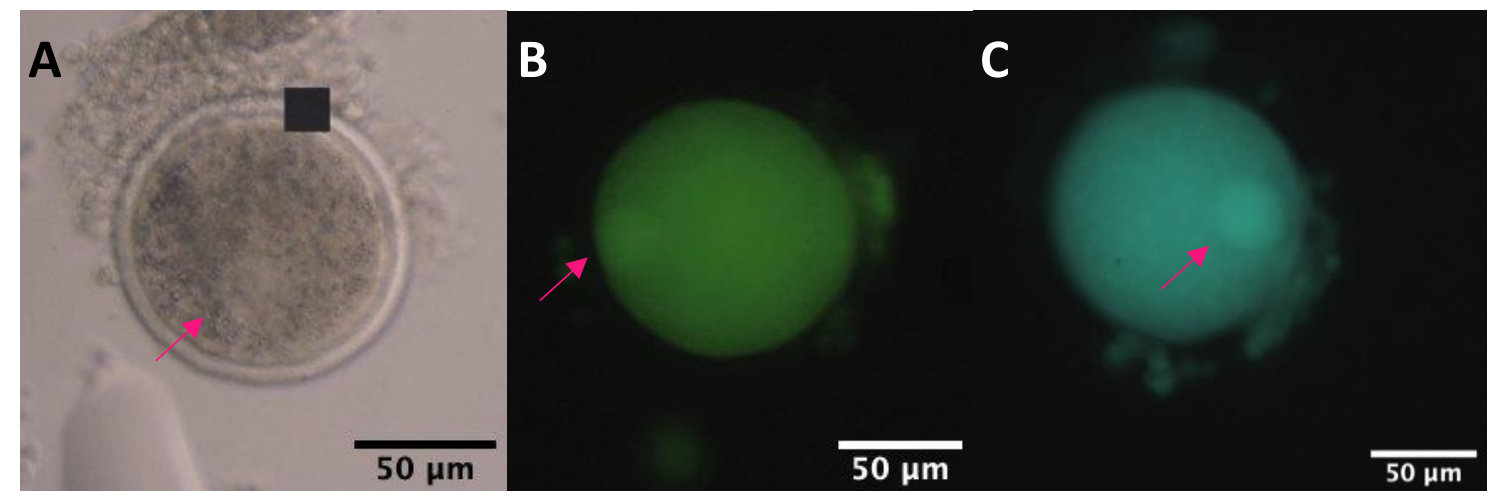

Figure 13: Germinal Vesicle (GV) in ovine denuded oocyte with and without probe staining. [A] GV in denuded oocyte under high power objective. [B] GV in denuded oocyte stained by calcein-AM during a GJ communication assay. [C] GV in denuded oocyte stained by monochlorobimane (MCB) during a GSH conjugation assay. GV depicted by arrow.

\subsubsection{Cumulus Cell Expansion}

The CCs surrounding the $\mathrm{COC}$ were graded at the time of collection and at the end of each incubation endpoint before denudation based on a system used by Clark (2018). The CC were graded during collection as only COC with cells graded 1 or 2 (Table 3 ) were selected for culture. However, it is important to note that the status of CC changes over the course of IVM (Figure 14). This may be due to multiple factors including expansion, oocyte health, influences of the OSFs on GJ communication and mechanical transfer from early to late IVM medium. While this process is gentle, the mechanical action of moving the COC can disrupt communication within the COC due to the closure of GJs.

Table 3: Cumulus cell grading criteria. CC grading scale determining level of CC expansion based on system utilised by Clark (2018).

\begin{tabular}{cl}
\hline Grade & Appearance \\
\hline $\mathbf{1}$ & Many layers of compact CC \\
\hline $\mathbf{2}$ & CC layers are fewer and partially expanded \\
\hline $\mathbf{3}$ & Fully expanded CC and mature oocyte \\
\hline $\mathbf{4}$ & CR only or denuded oocyte (no CCs) but ooplasm appears normal \\
\hline $\mathbf{5}$ & Degenerate; ooplasm is degenerate, absent or lysed. Expanded-atretic oocytes. \\
\hline
\end{tabular}


COMPACT

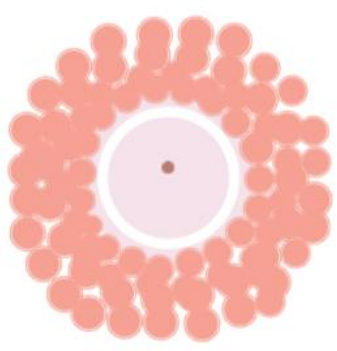

EXPANDED

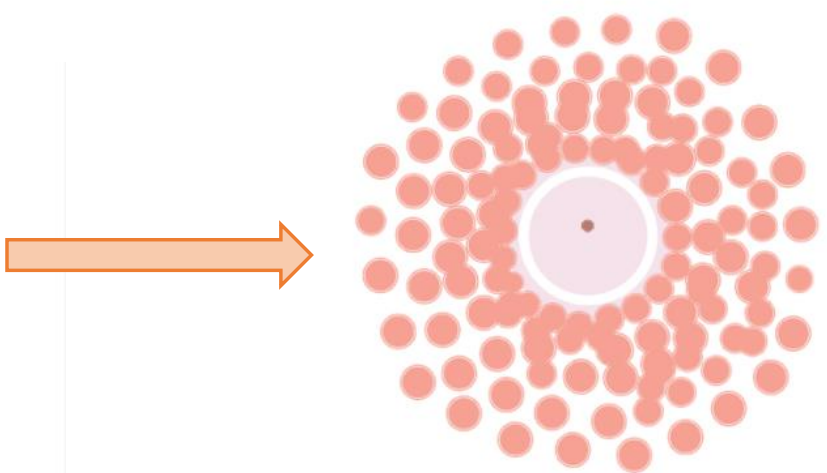

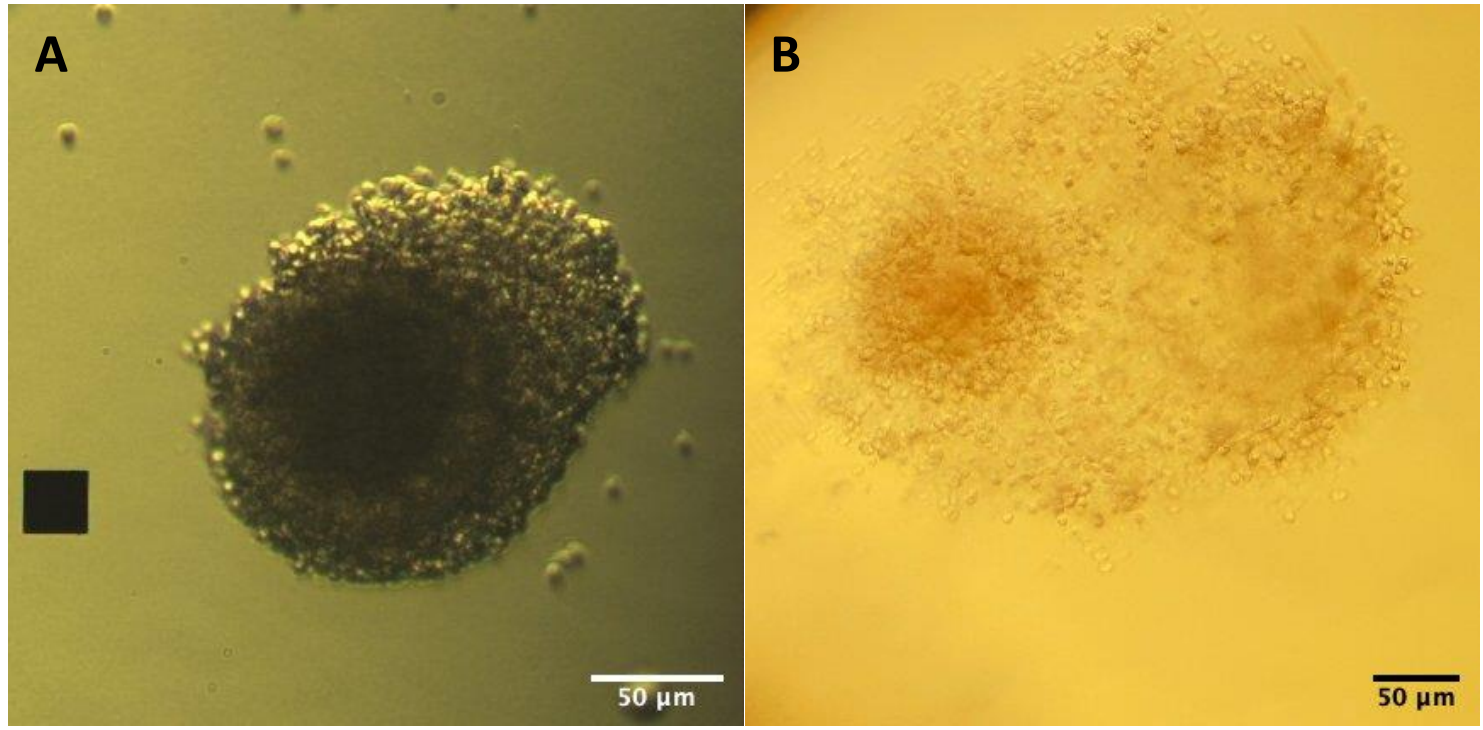

Figure 14: Non-expanded and expanded cumulus cells (CCs). [A]. Immature cumulus-cell oocyte complex (COC) with compact CC at time of collection. [B]. Mature COC with expanded CC at 24 hours. 


\subsection{Analyses of Metabolites in IVM Media}

The consumption and production of metabolites in the form of carbohydrates, amino acids and lipids are crucial to meet the energetic needs required for the progression and completion of cytoplasmic maturation thus, serve as ideal biomarkers to determine the completion of COC maturation and the attainment of developmental competency. However, the link between whether high or low levels of metabolic activity is beneficial or detrimental to embryo development has been heavily contested in literature (Leese, 2002; Gardner et al., 2011), similarly to the link between high mitochondrial activity during follicular development. A reason why metabolism and viability has been a challenge to quantify is due to the limitations of current technologies, such as metabolomics and radiolabelling, being unable to reproduce consistent results. These methods are generally expensive, time-consuming, invasive and require the sacrifice of embryos and COCs which also limits the robustness of data. Guerif et al. (2013) was able to produce a simple, non-invasive, high throughput consumption and release (CORE) method to analyse the metabolic activity of bovine embryos by determining the changes of glycolytic metabolite concentrations in culture media using refined ultramicrofluorometric techniques. More specifically, the consumption of glucose and pyruvate by the embryo was assumed as the loss of glucose and pyruvate in the media while the production of lactate was quantified by the increase of lactate in the media. This was quantified by utilising different enzymes involved within the glycolytic pathway where the catalysis of different metabolites and substrates was able to be detected through the process of autofluorescence.

However, the reported study assessed embryonic metabolism and, has yet to be utilised within an IVM system to analyse the metabolic activity for the COC. The COC is an interesting candidate for analysing metabolism as the oocyte itself is unable to utilise glucose and relies on the $\mathrm{CC}$ to convert glucose into other metabolic intermediates such as pyruvate which can be used as an energy source. The storage of pyruvate is also important for cleavage and blastocyst development (Brinster, 1969). Lactate production and presence in IVM media along with the presence of NAD has been reported to improve the quality of bovine oocytes in vitro (Cetica et al., 1999). 
For this study, we followed protocols based on methodology described by Guerif et al. (2013) and McKeegan (2015) to optimise and analyse the consumption of pyruvate by the COC within IVM media. Due to time constraint, we were unable to conduct optimisation studies for the consumption of glucose and production of lactate by the COC in IVM media. This will be further optimised in the Pitman lab in the future based on protocols reported by Guerif et al. (2013) and McKeegan (2015).

\subsubsection{Collection of IVM Media Samples}

IVM medium samples were collected at $1,7.5$ and 24 hours following GJ communication and GSH conjugation assays as described in sections 2.3 and 2.4 respectively. Samples were stored frozen at $-80^{\circ} \mathrm{C}$ until use. For control medium in which COCs had not been incubated, aliquots of each Early and Late IVM media for each treatment group was collected prior to the set-up of IVM plates and frozen at $-80^{\circ} \mathrm{C}$.

\subsubsection{Optimising for the Detection of Pyruvate in IVM Media}

To optimise detection of pyruvate in IVM media, minor alterations were made to the methodology described by Guerif et al. (2013) and McKeegan (2015). To accommodate for plate availability, flat-bottomed 96-well plates specific for fluorescence-based assays were used (Invitrogen, M33089) instead of the specified V-bottom 96-well plate. The flatbottom plate required a greater volume in each well in contrast to the V-bottom plate which is able to measure small volumes (i.e. $1 \mu \mathrm{L}$ sample to $10 \mu \mathrm{L}$ assay mix) utilised by by Guerif et al. (2013) and McKeegan (2015). Conforming to the 1:10 ratio, the volume was increased so that $6 \mu \mathrm{L}$ of sample was added to $60 \mu \mathrm{L}$ of the assay mix. The range of sodium pyruvate used to achieve a standard curve was also increased from 0 to $1 \mathrm{mM}$. A pyruvate reaction mix containing $0.1 \mathrm{mM} \mathrm{NADH}$ and $40 \mathrm{IU} / \mathrm{mL}$ lactate dehydrogenase supplemented in $4.6 \mathrm{mM}$ EPPS buffer ( $\mathrm{pH} 8.0$ ) was freshly prepared. To each well, $60 \mu \mathrm{L}$ of the assay mixture was loaded and background fluorescence was measured using a CLARIOStar Plus Microplate Reader (BMG Labtech) with excitation/emission of 340/460 $\mathrm{nm}$. Thereafter, $6 \mu \mathrm{L}$ of the sodium pyruvate standard was added to the assay mix (1:10 ratio) in triplicate and incubated in the dark at $37^{\circ} \mathrm{C}$ for 3 minutes. A reduction in fluorescence due to NADH oxidation was monitored. Standard curves with $R^{2}<0.98$ were 
rejected for optimisation studies. All samples and components of the assay were kept on ice unless specified.

\subsubsection{The Effect of Phenol Red on the Detection of Pyruvate Consumption}

Being a microultrafluorometric-based (MCB) assay, the effects of phenol-red in the commercial gold-standard IVM media (Gibco Life Technologies) on the fluorescent readings used to detect pyruvate consumption must be determined. Thus, two standard curves of sodium pyruvate were prepared in media with (Dulbecco's Modified Eagle's Medium, DMEM; Gibco Life Technologies) or without (phosphate-buffered saline, PBS) phenol red . Both sets of standards were run as described in Section 2.6.2. It is important to note that M199 was deliberately not utilised for standards as it contains glucose and sodium pyruvate. An M199 without sodium pyruvate is commercially available however, the absence of glucose is not due to it being a component of Earle's Salts.

As no significant differences in the level of pyruvate were measured in media in which phenol red was present (further discussed in section 3.4.1.1), from hereafter, a sodium pyruvate standard supplemented in phenol-red DMEM was utilised. This was considered to be more accurately representative of the IVM media.

\subsubsection{The Effect of Fresh vs. Freeze-thawed Assay Mix on Pyruvate}

\section{Consumption}

McKeegan (2015) stated that for embryo culture media, the assay mixture containing 0.1 $\mathrm{mM}$ NADH and 40IU/mL lactate dehydrogenase supplemented in $4.6 \mathrm{mM}$ EPPS buffer, $\mathrm{pH}$ 8.0 could be prepared prior to use for up to 3 months at $-20^{\circ} \mathrm{C}$. To confirm a similar relationship with IVM media, a freshly prepared and freeze-thawed assay mixture was plotted against a standard curve of 0-1 mM sodium pyruvate as described in Section 2.6.2.

\subsubsection{Detection of Pyruvate Consumption in IVM Medium}

For the following assays, a pyruvate reaction mix containing $0.1 \mathrm{mM} \mathrm{NADH}$ and $40 \mathrm{IU} / \mathrm{mL}$ lactate dehydrogenase supplemented in $4.6 \mathrm{mM}$ EPPS buffer, $\mathrm{pH} 8.0$ was freshly prepared. An aliquot of $60 \mu \mathrm{L}$ of assay mixture was loaded into each well of a 96-well plate 
and background fluorescence was measured using a CLARIOStar Plus Microplate Reader with excitation/emission of $340 / 460 \mathrm{~nm}$. An aliquot of $6 \mu \mathrm{L}$ of the sample medium (either the IVM media sample, the unincubated Early or Late control media samples or the pyruvate standard) was added to the assay mix at a 1:10 ratio and incubated in the dark at $37^{\circ} \mathrm{C}$ for 3 minutes. A reduction in fluorescence due to NADH oxidation was monitored. Final concentrations of pyruvate were determined against a six point standard curve (0-1 mM sodium pyruvate, Sigma Aldrich). To calculate the consumption rate, Early and Late media samples that had been collected prior to COC incubation was used for normalisation. The "Early" media was retained for the normalisation of 1 and 7.5 hour IVM samples whilst the "Late" media was retained for the normalisation of the 24 hour samples. Standard curves with $R^{2}$ values of $<0.99$ were rejected. All samples and components of the assay were kept on ice unless specified. A total of six samples collected from each treatment and timepoint were analysed. 


\subsection{Gene Expression Analyses}

\subsubsection{Sample Preparation}

Following completion of the incubation timepoints of 1, 7.5, 24 hours, the remaining two of the eight COCs that were not exposed to calcein-AM or further incubated in MCB were collected for gene expression analyses. These $\mathrm{COC}$ were placed into individual wells of a 96-well plate containing $20 \mu \mathrm{L}$ of pre-warmed PBS and denuded immediately. The CC mass of each $\mathrm{COC}$ was placed into individual $0.6 \mathrm{~mL}$ RNase-free Eppendorf tubes and pelleted by centrifugation at $800 \mathrm{~g}$ for 4 minutes. This pellet was washed with $20 \mu \mathrm{L}$ PBS and pelleted again via centrifugation. The supernatant was discarded and the cell pellet was snap-frozen on dry ice for 20 minutes before being transferred to a $-80^{\circ} \mathrm{C}$ freezer. Single denuded oocytes were placed into another $0.6 \mathrm{~mL}$ RNase-free Eppendorf tube, snapfrozen on dry ice for 20 minutes and stored at $-80^{\circ} \mathrm{C}$ until processing for RNA extraction.

\subsubsection{RNA Extraction}

Total RNA was extracted from single denuded oocytes and CC masses using the ArrayPure $^{\mathrm{TM}}$ Nano-scale RNA Purification Kit (Epicentre, United States) following the manufacturing protocol. All samples were centrifuged at $800 \mathrm{~g}$ briefly at $4^{\circ} \mathrm{C}$. For each sample, $0.1 \mu \mathrm{L}$ of $50 \mu \mathrm{g} / \mu \mathrm{L}$ Protein $\mathrm{K}$ was diluted into $30 \mu \mathrm{L}$ of $1 \mathrm{X}$ Nano-scale Lysis solution and mixed prior to being added to sample. The sample was then vortexed for 30 seconds and incubated at $67.5^{\circ} \mathrm{C}$ for 15 minutes. After this incubation period, each sample was placed on ice for 4 minutes. Thereafter, $18 \mu \mathrm{L}$ of MPC Protein Precipitation Reagent was added to the lysed sample and vortexed vigorously for 10 seconds. To pellet the debris, the sample was centrifuged for 7 minutes at $4^{\circ} \mathrm{C}$ and $12,000 \mathrm{~g}$ in a microcentrifuge. The resultant supernatant was transferred to a fresh $0.6 \mathrm{~mL}$ Eppendorf tube and precipitated by the addition of $50 \mu \mathrm{L}$ of isopropanol (Global Science, New Zealand) and the pellet was discarded. Following a 30 second vortex, the RNA was pelleted via centrifugation for 5 minutes at $4^{\circ} \mathrm{C}$ and $12,000 \mathrm{~g}$. The residual isopropanol was removed and the pellet airdried for 5 minutes. A DNase I solution was prepared by supplementing $40 \mu \mathrm{L}$ of $1 \mathrm{X}$ DNase buffer with $0.5 \mu \mathrm{L}$ of RNase-free DNase I. From this, $20 \mu \mathrm{L}$ was added to each sample, vortexed for 30 seconds and incubated at $37^{\circ} \mathrm{C}$ for 30 minutes to remove all contaminating DNA. After incubation, $20 \mu \mathrm{L}$ of $2 \mathrm{X}$ Nano-scale Lysis solution was added and the solution 
was vortexed for 5 seconds prior to the addition of $20 \mu \mathrm{L}$ of MPC Protein Precipitation Reagent. A further vortex step of 10 seconds was conducted before the sample was placed on ice for 4 minutes. The debris was then pelleted via centrifugation at $4^{\circ} \mathrm{C}$ for 5 minutes at $12,000 \mathrm{~g}$. The resultant supernatant containing RNA was transferred to a fresh $0.65 \mathrm{~mL}$ Eppendorf tube and the pellet discarded. An aliquot of $50 \mu \mathrm{L}$ of isopropanol was added to the sample and the RNA was pelleted by centrifugation at $4^{\circ} \mathrm{C}$ for 5 minutes at $12,000 \mathrm{~g}$. Without dislodging the RNA pellet, the isopropanol was aspirated. The pellet was then rinsed with $50 \mu \mathrm{L}$ of $70 \%$ ethanol and centrifuged for 3 minutes at $12,000 \mathrm{~g}$ at $4^{\circ} \mathrm{C}$. The residual ethanol was removed and the pellet was left to air-dry for 5 minutes. The pellet was then resuspended in $10 \mu \mathrm{L}$ of UltraPure ${ }^{\mathrm{TM}}$ DNase-free water (ThermoFisher Scientific, United States) and vortexed for 1 minute. To assist with RNA solubilisation, the RNA sample was incubated for 2 minutes at $55^{\circ} \mathrm{C}$, followed by a final 1 minute vortex and quick centrifugation. Thereafter, RNA samples were stored at $-80^{\circ} \mathrm{C}$ until cDNA synthesis.

\subsection{3. cDNA Synthesis}

Complementary DNA (cDNA) was synthesised from total RNA derived from single CC masses and denuded oocyte samples using the Superscript ${ }^{\text {TM }}$ VILO ${ }^{\text {TM }}$ CDNA Synthesis Kit (Invitrogen, ThermoFisher Scientific, United States) following the manufacturing guidelines. In a $0.2 \mathrm{~mL}$ PCR tube, $4 \mu \mathrm{L}$ of $5 X$ VILO reaction mix, $2 \mu \mathrm{L}$ of $10 \mathrm{X}$ Superscript enzyme mix, $4 \mu \mathrm{L}$ of UltraPure ${ }^{\mathrm{TM}}$ DNase-free water (Thermofisher Scientific, United States) and $10 \mu \mathrm{L}$ of the total RNA were added together and vortexed thoroughly. The cDNA synthesis step was performed on a Corbett Rotor-Gene ${ }^{\mathrm{TM}} 6000$ (Corbett Research Ltd, Australia) under the conditions of $25^{\circ} \mathrm{C}$ for 10 minutes, $42^{\circ} \mathrm{C}$ for 120 minutes and $85^{\circ} \mathrm{C}$ for 5 minutes. The CDNA samples were then stored at $-20^{\circ} \mathrm{C}$ until thawed for use in qPCR.

\subsubsection{Optimisation of Gene Biomarkers for Developmental Competency}

\subsubsection{Selection \& Design}

The genes chosen for the following experiments are established as biomarkers of developmental competency and maturation in ovine oocytes and CC which have been matured in vitro. These genes are namely, BMP15, GDF9, histone-induced factor 1-alpha (HIF1A), peroxiredoxin-6 (PRDX6), and superoxide dismutase 1 (SOD1) for oocytes; and 
connexin $43(C X 43)$, progesterone receptor $(P R)$, follicle-stimulating hormone receptor $(F S H R)$, epidermal growth factor receptor (EGFR), BCL2 apoptotic regulator (BCL2), BCL2 associated $X(B A X)$, glutathione-S-transferase alpha-1 (GSTA1), and tumour necrotic factor alpha induced protein 6 (TNFAIP6) for CC. Ribosomal lipoprotein 19 (RPL19) and peptidylprolyl isomerase A (PPIA) were used as reference genes and normalised for RNA input (Table 1; Table 4).

All accession numbers for gene candidates and the reference genes were derived from the National Center for Biotechnological Information (NCBI) gene database. All forward and reverse oligonucleotide primers, and Taqman $®$ probes used in this study were designed using the Premier Biosoft Beacon Designer Version 8 software package (Table 4). Primers and probes were designed by Sigma-Aldrich Proligo (Australia).

\subsubsection{Primer Optimisation}

To determine the optimal concentrations of each primer pair, cDNA from a pool of 20 untreated ovine COC was used as template. In some instances however, the expression levels of some of the CC-derived genes (i.e. BCL2, GSTA1, PR) were insufficient hence, pools of expanded COC matured within an IVM system were collected for optimisation.

Primer optimisation was conducted using reagents and protocols as described in the Agilent Brilliant II SYBR $®$ Green QPCR Master Mix kit. SYBR® Green is a dye which binds non-specifically to double-stranded DNA (dsDNA). During the denaturation step of the QPCR cycle, only a single-stranded cDNA template is present and thus, there is minimal fluorescence detected. However, as the annealing and extension steps occur, the synthesis of the dsDNA allowed for the SYBR $®$ Green intercalation and the emission of a fluorescent signal. The fluorescence is measured at the end of each annealing/extension step and is relative to the amount of dsDNA present. The cycle threshold $\left(C_{T}\right)$ is determined as the point at which the intensity of the fluorescent signal is significantly higher than background fluorescent levels and is dependent on the initial concentration of the template in the sample.

For each gene, a $2 \mathrm{X}$ reaction mix was prepared in $0.2 \mathrm{~mL} P C R$ tubes. The forward and reverse primer final concentrations were titrated from 50-500 nM. To $26 \mu \mathrm{L}$ of Brilliant II 
SYBR $®$ Green Master Mix, $2.08 \mu \mathrm{L}$ each of pooled cDNA template and primers were added and the final volume of $52 \mu \mathrm{L}$ was made up with UltraPure ${ }^{\mathrm{TM}}$ DNase-free water (ThermoFisher Scientific, United States). Following a brief vortex, duplicate aliquots of 25 $\mu \mathrm{L}$ were transferred to $0.1 \mathrm{~mL}$ strip tubes (Qiagen, Germany. Samples were amplified in a Corbett Rotor-Gene ${ }^{\mathrm{TM}} 6000$ (Corbett Research Ltd, Australia) under the following conditions: $1 \mathrm{cycle}$ at $95^{\circ} \mathrm{C}$ for 10 minutes, 40 cycles at $95^{\circ} \mathrm{C}$ for 15 seconds, $60^{\circ} \mathrm{C}$ for 60 seconds. The melt step, included to ensure that only a single product is produced, involved heating samples incrementally by $0.5^{\circ} \mathrm{C}$ for 5 seconds from $60^{\circ} \mathrm{C}$ to $95^{\circ} \mathrm{C}$. The most dilute concentration which yielded the lowest $C_{T}$ was chosen as the optimal primer concentration (Table 5). 
Table 4: Primers \& probes for genes selected for QPCR.

\begin{tabular}{|c|c|c|}
\hline Gene & NCBI accession number & Primer \& Probe Sequences \\
\hline \multirow[t]{3}{*}{ RPL19* } & \multirow[t]{3}{*}{ XM_004012836 } & F - TAAGCGAAAGGGTACTGCCAATG \\
\hline & & R - TTCTTAGATTCACGGTATCGTCTGAG \\
\hline & & P - [CY5]TTCTCATCCTCCTCATCCACGTTACCTTCTCGG[BHQ3] \\
\hline \multirow[t]{3}{*}{ PPIA* } & \multirow[t]{3}{*}{ NM_001308578 } & F - TTACTTAACCACCAGACCAT \\
\hline & & R - CGAGAGCACAAAGATTATAGG \\
\hline & & P - [6FAM]TCTGTAGCCCAGGAGAGCACCC[BHQ1] \\
\hline \multirow[t]{3}{*}{ BMP15 } & \multirow[t]{3}{*}{ NM_001114767 } & F - AGCTCTGGAATCACAAGG \\
\hline & & R - GTCCAATGATGAAGTGCC \\
\hline & & P - [ROX]ACCAGAACTCAAGAACCTCACTACCT[BHQ2] \\
\hline \multirow[t]{3}{*}{ GDF9 } & \multirow[t]{3}{*}{ NM_001142888 } & F-GACTCCTCAGTGCCAAGA \\
\hline & & R-AAGCGATTGAGCCATCAG \\
\hline & & P - [HEX]CATCCTGTGTACCTGCCAAGTATAGC[BHQ1] \\
\hline \multirow[t]{3}{*}{ HIF1A } & \multirow[t]{3}{*}{ XM_027971915 } & F-GCAAGAACCTCCTATAGC \\
\hline & & R - CGACTTCCAGTATCACTATA \\
\hline & & $\mathrm{P}-[$ [6FAM]CCACTACCACCGCCACCAAT[BHQ1] \\
\hline \multirow[t]{3}{*}{ PRDX6 } & \multirow[t]{3}{*}{ NM_001280704 } & F-AACGCTTACAATGGTGAA \\
\hline & & R - GGACAGTTTCAGTTTCTTATC \\
\hline & & $\mathrm{P}-[\mathrm{ROX}] \mathrm{ACACCACACGAGCAGTCACAG[BHQ2]}$ \\
\hline \multirow[t]{3}{*}{ SOD1 } & \multirow[t]{3}{*}{ NM_001145185 } & F-GAAGGTGATCATGGGTTC \\
\hline & & R - CCTCTCTTCATCCTTTGG \\
\hline & & P - [HEX]AGACAATACACAAGGCTGTACCAGTG[BHQ1] \\
\hline \multirow[t]{3}{*}{ CX43 } & \multirow[t]{3}{*}{ XM_004011159 } & F-TCTTCAAGTCTGTCTTCGAGGTG \\
\hline & & R - CTGATGCGGGCAGGGATC \\
\hline & & P -[HEX]CGGCACTCAAGCTGAATCCATAGATGTACCACT[BHQ1] \\
\hline \multirow[t]{3}{*}{ FSHR } & \multirow[t]{3}{*}{ NM_001009289 } & F- CAACCTGCTATACATCGA \\
\hline & & R-GTGCTTAATACCTGTGTTG \\
\hline & & P - [6FAM]CCTGATGCCTTCCAGAACCTTCC[BHQ1] \\
\hline \multirow[t]{3}{*}{$P R$} & \multirow[t]{3}{*}{ XM_015100878 } & F-CTTCCTAGAAGGACAGTTAC \\
\hline & & R - GACTCAAAGCTGTACTGTG \\
\hline & & P - [ROX]TCTGAATCGGGCCATCTCCTCT[BHQ2] \\
\hline \multirow[t]{3}{*}{$B A X$} & \multirow[t]{3}{*}{ XM_027978594 } & F-GACAGTAACATGGAGCTG \\
\hline & & R - GCTGGCAAAGTAGAAAAG \\
\hline & & $\mathrm{P}-[\mathrm{ROX}] \mathrm{CAGCCGTGGACACAGACTCTC[BHQ2]}$ \\
\hline \multirow[t]{3}{*}{ BCL2 } & \multirow[t]{3}{*}{ XM_027960877 } & F - CACAGGACTTCTGCAAATAC \\
\hline & & R - CCAGTTTGATTCCTAGACTTC \\
\hline & & P - [HEX]TAATTCATCCGCCGCCGCC[BHQ1] \\
\hline \multirow[t]{3}{*}{ EGFR } & XM_015102199 & F- GAGGAAATGTGCTTTATGAA \\
\hline & & R - AGGGTTGTTACTGAATCG \\
\hline & & P - [6FAM]ATGCCTTAGCCGTCTTATCCAACTATG[BHQ1] \\
\hline GSTA1 & XM_027958329 & F-TCCCTGCATTTGAAAATG \\
\hline & & R - TCCACATAGTAGAGAAGTTC \\
\hline & & P - [HEX]AGAGCCACGGACAAGACTACCT[BHQ1] \\
\hline TNFAIP6 & XM_004004704 & F-ATGGGGATTCAAGAATGG \\
\hline & & R-ACTGCCACCTTCATATTC \\
\hline & & $\mathrm{P}-[$ [6FAM]CACCGCCTTCGCTTCTGC[BHQ1] \\
\hline
\end{tabular}

* Reference genes 


\subsubsection{TaqMan $®$ Probe Optimisation}

The multiplex reactions used in this study applied TaqMan $®$ chemistry which utilises specific fluorophore and quencher combinations. When the fluorophore and quencher are in close proximity, there is no detectable fluorescence as the probe has not yet hybridised. A fluorescent signal is emitted during the annealing-extension step in which the Taq polymerase separates the quencher from the fluorophore.

In similar fashion to the primers, the optimal probe concentration was determined by curating a $2 \mathrm{X}$ reaction mix with titrations of the appropriate probe from a concentration range of 50-500 nM. Thus in addition to $26 \mu \mathrm{L}$ of Brilliant Multiplex QPCR Master Mix, each of forward and reverse primers at optimal concentrations, and $2.08 \mu \mathrm{L}$ of pooled template were made up to a final volume of $52 \mu \mathrm{L}$ corrected with UltraPure ${ }^{\mathrm{TM}}$ DNase-free water. Following a brief vortex, aliquots of $25 \mu \mathrm{L}$ were transferred to $0.1 \mathrm{~mL}$ strip tubes (Qiagen, Germany) in duplicate. Samples were amplified in the a Corbett Rotor-Gene ${ }^{\mathrm{TM}}$ 6000 (Corbett Research Ltd, Australia) under the following conditions: 1 cycle at $95^{\circ} \mathrm{C}$ for 10 minutes, 40 cycles at $95^{\circ} \mathrm{C}$ for 15 seconds, $60^{\circ} \mathrm{C}$ for 60 seconds. The most dilute concentration which yielded the lowest $C_{T}$ was chosen as the optimal probe concentration (Table 5).

\subsubsection{Multiplex Optimisation}

To ensure that multiplex reactions were optimised, the reaction efficiency of each gene in the multiplex sets was determined (Table 6). In each case, $2.08 \mu \mathrm{L}$ of a serially-diluted (1:1 to 1:256) template consisting of 200 pooled COC (100 non-expanded and 100 expanded) was prepared. For each multiplex set, a $2 X$ reaction mix containing $26 \mu \mathrm{L}$ Brilliant Multiplex QPCR Reaction Mix, optimised concentrations of the appropriate primer and probe (Table 5) and $2.08 \mu \mathrm{L}$ of the serially-diluted template was added to a $0.2 \mathrm{~mL}$ PCR tube and corrected to a total volume of $52 \mu \mathrm{L}$ with UltraPure ${ }^{\mathrm{TM}}$ DNase-free water. Following a brief vortex, aliquots of $25 \mu \mathrm{L}$ were transferred to $0.1 \mathrm{~mL}$ strip tubes (Qiagen, Germany) in duplicate. Samples were amplified in a Corbett Rotor-Gene ${ }^{\mathrm{TM}} 6000$ (Corbett Research Ltd, Australia) under the following conditions: 1 cycle at $95^{\circ} \mathrm{C}$ for 10 minutes, 40 cycles at $95^{\circ} \mathrm{C}$ for 15 seconds, $60^{\circ} \mathrm{C}$ for 60 seconds. 
The $C_{T}$ should increase by a value of 1 after each dilution as the cDNA template is being halved each time. This relationship allows for the reaction efficiency to be determined by the assessment of linear regression, standard curve and $R^{2}$ values. The Rotor-Gene ${ }^{\mathrm{TM}} \mathrm{Q}$ Series Software allows one to enter standard arbitrary values of halving concentrations as a standard curve which can automatically output a reaction efficiency value and graph for each gene within the multiplexed set. For multiplex reactions, only efficiencies between $80-103 \%$ with an $\mathrm{R}^{2}>0.98$ were regarded.

While some sets (Oocyte Set 1, CC Set 1, CC Set 3) had been previously optimised in the Pitman Lab (Clark, 2018), the reaction efficiencies were repeated to check for primer and probe degradation.

Table 5: Optimised concentrations for primers \& probes for QPCR.

\begin{tabular}{lccc}
\hline & Forward primer (nM) & Reverse primer (nM) & Probe (nM) \\
\hline RPL19* & 200 & 200 & 50 \\
\hline PPIA* & 300 & 300 & 50 \\
\hline BMP15 & 400 & 300 & 100 \\
\hline GDF9 & 200 & 300 & 150 \\
\hline HIF1A & 400 & 300 & 100 \\
\hline PRDX6 & 300 & 200 & 100 \\
\hline SOD1 & 100 & 200 & 50 \\
\hline CX43 & 200 & 50 & 50 \\
\hline FSHR & 300 & 200 & 50 \\
\hline PR & 400 & 500 & 100 \\
\hline BAX & 200 & 300 & 50 \\
\hline BCL2 & 200 & 200 & 50 \\
\hline EGFR & 300 & 300 & 50 \\
\hline GSTA1 & 500 & 400 & 100 \\
\hline TNFAIP6 & 500 & 400 & 50 \\
\hline Reference & & & 50 \\
\hline
\end{tabular}

* Reference genes 
Table 6: Multiplex reaction efficiencies.

\begin{tabular}{|c|c|c|c|}
\hline Multiplex Set & Gene & $\mathbf{R}^{2}$ & Efficiency (\%) \\
\hline \multicolumn{4}{|l|}{ OOCYTE } \\
\hline \multirow[t]{4}{*}{ Multiplex Set 1* } & RPL19 & 0.98 & $89 \%$ \\
\hline & $B M P 15$ & 0.98 & $84 \%$ \\
\hline & GDF9 & 0.98 & $82 \%$ \\
\hline & PPIA & 0.98 & $88 \%$ \\
\hline \multirow[t]{4}{*}{ Multiplex Set 2} & RPL19 & 0.99 & $103 \%$ \\
\hline & HIF1A & 0.99 & $81 \%$ \\
\hline & SOD1 & 0.99 & $96 \%$ \\
\hline & PRDX6 & 0.99 & $82 \%$ \\
\hline \multicolumn{4}{|l|}{ CUMULUS CELL } \\
\hline \multirow[t]{2}{*}{ Multiplex Set 1* } & RPL19 & 0.99 & $93 \%$ \\
\hline & PPIA & 0.99 & $85 \%$ \\
\hline \multirow[t]{4}{*}{ Multiplex Set 2} & RPL19 & 0.99 & $84 \%$ \\
\hline & $B A X$ & 0.99 & $84 \%$ \\
\hline & $B C L 2$ & 0.98 & $100 \%$ \\
\hline & $E G F R$ & 0.98 & $80 \%$ \\
\hline \multirow[t]{4}{*}{ Multiplex Set 3* } & RPL19 & 0.99 & $97 \%$ \\
\hline & $C X 43$ & 0.99 & $85 \%$ \\
\hline & FSHR & 0.98 & $83 \%$ \\
\hline & $P R$ & 0.98 & $81 \%$ \\
\hline \multirow[t]{3}{*}{ Multiplex Set 4} & $R P L 19$ & 0.99 & $96 \%$ \\
\hline & GSTA1 & 0.99 & $97 \%$ \\
\hline & TNFAIPG & 0.98 & $80 \%$ \\
\hline
\end{tabular}

* Designed by Clark (2018) 


\subsubsection{Quantitative PCR Protocol}

The relative expression levels of the candidate genes were measured in the experimental samples. A calibrator template consisting of $200 \mathrm{COC}$ in a expanded $(N=100)$ and nonexpanded $(N=100)$ state were included as a standard for all gene sets and technical replicates. Each run also incorporated a no reverse transcriptase and a no template control to check for genomic or cDNA contamination, respectively.

A $2 X$ reaction mix containing $26 \mu \mathrm{L}$ Brilliant Multiple QPCR Reaction Mix, the optimal concentrations of the appropriate primer and probe and $2.08 \mu \mathrm{L}$ neat template was added to a $0.2 \mathrm{~mL}$ PCR tube and corrected to a total volume of $52 \mu \mathrm{L}$ with UltraPure ${ }^{\mathrm{TM}}$ DNasefree water. For each reaction run, multiple samples were included (e.g. 36 samples in a Corbett Rotor-Gene ${ }^{\mathrm{TM}} 6000$ 72-ring disc in duplicate), and so a 36X reaction mix, excluding the cDNA template, was prepared of which $49.92 \mu \mathrm{L}$ of this reaction mix would be aliquoted into a $0.2 \mathrm{~mL}$ PCR tube and $2.08 \mu \mathrm{L}$ template added for a total volume of $52 \mu \mathrm{L}$. Following a brief vortex, aliquots of $25 \mu \mathrm{L}$ were transferred to $0.1 \mathrm{~mL}$ strip tubes (Qiagen, Germany) in duplicate. Samples were amplified in the a Corbett Rotor-Gene ${ }^{\mathrm{TM}} 6000$ (Corbett Research Ltd, Australia) under the following conditions: 1 cycle at $95^{\circ} \mathrm{C}$ for 10 minutes, 40 cycles at $95^{\circ} \mathrm{C}$ for 15 seconds, $60^{\circ} \mathrm{C}$ for 60 seconds. The amplification results were analysed on the Qiagen Rotor-Gene Q Software version 1.7. 


\subsection{Granulosa Cell Proliferation Assay}

Granulosa cells (GCs) play a major role in maturation of the oocyte during follicular development through various molecular signals. Their function and proliferation is known to be heavily mediated by the secretion of OSFs, and in particular that of GDF9 and BMP15. To determine the effects of ratios of GDF9:BMP15 $(1: 6,1: 1,6: 1)$ on the proliferation of ovine $\mathrm{GC}$, a ${ }^{3} \mathrm{H}$-thymidine incorporation bioassay was conducted using methods described by Heath (2016) and Christoforou (2017).

\subsubsection{Preparation of Granulosa Cell Incubation Media}

A GC incubation media consisting of $50 \mathrm{~mL}$ McCoys 5 a medium with sodium bicarbonate (Gibco Life Technologies, 16600-082) containing $0.3 \mathrm{mg} / \mathrm{mL}$ polyvinyl alcohol (PVA) and $100 \mathrm{IU} / \mathrm{mL}$ penicillin-streptomycin, was prepared and filtered into a $50 \mathrm{~mL}$ cell culture flask. This was equilibrated in an incubator at $39^{\circ} \mathrm{C}, 5 \% \mathrm{CO}_{2}$ and $100 \%$ humidity for at least 4 hours prior to use.

\subsubsection{Preparation of Treatments}

As described in Section 2.2.3 above, ratios of GDF9, BMP15 and an empty vector control were prepared based on mRNA expression levels within oocytes of mammals of varying litter sizes (Table 7). This was prepared using in-house HEK-293 cell secreted media containing recombinant pGDF9 and pBMP15. The concentration of pGDF9 and pBMP15 within the secreted media batch used in this thesis were $0.94 \mathrm{ng} / \mu \mathrm{L}$ and $0.58 \mathrm{ng} / \mu \mathrm{L}$, respectively (Alhussini, 2016; Swinerd, 2016). These preparations are un-purified, hence it is important to note that these preparations may contain contaminants from the HEK293 cells. To mitigate any possible effects from the HEK-293 contaminants, the control GCs received the same volume of secreted media from HEK-293 transfected with the empty vector, as the treated GC. In additional to these treatments, a positive control of $550 \mathrm{ng} / \mathrm{mL}$ activin A and a negative control of media alone were also included. Activins are dimeric glycoprotein from the TGF- $\beta$ superfamily of proteins, that are primarily produced by GCs. Activins are known positive regulators of FSH biosynthesis and secretion, as well as mediators that enhance the growth of the ovarian follicle and 
promote GC proliferation (Zhang et al., 2013). Unfortunately, while activin A was initially included as a positive control, it did not increase the proliferation rate significantly. Given the large body of evidence for GDF9 and BMP15 as mitogenic activators of ovine GC, the treatments acted as their own positive controls. Treatments were prepared $6 \mathrm{X}$ to add to multiple wells.

Table 7: Treatment preparation summary for granulosa cell proliferation assay. The volumes $(\mu \mathrm{L})$ of HEK293 cell secretions containing porcine (p) GDF9, BMP15 or empty vector (EV) added to a total volume of $55 \mu \mathrm{L}$ for each treatment preparation. To mitigate any effects of HEK293 expressed media, an EV secreted media was used to ensure the same volume was added to each $55 \mu \mathrm{L}$ preparation.

\begin{tabular}{|c|c|c|c|c|c|}
\hline & \multirow[b]{2}{*}{ Activin A } & \multirow[b]{2}{*}{ Control } & \multicolumn{3}{|c|}{ GDF9:BMP15 Ratio } \\
\hline & & & 1:6 & $1: 1$ & $6: 1$ \\
\hline $\begin{array}{l}\text { pGDF9 } \\
(0.94 \mathrm{ng} / \mu \mathrm{L})\end{array}$ & & & 1.0 & 1.0 & 5.9 \\
\hline $\begin{array}{l}\text { pBMP15 } \\
(0.58 \mathrm{ng} / \mu L)\end{array}$ & & & 9.5 & 1.6 & 1.6 \\
\hline EV & 9.5 & 10.5 & & 7.9 & 3.0 \\
\hline Activin A & & & & & \\
\hline$(0.55 \mathrm{ng} / \mu \mathrm{L})$ & 1.0 & & & & \\
\hline $\begin{array}{l}\text { Cell suspension in } \\
\text { media }\end{array}$ & 44.5 & 44.5 & 44.5 & 44.5 & 44.5 \\
\hline Total vol/well ( $\mu \mathrm{L})$ & 55.0 & 55.0 & 55.0 & 55.0 & 55.0 \\
\hline
\end{tabular}

\subsubsection{Granulosa Cell Collection \& Preparation}

Primary cells were extracted from 1-5 mm visible follicles using the dissection method for COC extraction described in Section 2.2.4. The COCs and denuded oocytes were removed, pooled and processed for calibrators used in QPCR as described in section 2.7. The remaining cells which would consist of a mixed population were presumed to be mostly GC. These cells were aspirated twice with a 20-gauge needle in order to disperse any clumps. The cells were then collated into a $15 \mathrm{~mL}$ falcon tube and centrifuged at $450 \mathrm{~g}$ for 
5 minutes at $20^{\circ} \mathrm{C}$. The supernatant was removed carefully without disrupting the pellet. Cells were gently washed and centrifuged twice in $5 \mathrm{~mL} \mathrm{GC}$ incubation media consisting of McCoy's 5A (Gibco Life Technologies, USA) supplemented with $0.3 \mathrm{mg} / \mathrm{mL}$ PVA (Sigma Aldrich, USA) and $100 \mathrm{lU} / \mathrm{mL}$ Penicillin-Streptomycin (Gibco Life Technologies, USA) (Appendix 2). The supernatant was carefully removed and discarded after each wash step. The pelleted cells were then gently resuspended in $1 \mathrm{~mL}$ of GC incubation media, ensuring no clumps were visible.

Thereafter, a $50 \mu \mathrm{L}$ aliquot of cells was removed and mixed with $5 \mu \mathrm{L}$ of $0.4 \%$ trypan blue (Gibco Life Technologies, USA). An aliquot of $10 \mu \mathrm{L}$ was pipetted under the coverslip on both chambers of a haemocytometer with a $0.1 \mathrm{~mm}$ depth (Improved Neubauer, BS 74B Hawksley). Trypan blue is a dye which is excluded by viable cells with intact plasma membranes. Under a Leica DM1000 microscope, the number of live (clear) and dead (blue) cells with GC morphology were counted and recorded (Figure 15). GC have a distinct morphological appearance consisting of a large, round size exhibiting a granular cytoplasm and prominent nucleus. Once counted, the numbers and proportion of viable GC were used to determine live GC concentrations using the equation below. This calculation was then used to further dilute cells so that 20,000 live cells were present per $44.5 \mu \mathrm{L}$ cell suspension added to each well.

\section{Concentration $($ cells $/ \mathrm{mL})=$ \# live cells $\times 10^{4} \times$ dilution factor $\mathrm{x}$ volume $\%$ viability = \# live cells / (\# live cells + \# dead cells)}

\subsubsection{Granulosa Cell Incubation}

The following experiments were conducted in delta-treated 96-well plates (NUNC, Thermo Fisher). In five wells of the first five rows, 20,000 viable GC in $44.5 \mu \mathrm{L}$ was added. This was followed by $10.5 \mu \mathrm{L}$ of the empty vector control, 1:6, 1:1, 6:1 GDF9:BMP15, Activin A (if included) to the wells of the first, second, third, fourth and fifth row, respectively. To the sixth row, $55 \mu \mathrm{L}$ of incubation media without cells was added directly to five wells. To prevent excessive dehydration of the wells, all empty wells within the plate were loaded with $65 \mu \mathrm{L}$ of media (phenol red-free M199; Gibco Life Technologies, 
USA). The plate was shaken gently and then placed into the incubator at $39^{\circ} \mathrm{C}, 5 \% \mathrm{CO}_{2}$ and $100 \%$ humidity for 16 hours.

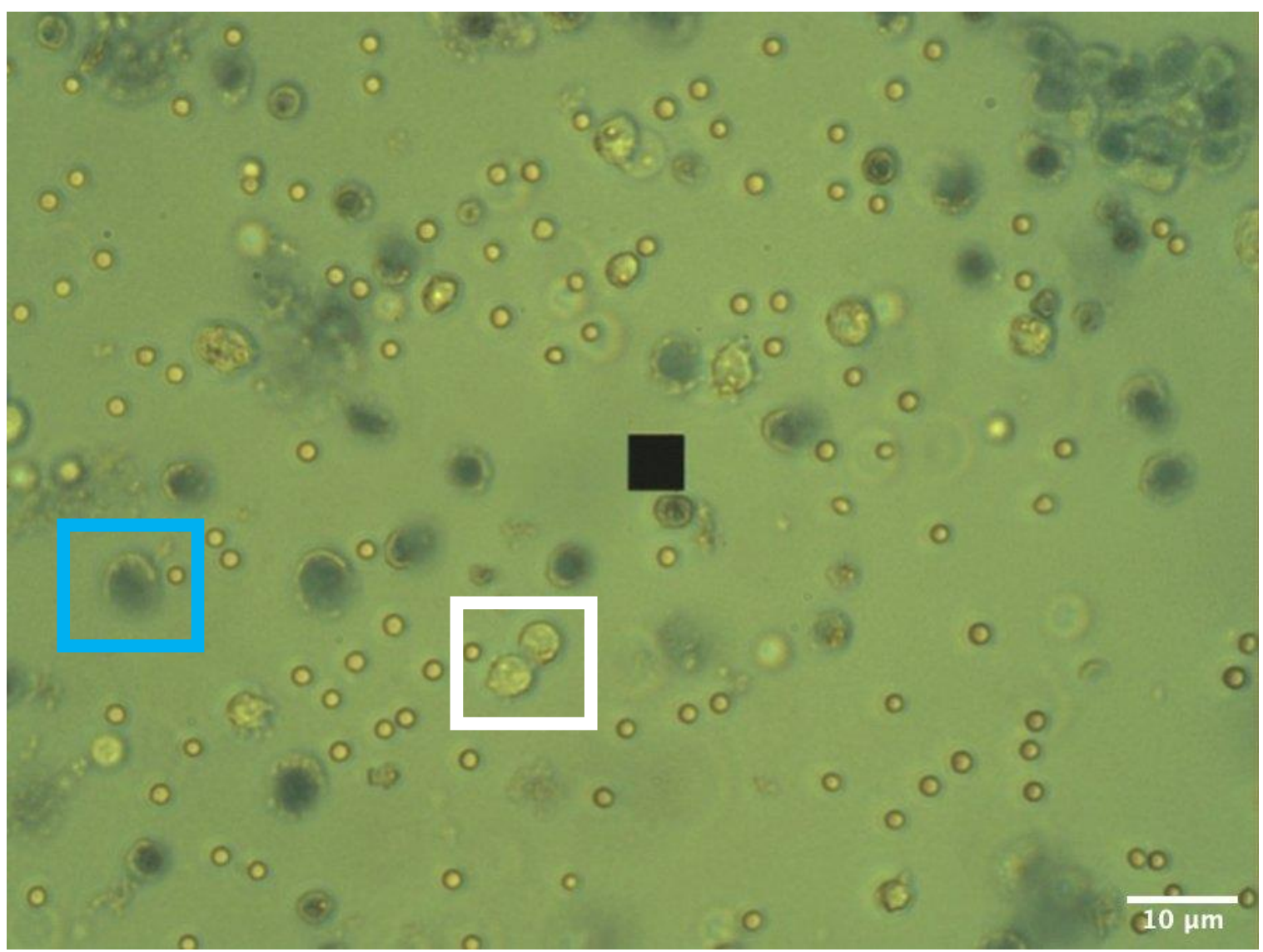

Figure 15: Granulosa Cell (GC) morphology. Live GC (depicted as large, clear and granulated cytoplasm) and dead GC (depicted as large, blue cells with a clear membrane) were stained by trypan blue and assessed for $\%$ viability.

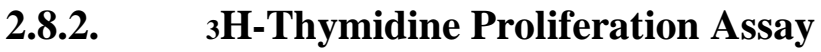

Following the stipulated incubation period, plates were removed from the incubator for the radiolabelling by tritiated $\left({ }^{3} \mathrm{H}\right)$ thymidine (Perkin Elmer, USA) which incorporates itself into proliferating cells. The tritiated thymidine was diluted 1:25-fold in the GC incubation media. Thus $10 \mu \mathrm{L}$ containing $\sim 0.4 \mu \mathrm{Ci}$ was added to each treatment and control well, resulting in a final volume of $65 \mu \mathrm{L}$. The plate was returned to the incubator for a further 6 hours.

Thereafter, the plate was removed for cell harvesting. Cells were transferred onto a glass fibre filter mat (1450 micro $\beta$ fibre filter, 90×120mm printed filter mat, Perkin Elmer, MA, 
USA) using the 'Pulse Wash' function on a Wallac Cell Harvester (TOMTEC). The filter mat was then placed into a $1500 \mathrm{~W}$ microwave oven for 1 minute (or until dry) and transferred to a sample bag so that the filter mat was flush to the edge of the bag (Perkin Elmer, MA, USA). The outer edges of the bag were heat-sealed and one corner of the bag was cut. A $4.5 \mathrm{~mL}$ aliquot of $\beta$-scintillation fluid was added through the cut until the fluid was evenly dispersed over the filter mat. Excess scintillation fluid was carefully removed. The edge of the bag was heat sealed flush to the edges of the filter mat. All excess edges of the bag containing the mat were trimmed so that it could fit into a scintillation counter cassette. The cassette was then placed into the Wallac micro $\beta$ trillux scintillation counter (1450 LSC and Luminescence counter, Perkin Elmer) and $\beta$-particle emissions at each position of the filter mat were counted for 2 minutes and recorded as counts per minute (CPM). 


\subsection{Statistical Analyses}

All statistical analyses were performed using IBM SPSS $®$ analytical software. Graphs were produced using GraphPad Prism Version 8. All significant differences were established at a significance level of $P<0.05$. (* $P<0.05 ;{ }^{* *} P<0.01 ; * * * P<0.001 ; * * * P<0.0001$ )

\subsubsection{Gap Junction and Glutathione Assays}

For GJ communication and intra-oocyte GSH assays, individual multiple comparison twoway ANOVAs were conducted, followed by Bonferroni and Tukey HSD post-hoc tests to further assess significant effects. Raw data was collated and the mean fluorescence values are displayed as means \pm SEM. To determine the relative fluorescence, the raw fluorescence values of all oocytes incubated in the IVM system for all treatments for each replicate were corrected against the mean value of $\mathrm{COC}$ from the 1 hour timepoint. The resultant relative fluorescence values are displayed as means \pm SEM. For both raw data and normalised data, the Levene test for normality was utilised. For both sets of data, log transformations were required for normal distribution. Data for $\mathrm{COC}$ with $\mathrm{CC}$ of grades 4 and 5 (Table 3) were excluded from the dataset as they do not truly demonstrate effects of the assays due a higher chance of direct penetration and diffusion of probes through the oocyte.

\subsubsection{Morphological Assessment}

For both GVBD and CC grade data (Grade 1-3; Table 3), contingency tables and chi-squared analyses interrogating observed and expected values collected from IVM studies (GJ and GSH assays) were used to determine significant differences between treatments and incubation endpoints.

\subsubsection{Pyruvate Consumption Assays}

Samples collected at 1-7.5 hours incubation were corrected against the Early control media of its associated treatment group and changes in concentration of pyruvate in media samples over the incubation period were determined against a standard curve of sodium pyruvate. The resultant values were negative, representing loss of pyruvate from 
media due to pyruvate consumption by the COC. To show the rate of pyruvate consumption by $\mathrm{COC}$, data was inversed to demonstrate a positive relationship between rate of consumption over the incubation period. The values are displayed as means \pm SEM. A Levene's test for normality was utilised for the data. Individual multiple comparison two-way ANOVAs were conducted followed by Bonferroni and Tukey HSD post-hoc tests to further assess significant effects. Optimisation studies were analysed via linear regression to determine differences between media and assay mixture conditions.

\subsubsection{Gene Expression}

For gene expression analyses, all CC and oocyte samples were run in duplicates in two separate technical replicate runs, and normalised against expression levels of the reference gene RPL19. This essentially corrects for variations in loading of CDNA in each reaction. To account for between-assay variation, a calibrator sample was included in every run. To determine the fold-change in expression of candidate genes, $2^{\text {(- } \Delta \Delta C T)}$ was employed. To do this, cycle threshold $\left(C_{T}\right)$ for each query gene was normalised against the $\mathrm{C}_{\mathrm{T}}$ of RPL19 in order to obtain a $\triangle \mathrm{CT}$ value. This was then compared against the average normalised $C_{T}$ value of the calibrator to obtain $2^{(-\Delta \Delta C T)}$. The mean $\pm S E M 2^{(-\Delta \Delta C T)}$ value was calculated from the technical replicates for oocytes and CC collected from 1, 7.5 hour and 24 hour incubations alongside the SEM. All data was tested for normality by performing a P-P Plot. All data that were not normal distributed were transformed using either natural log or square-root transformations. A two-way ANOVA was then performed on the data, followed by a Bonferroni post-hoc test to assess significance.

\subsubsection{Granulosa Cell Proliferation Assay}

Raw proliferation values and data were normalised against the control treatment. The values are displayed as means \pm SEM. A non-parametric Kruskill-Wallis was conducted against both raw and normalised data. 


\section{CHAPTER 3: RESULTS}

\subsection{Gap Junction Communication Assays}

\subsubsection{Optimisation for Timepoints}

The intra-oocyte fluorescence levels of COCs incubated in IVM media with no OSF supplementation for $1,4,7.5,16,20$ and 24 hours, as an indirect assessment of GJ communication, are depicted in Figure 16. Overall, there was no significant difference in GJ activity over the incubation period.

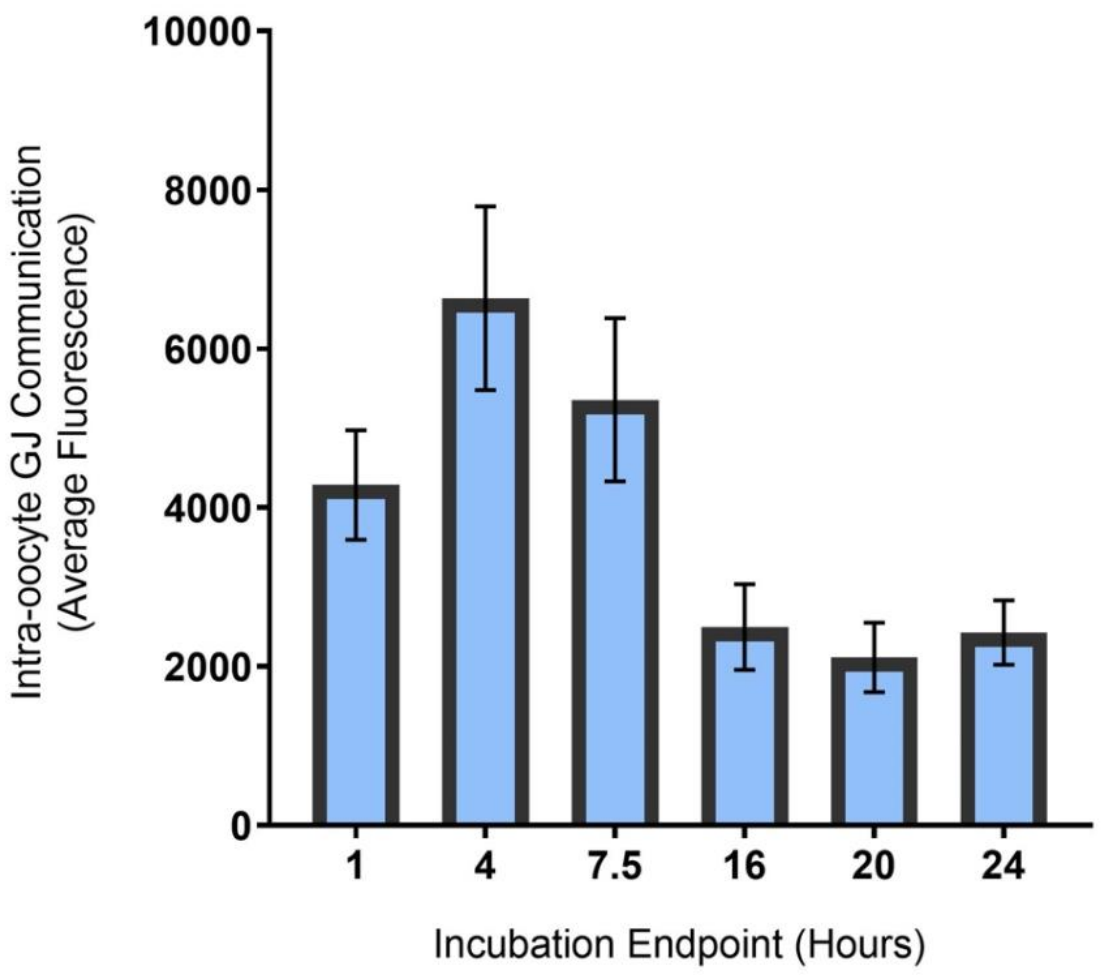

Figure 16: Effect of time on gap junction (GJ) communication in ovine $\mathrm{COC}$ in a biphasic IVM culture system. Intra-oocyte fluorescence levels in ovine COC were measured after $1,4,7.5,12,20$ and 24 hours in a control biphasic IVM system. Data shown as mean \pm SEM fluorescent levels from four replicate experiments containing eight COC. Two-way ANOVA demonstrates no significant difference in fluorescent levels between timepoints. 
To account for variability due to seasonality and media preparation between the four biological replicate experiments, data from the 4, 7.5, 16, 20 and 24 hour timepoints were normalised against the data at 1 hours for each replicate (Figure 17). Normalisation of the data in this manner demonstrated a significant difference $(P<0.002)$ in the fold-change in intra-oocyte fluorescence with time in culture. Specifically, GJ communication was highest at 4 hours and lower $(P<0.002)$ at all timepoints from 16 hours onwards.

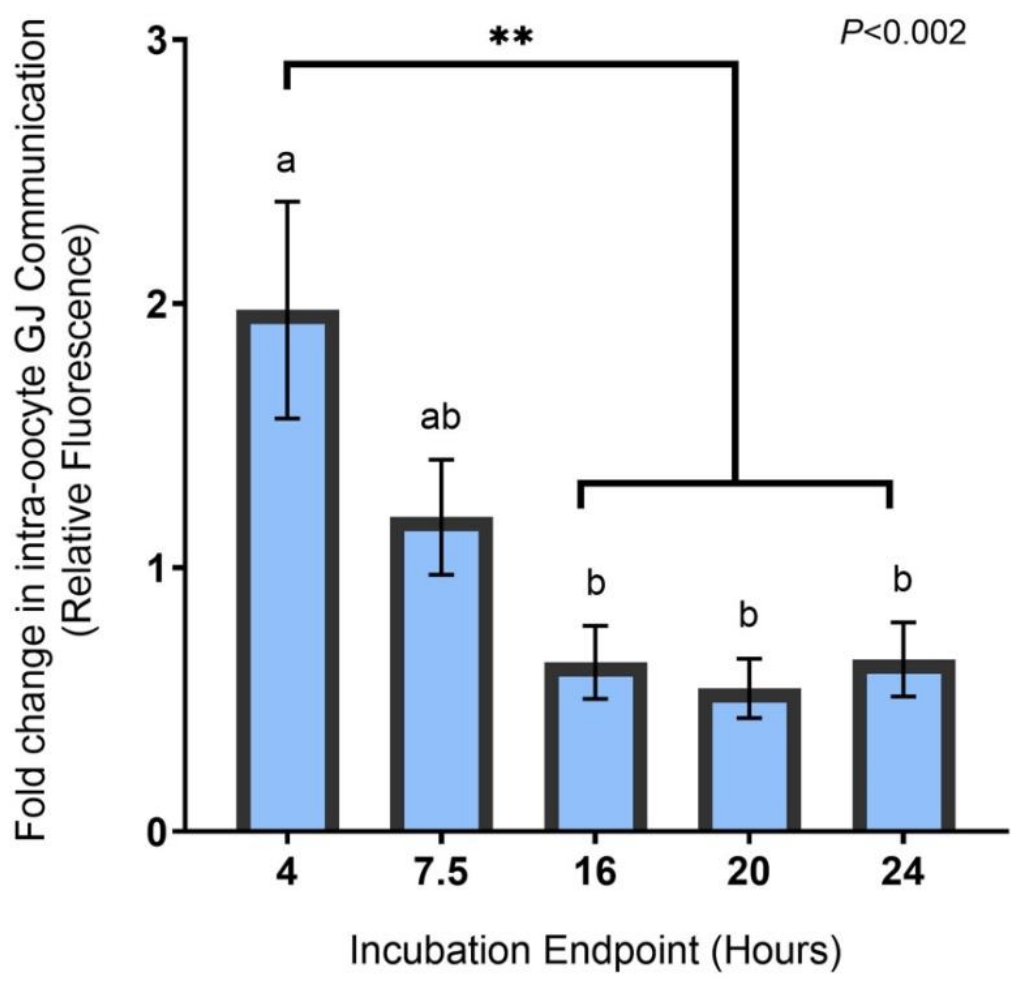

Figure 17: Fold change of gap junction (GJ) communication, represented by intra-oocyte fluorescence levels, in ovine COC incubated in an IVM culture system over $\mathbf{2 4}$ hours. Data were normalised to the 1 hour timepoint. Data shown as mean \pm SEM fluorescent levels for replicate experiments involving eight COC. Significant differences at $P<0.002$ are denoted by different alphabetical superscripts $(a, b)$ and asterisks $(* *)$. 


\subsubsection{Experimental Gap Junction Communication}

The intra-oocyte florescence levels in ovine $\operatorname{COC}$ at 1, 7.5, 24 hours of incubation in a goldstandard IVM system supplemented with different ratios of GDF9:BMP15 or an empty vector control (no GDF9:BMP15) were measured as an indirect assessment of GJ communication. The results are depicted in Figure 18. Overall, there was a significant difference in intra-oocyte fluorescence levels with time in culture, where GJ communication was lower after 24 hours, relative to 1 hour $(P<0.001)$.

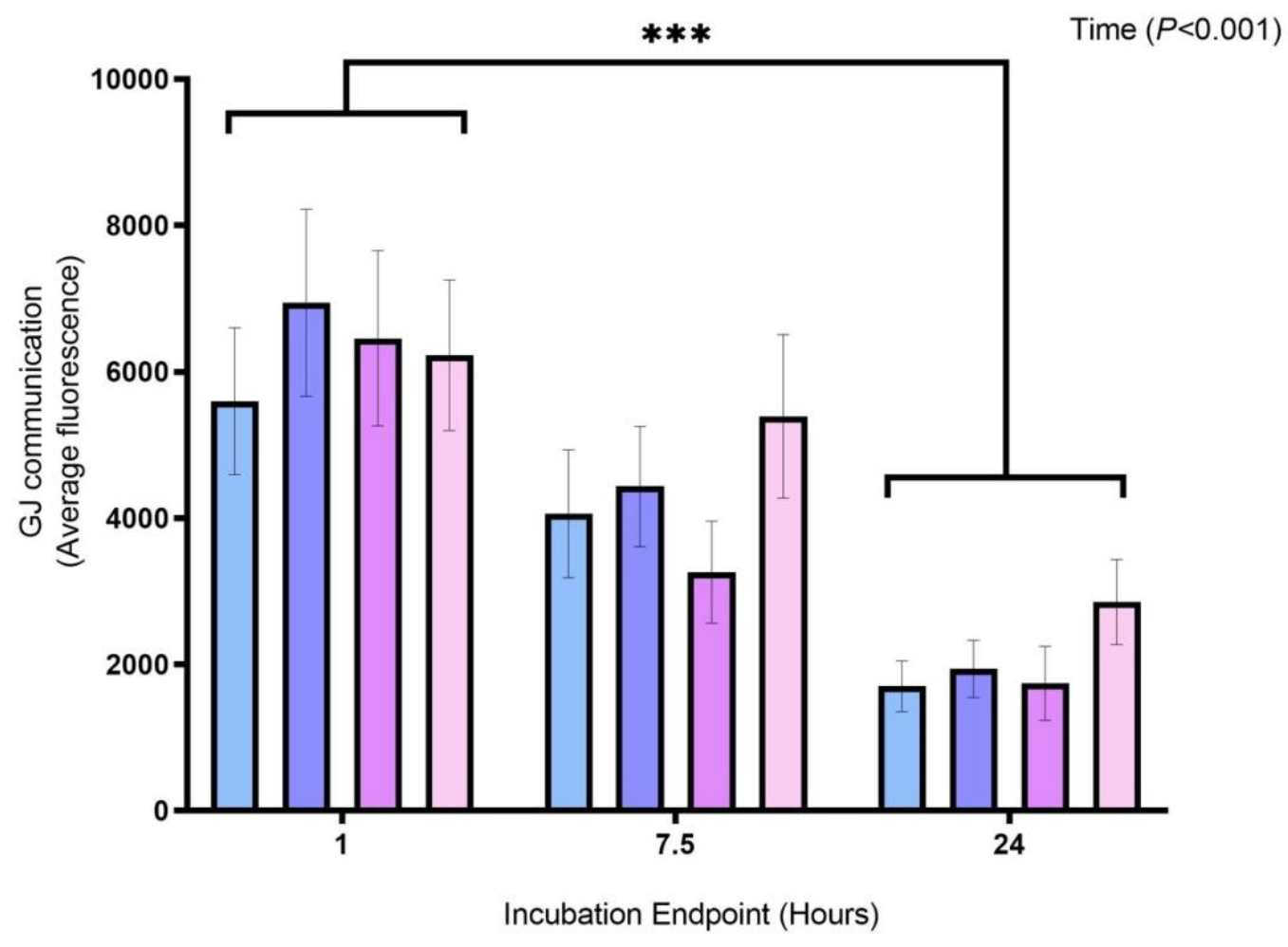

Figure 18: Effects of different ratios of GDF9 and BMP15 in a biphasic IVM culture system on gap junction (GJ) communication in ovine COC. Intra-oocyte fluorescent levels in ovine COC after 1, 7.5 and 24 hours of incubation in a biphasic IVM media system supplemented with differing ratios of GDF9:BMP15. Control group (no GDF9:BMP15) [ $\square$ ], and

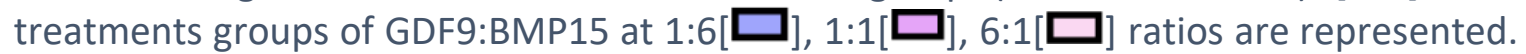
Raw data are shown as mean \pm SEM fluorescent levels from four replicate experiments involving six $\mathrm{COC}$ per treatment group. Two-way ANOVA demonstrates significant differences in time $(P<0.001)$ between relative fluorescence and incubation times denoted by $(* * *)$. 
To account for variability due to seasonality and media preparations between the four biological replicate experiments, data at 7.5 and 24 hours of incubation was again normalised against that at 1 hours for each replicate (Figure 19). Normalisation of the data in this manner demonstrated a treatment difference $(P=0.022)$ in intra-oocyte fluorescence levels. Specifically, GJ communication was higher in the 6:1 GDF9:BMP15 ratio after 24 hours in culture, when compared to the lower ratios $(1: 6 ; P=0.025,1: 1$; $P=0.026)$ and a trend in regards to the control $(P=0.092)$. There were no treatment differences observed at 7.5 hours of incubation.

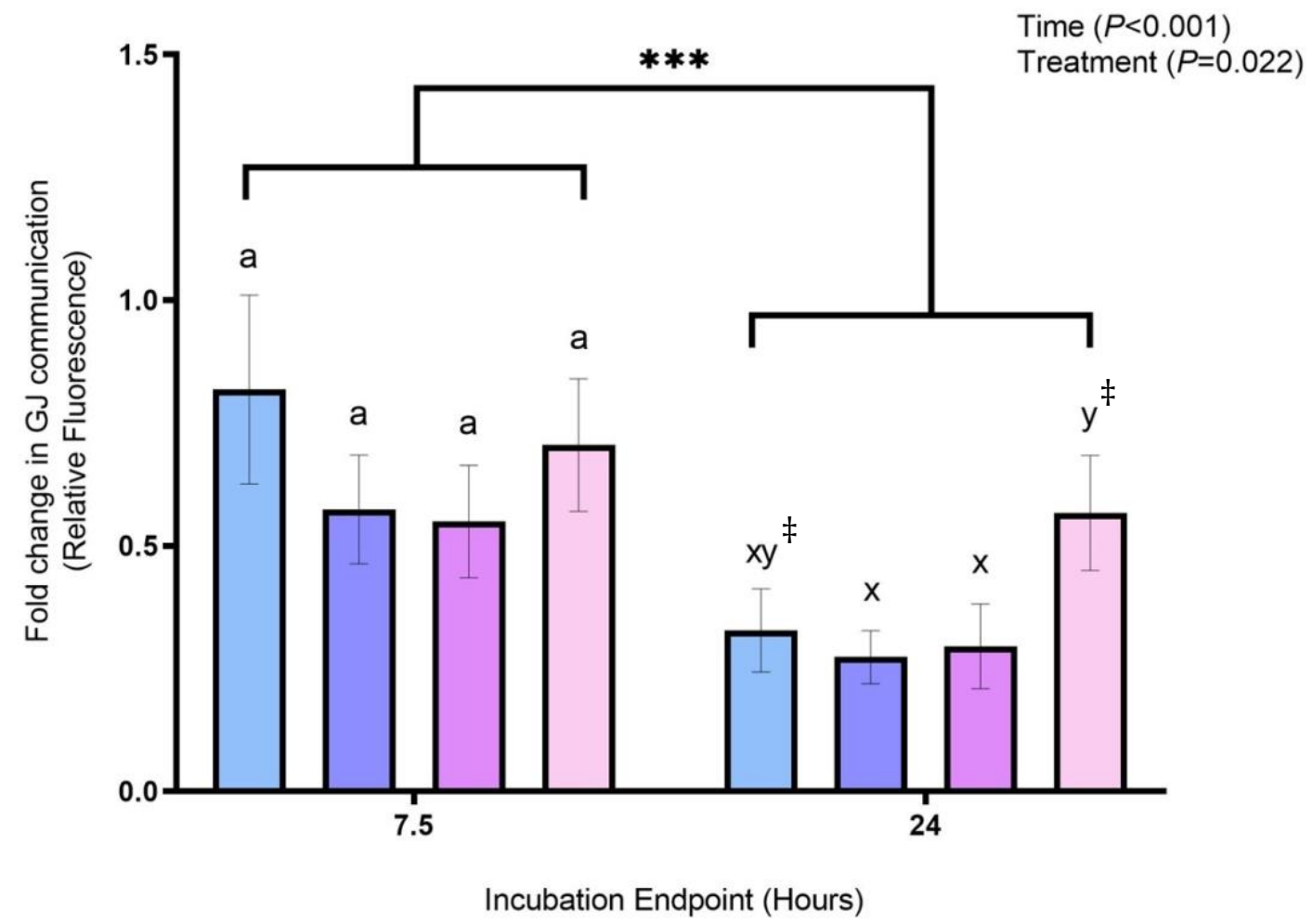

Figure 19: Fold change of gap junction (GJ) communication, represented by intra-oocyte fluorescence levels, in ovine COC incubated in an IVM culture system supplemented with different ratios of GDF9 and BMP15. Control group (no GDF9:BMP15) [ $\square$ ], and

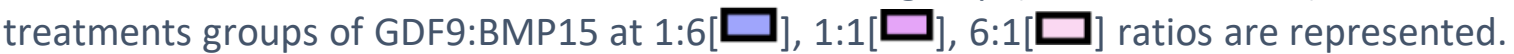
Data were normalised to the 1 hour timepoint and are shown as mean \pm SEM fluorescent levels from four replicate experiments involving six COC per treatment group. A treatment interaction ( $P=0.092$ ) was observed at 24 hours denoted by superscript $(\ddagger)$. Differences in time denoted by $\left({ }^{* * *}\right)$ while significant treatment differences denoted by (GDF9:BMP15 1:6 $x$ and $y, P=0.025 ;$ GDF9:BMP15 1:1 $x$ and $y, P=0.026$ ). 


\subsection{Glutathione Conjugation Assay}

The intra-oocyte florescence levels, as an indirect measure of GSH (reduced glutathione), in ovine COC at 1, 7.5 and 24 hours in a gold-standard IVM system supplemented with different ratios of GDF9:BMP15 or an empty vector control are depicted in Figure 20. Overall, there was a significant time difference in intra-oocyte GSH levels, where GSH increased with time $(P<0.001)$.

Time $(P<0.001)$

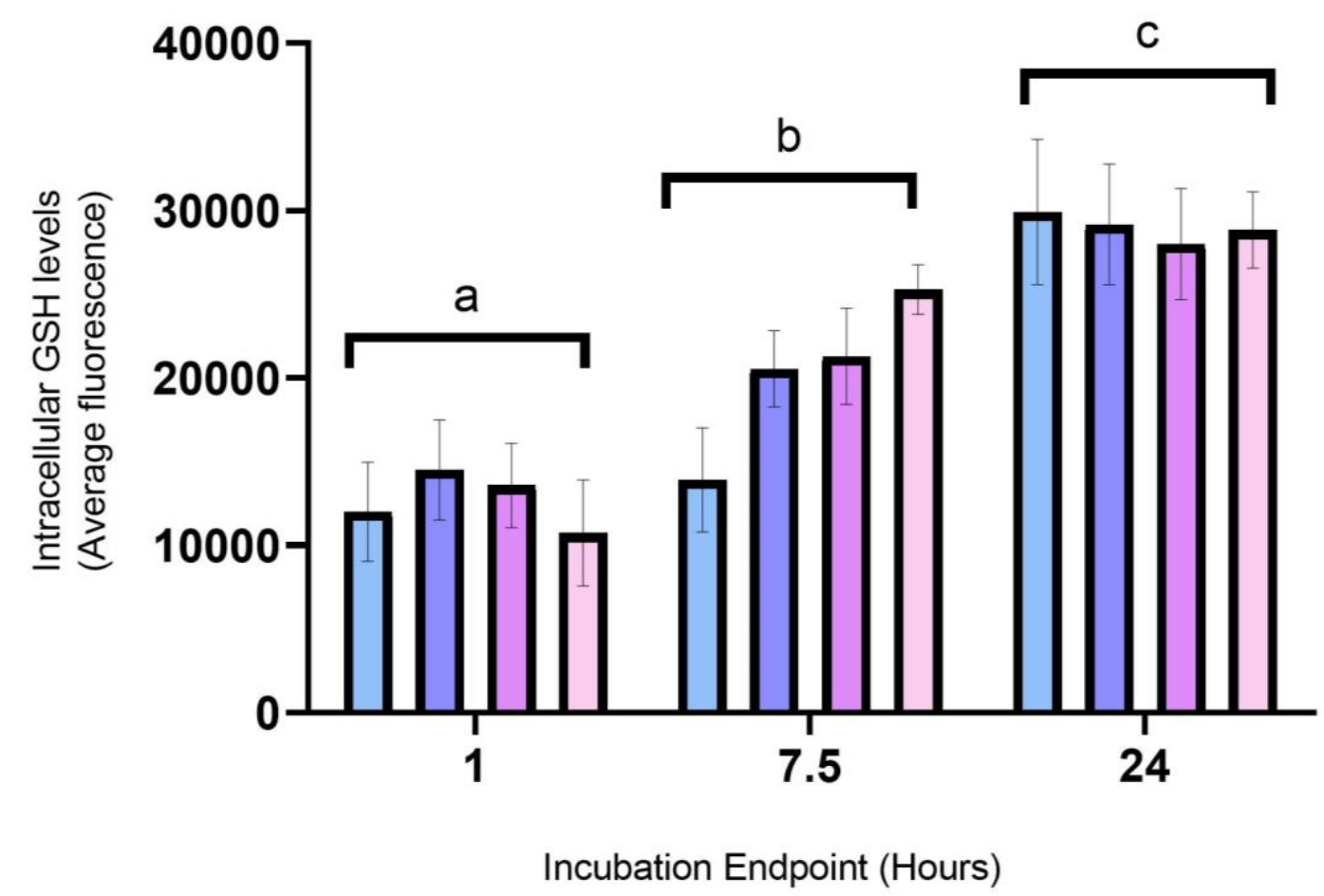

Figure 20: Effect of different ratios of GDF9 and BMP15 in a biphasic IVM culture system on the conjugation levels of lipophilic molecules to reduced glutathione (GSH) in ovine COC. Intra-oocyte fluorescent levels in ovine COC after 1, 7.5 and 24 hours of incubation in a control IVM media system supplemented with differing ratios of oocyte-secreted

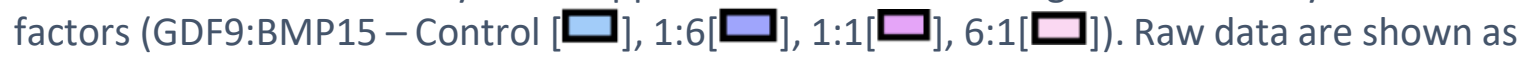
mean \pm SEM fluorescent levels from three replicate experiments involving six COC per treatment group. Two-way ANOVA demonstrates significant differences in time $(P<0.001)$ between relative fluorescence and incubation times denoted by ( $a, b$ and $c)$. 
To account for variability due to seasonality and media preparations between the three biological replicate experiments, data at 7.5 and 24 hours of incubation was also normalised against that at 1 hours incubation (Figure 21). Normalisation of the data in this manner demonstrated a difference $(P=0.001)$ in the fold-change in intra-oocyte GSH levels between the treatment groups at 7.5 , but not 24 , hours in culture. After 7.5 hours in culture, GSH levels were highest in oocytes incubated with a high (6:1) GDF9:BMP15 ratio, compared to 1:6 $(P<0.001)$ and 1:1 $(P=0.019)$ GDF9:BMP15 ratios and the empty vector control $(P<0.001)$. Moreover, GSH concentrations were higher in oocytes cultured in 1:1 ratio of GDF9:BMP15 compared to the 1:6 $(P=0.05)$ and the empty vector $(P=0.01)$.

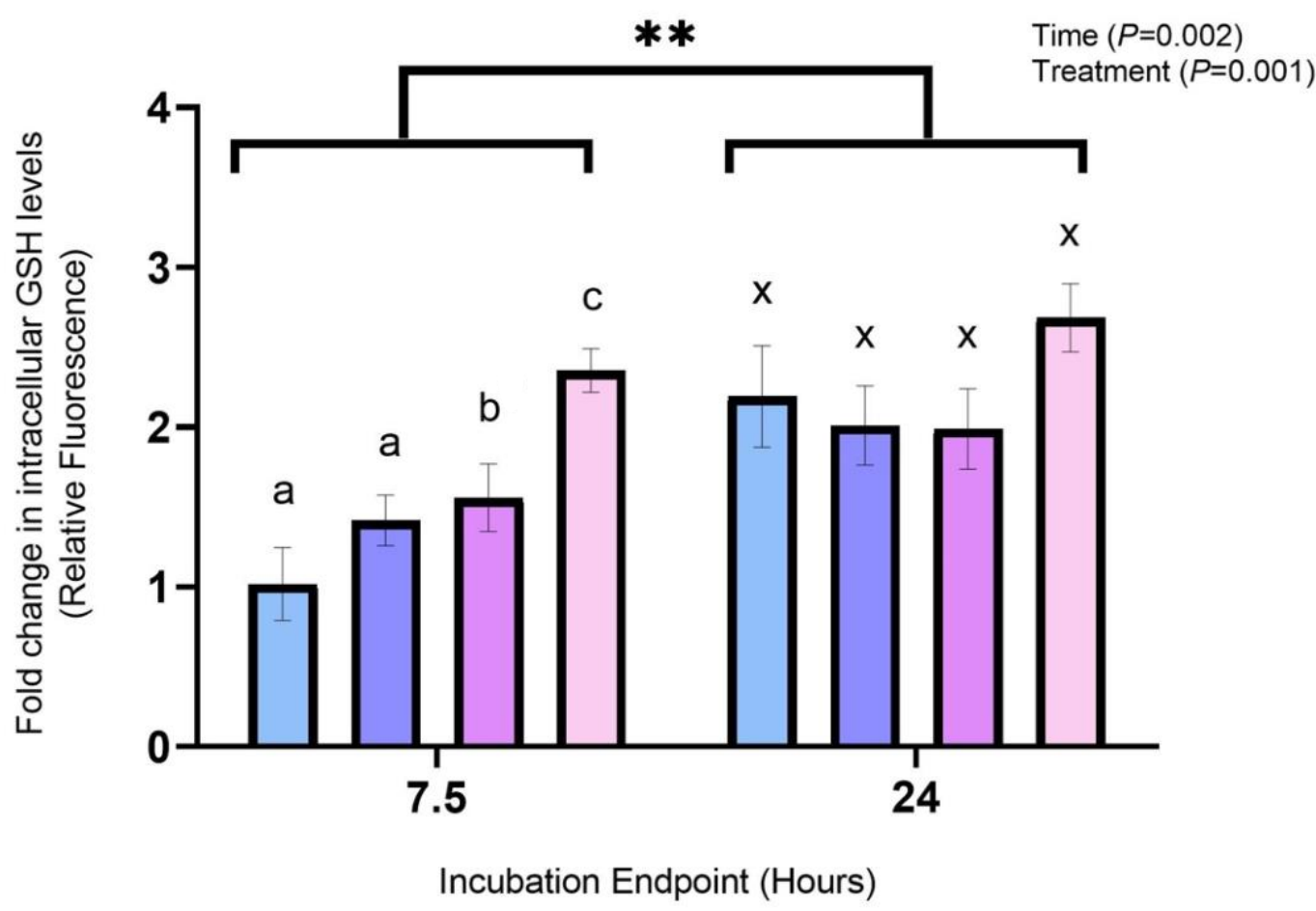

Figure 21: Fold-change of conjugation levels of lipophilic molecules to reduced glutathione (GSH) in ovine COC exposed to different ratios of GDF9 and BMP15 in a biphasic IVM culture system. Data is normalised to 1 hour and shown as mean \pm SEM fluorescent levels from three replicate experiments involving six COC per treatment group Control group (no GDF9:BMP15) [ $\square$ ], and oocyte-secreted factor (OSF) treatment

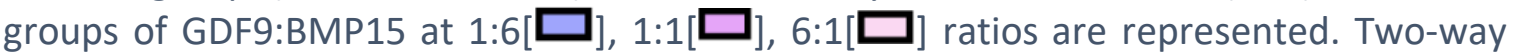
ANOVA demonstrates significant differences in time $(P=0.002)$ and treatment $(P=0.001)$ between relative fluorescence and incubation times. Differences in time denoted by $\left({ }^{* *}\right)$ while significant treatment differences denoted by (GDF9:BMP15 1:1 and 1:6 a and b, $P=0.050$ and $1: 1$ and empty vector, $P=0.01$; GDF9:BMP15 6:1 and empty vector $a$ and $c$, $P<0.001 ; 6: 1$ and $1: 1$ and $c, P<0.001 ; 6: 1$ and $1: 1 \mathrm{~b}$ and $c, P=0.019)$. 


\subsection{Morphological Assessment of Maturation}

\subsubsection{Assessment for Meiotic Resumption}

To assess the effect of treatment on GV and GVBD rates, the observed and expected number of GV and GVBD oocytes were calculated according to treatment after 1, 7.5 and 24 hours in IVM and are represented as contingency tables (Tables 8-10). Chi-squared analyses at 1 hour reported $(\chi=7.815, \mathrm{df}=3)$ with $(P=0.7195)$, at 7.5 hours that $(\chi=1.173$, $\mathrm{df}=3)$ with $(P=0.7596)$ and similarly at 24 hours that $(\chi=1.158, \mathrm{df}=3)$ with $(P=0.7632)$. These results support the null hypothesis and indicate that the addition of OSFs does not significantly alter the rate of GVBD at $1,7.5$ and 24 hours relative to the control.

Table 8: Effect of OSF treatments on GVBD rate at 1 hour in IVM. Values represented observed (expected) values.

\begin{tabular}{cccc}
\hline \multicolumn{5}{c}{ OBSERVED (EXPECTED) } \\
\hline TREATMENT (1 HOUR) & GV & GVBD & TOTAL \\
\hline Control & $27(26.3)$ & $6(6.7)$ & 33 \\
\hline GDF9:BMP15 1:6 & $26(25.5)$ & $6(6.5)$ & 32 \\
\hline GDF9:BMP15 1:1 & $21(23.2)$ & $8(5.8)$ & 29 \\
\hline GDF9:BMP15 6:1 & $25(24.0)$ & $5(6.0)$ & 30 \\
\hline TOTAL & 99 & 25 & 124
\end{tabular}

Table 9: Effect of treatments on GVBD rates at 7.5 hours in IVM. Values represented observed (expected) values.

\begin{tabular}{cccc}
\hline \multicolumn{5}{c}{ OBSERVED (EXPECTED) } \\
\hline TREATMENT (7.5 HOURS) & GV & GVBD & TOTAL \\
\hline Control & $14(14.8)$ & $11(10.2)$ & 25 \\
\hline GDF9:BMP15 1:6 & $15(16.6)$ & $13(11.4)$ & 28 \\
\hline GDF9:BMP15 1:1 & $15(14.8)$ & $10(10.2)$ & 25 \\
\hline GDF9:BMP15 6:1 & $20(17.8)$ & $10(12.2)$ & 30 \\
\hline TOTAL & 64 & 44 & 108
\end{tabular}


Table 10: Effect of treatments on GVBD rates at $\mathbf{2 4}$ hours in IVM. Values represented observed (expected) values.

\begin{tabular}{cccc}
\hline \multicolumn{5}{c}{ OBSERVED (EXPECTED) } \\
\hline TREATMENT (24 HOURS) & GV & GVBD & TOTAL \\
\hline Control & $3(2.2)$ & $21(21.8)$ & 24 \\
\hline GDF9:BMP15 1:6 & $4(3.1)$ & $30(30.9)$ & 34 \\
\hline GDF9:BMP15 1:1 & $2(3.0)$ & $31(30.0)$ & 33 \\
\hline GDF9:BMP15 6:1 & $2(2.7)$ & $27(26.3)$ & 29 \\
\hline TOTAL & 11 & 109 & 120 \\
\hline
\end{tabular}

To assess the effect of incubation time on GVBD rate, the observed and expected number of GV and GVBD oocytes, were calculated and are represented in a contingency table (Table 11). Chi-squared analyses reported that $(\chi=127.9, \mathrm{df}=2)$ with $(P<0.0001)$. From this data, the null hypothesis was rejected. Although there were no differences in the rate of GVBD with the addition to OSFs over the course of IVM, the rate of GVBD increased over time which is concomitant to what is reported in literature.

Table 11: Observing the effect of timepoints on GVBD rates.

\begin{tabular}{cccc}
\hline \multicolumn{5}{c}{ OBSERVED (EXPECTED) } \\
\hline INCUBATION ENDPOINT (HOURS) & $\mathrm{GV}$ & $\mathrm{GVBD}$ & $\mathrm{TOTAL}$ \\
\hline 1 & $99(61.3)$ & $25(62.7)$ & 124 \\
\hline 7.5 & $64(53.4)$ & $44(54.6)$ & 108 \\
\hline 24 & $11(59.3)$ & $109(60.7)$ & 120 \\
\hline TOTAL & 174 & 178 & 352 \\
\hline
\end{tabular}




\subsubsection{Cumulus Cell Expansion}

To assess the effects of treatment on CC grades at each timepoint, the expected and observed number of CC graded 1-3 ( $1=$ Compact, $2=$ Partial and $3=$ Expanded as described in section 2.5.2 and Table 3) were calculated according to treatment and are represented in contingency tables (Tables 12-14). As CC graded as "expanded" were null among all treatment groups at 1 and 7.5 hours, the chi-squared was not possible and thus, this treatment group was removed from the analyses at these timepoints however, was still included at 24 hours. Chi-squared analyses at 1 hour reported $(\chi=2.138, \mathrm{df}=3)$ with $(P=0.5542)$, at 7.5 hours $(\chi=3.280, \mathrm{df}=3)$ with $(P=0.3504)$ and at 24 hours $(\chi=2.893, \mathrm{df}=$ 6) with $(P=0.8221)$. These results support the null hypothesis and indicate that the addition of OSFs does not significantly alter the expansion grading and morphology of CC between treatment groups at $1,7.5$ and 24 hours relative to the control.

Table 12: Observing the effect of treatments on CC expansion at 1 hour in IVM.

\begin{tabular}{cccc}
\hline \multicolumn{5}{c}{ EXPECTED (OBSERVED) } & & \\
\hline TREATMENT (1 HOUR) & $1=$ Compact & 2 = Partial & TOTAL \\
\hline Control & $15(15.7)$ & $18(17.3)$ & 33 \\
\hline GDF9:BMP15 1:6 & $17(15.7)$ & $16(17.3)$ & 33 \\
\hline GDF9:BMP15 1:1 & $10(12.8)$ & $17(14.2)$ & 27 \\
\hline GDF9:BMP15 6:1 & $16(13.8)$ & $13(15.2)$ & 29 \\
\hline TOTAL & 58 & 64 & 122 \\
\hline
\end{tabular}

Table 13: Observing the effect of treatments on CC expansion at 7.5 hours in IVM.

\begin{tabular}{cccc}
\hline \multicolumn{4}{c}{ EXPECTED (OBSERVED) } \\
TREATMENT (7.5 HOURS) & 1 = Compact & 2 = Partial & TOTAL \\
\hline Control & $15(12.9)$ & $7(9.1)$ & 22 \\
\hline GDF9:BMP15 1:6 & $20(17.5)$ & $10(12.5)$ & 30 \\
\hline GDF9:BMP15 1:1 & $12(14.0)$ & $12(10.0)$ & 24 \\
\hline GDF9:BMP15 6:1 & $15(17.5)$ & $15(12.5)$ & 30 \\
\hline TOTAL & 62 & 44 & 106
\end{tabular}


Table 14: Observing the effect of treatments on CC expansion at 24 hours in IVM.

\begin{tabular}{ccccc}
\hline \multicolumn{7}{c}{ OBSERVED (EXPECTED) } & & \\
\hline TREATMENT (24 HOURS) & 1 = Compact & 2 = Partial & 3 = Expanded & TOTAL \\
\hline Control & $6(4.5)$ & $7(6.5)$ & $15\left(17.3^{*}\right)$ & 28 \\
\hline G:B 1:6 & 4 & 8 & $22\left(21.0^{*}\right)$ & 34 \\
\hline G:B 1:1 & 5 & 7 & $16\left(17.2^{*}\right)$ & 28 \\
\hline G:B 6:1 & 4 & 4 & $20\left(17.3^{*}\right)$ & 28 \\
\hline TOTAL & 19 & 26 & 73 & 118 \\
\hline
\end{tabular}

* (rounded values)

To assess the effects of time on CC expansion rates between the timepoints, the observed and expected number of CC graded 1-3 ( 1 = Compact, $2=$ Partial and $3=$ Expanded, as described in Section 2.5.2 and Table 3) were calculated and are represented in a contingency table (Table 15). Chi-squared analyses reported that $(\chi=183.2, \mathrm{df}=4)$ with $(P<0.0001)$. From this data, we can reject the null hypothesis. Though there is no difference in the CC grades between each treatment group over the course of IVM, the rate of CC expansion increases over time which is concomitant to what is reported in literature.

Table 15: Observing the effect of timepoint on CC expansion.

\begin{tabular}{ccccc}
\hline \multicolumn{7}{c}{ OBSERVED (EXPECTED) } \\
\hline $\begin{array}{c}\text { INCUBATION ENDPOINT } \\
\text { (HOURS) }\end{array}$ & 1 Compact & 2 = Partial & 3 = Expanded & TOTAL \\
\hline 1 & $58(49)$ & $64(47.2)$ & $0\left(25.7^{*}\right)$ & 122 \\
\hline 7.5 & $62(42.6)$ & $44(41.1)$ & $0\left(22.4^{*}\right)$ & 106 \\
\hline 24 & $19(47.4)$ & $26(45.7)$ & $73\left(24.9^{*}\right)$ & 118 \\
\hline TOTAL & 139 & 134 & 73 & 346 \\
\hline
\end{tabular}

\footnotetext{
* (rounded values)
} 


\subsection{Glycolytic Metabolites in Media}

\subsubsection{Optimisation of Pyruvate Consumption Bioassay}

A simple, high-throughput, ultrafluorometric plate-based bioassay was established to detect changes in pyruvate in IVM media to reflect consumption by the ovine COC. A standard curve of sodium pyruvate was used to standardise the IVM media against.

\subsubsection{Pyruvate Standard in Phenol Red versus Phenol Red-Free Media}

Standard curves of sodium pyruvate showed a strong positive correlation between fluorescence values and pyruvate concentrations, due to the oxidation of NADH with both phenol red $\left(y=9239 x+3895.5, R^{2}=0.9841\right)$ and phenol red-free conditions as depicted in Figure $22\left(y=8124 x+4853.4, R^{2}=0.9945\right)$. Further statistical analyses through linear regression demonstrated that there was no significant difference between the two conditions ( $F=1.32425, \mathrm{df}=1,5)$.

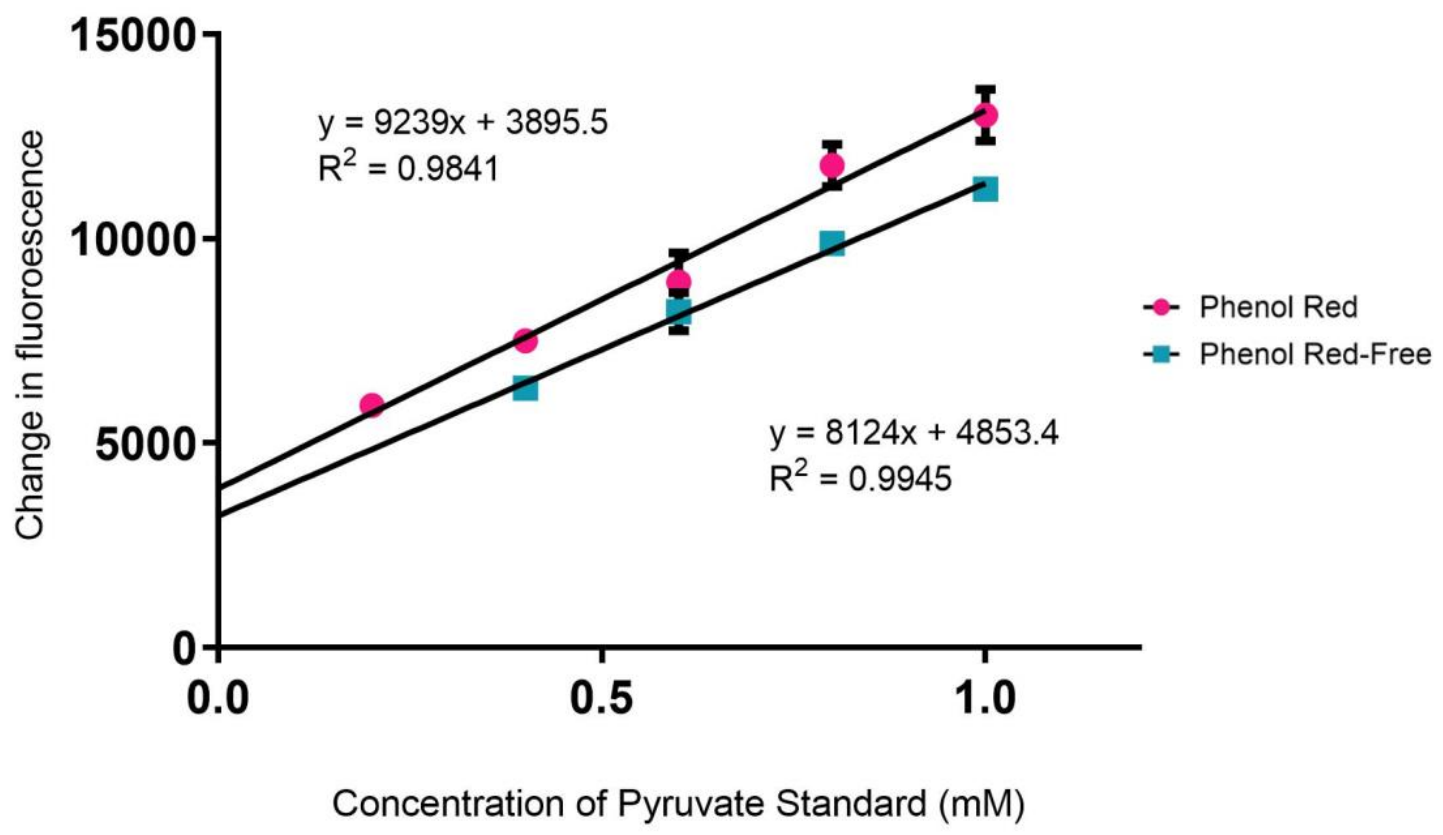

Figure 22: Sodium pyruvate standards tested in phenol-red and phenol-red free conditions to establish a standard curve. The ability for the fluorescent detection of sodium pyruvate in phenol red $(\bullet)$ and phenol red-free ( $\square$ ) media conditions were compared through linear regression. Data are presented as mean \pm SEM from three replicate experiments 


\subsubsection{Fresh versus Freeze-Thawed Assay Mixture}

Standard curves of sodium pyruvate showed a strong positive correlation between fluorescence values and pyruvate concentrations, due to the oxidation of NADH with both fresh $\left(y=5729 x+4835.1, R^{2}=0.9899\right)$ and freeze-thawed assay mixture $(y=1489 x+$ 368.7, $\left.R^{2}=0.9926\right)$, as depicted in Figure 23. However further statistical analyses through linear regression demonstrated that the assay depicting the fresh preparation exhibited a greater level of sensitivity to changes in fluorescence than the freeze-thawed assay stock ( $F=153.889, \mathrm{df}=1,6 ; P<0.0001)$.

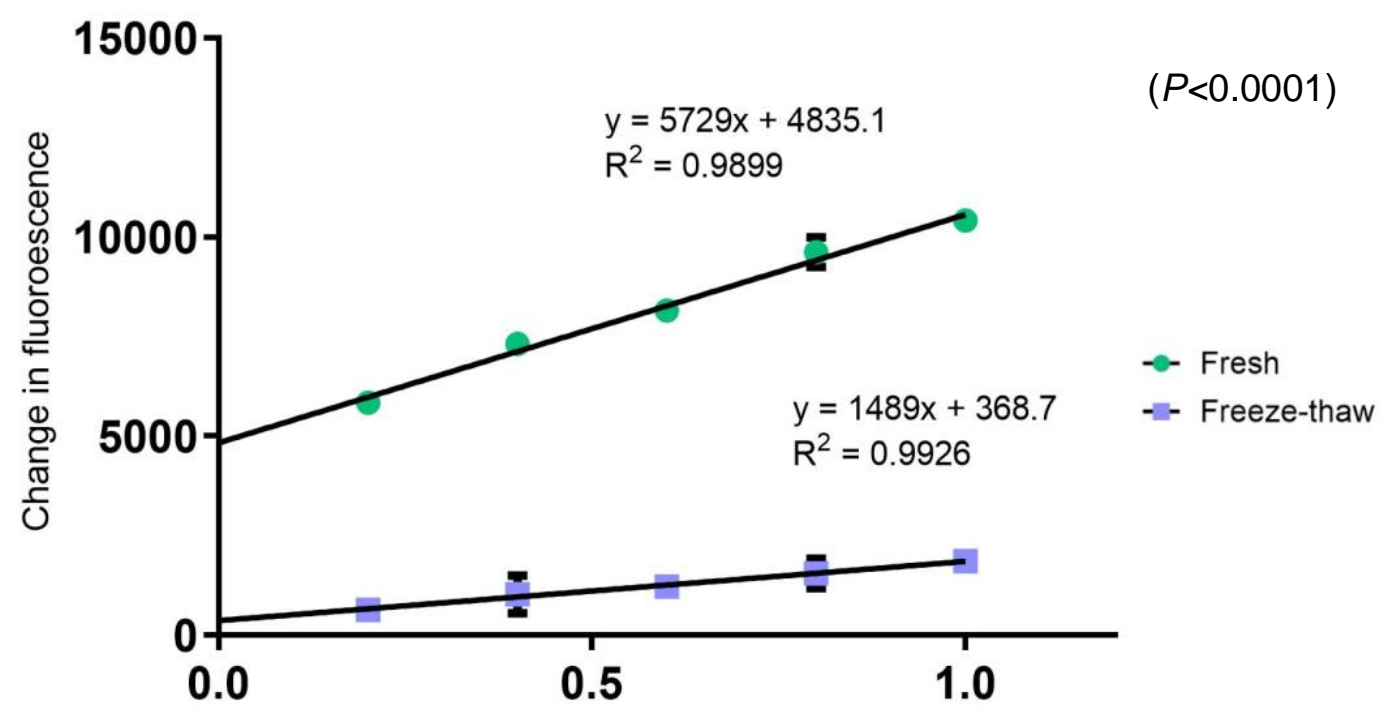

Concentration of Pyruvate Standard (mM)

Figure 23: Sodium pyruvate standards tested in fresh and freeze-thawed enzyme assay mix to establish a standard curve. The ability for the fluorescent detection of sodium pyruvate in fresh ( $\bullet$ ) and freeze-thawed ( $\square$ ) assay mixtures were compared through linear regression which was able to establish a significant difference in fluorescence detection between the two conditions $(P<0.0001)$. Data are presented as mean \pm SEM from three replicate experiments. 


\subsubsection{Pyruvate Consumption by Ovine COC}

Pyruvate consumption levels by ovine COC in IVM media supplemented with different ratios of GDF9:BMP15 or empty vector control at 1, 7.5 and 24 hours are depicted in Figure 24. Overall, there was a treatment $(P<0.001)$ difference in pyruvate consumption, particularly during the 7.5 and 24 hour incubation timepoints $(P=0.001)$. Post-hoc tests demonstrated that a high GDF9:BMP15 ratio resulted in higher consumption rates after 7.5 hours in IVM, relative to the empty vector control $(P<0.001)$. At 24 hours, both high GDF9:BMP15 $(P<0.001)$ and low GDF9:BMP15 $(P=0.004)$ ratios demonstrated higher consumption rates relative to the empty vector control. A near significant interaction was observed at 24 hours where 1:1 ratios showed increased consumption rates relative to the control $(P=0.055)$. Interestingly at 1 hour, an interaction was also observed where the high GDF9:BMP15 treatment showed relatively higher $(P=0.064)$ consumption levels relative to the 1:1 ratio. 


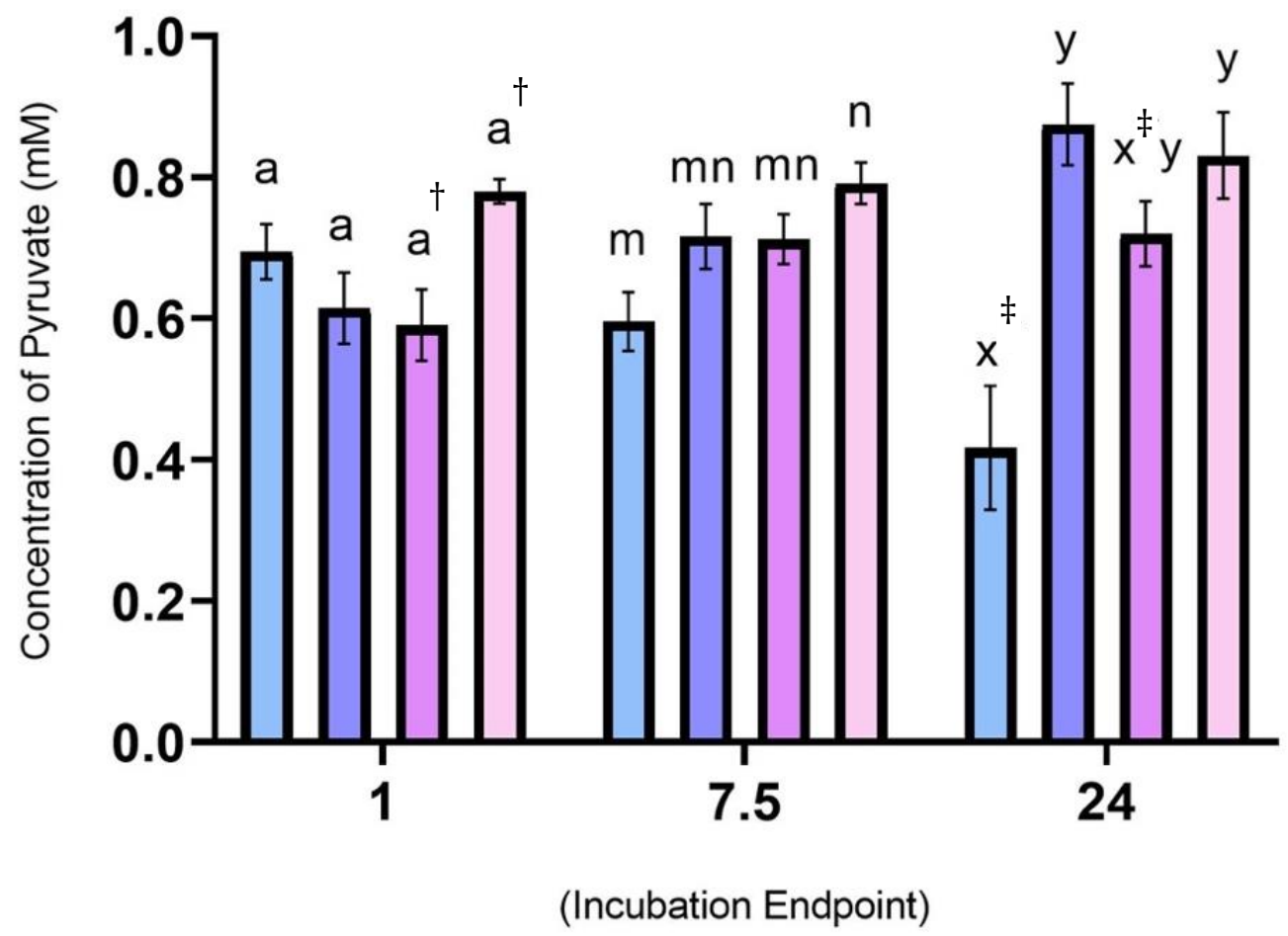

Figure 24: Effect of different ratios of GDF9 and BMP15 in a biphasic IVM culture system on the rate of pyruvate consumption by ovine COC. Changes in pyruvate concentrations in IVM media relative to rate of pyruvate consumption by ovine COC after 1, 7.5 and 24 hours of incubation in a biphasic IVM system supplemented with differing ratios of

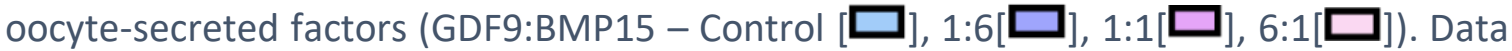
are shown as mean \pm SEM concentrations from seven replicate experiments involving six media droplets per treatment group. Two-way ANOVA demonstrates significant differences in time*treatment $(P=0.001)$ and treatment $(P<0.001)$. Significant treatment differences denoted by (GDF9:BMP15 6:1 and empty vector $m$ and $n, P<0.002$; and 1:6 and empty vector $x$ and $y, P=0.004$; GDF9:BMP15 6:1 and empty vector $x$ and $y, P<0.001$ ). Interactions denoted by $(\dagger, \neq, P=0.064 ;, P=0.055)$. 


\subsection{Gene Expression Analyses}

\subsubsection{Changes in Key Oocyte Genes}

The mRNA levels of GDF9, BMP15, HIF1A, SOD1 and PRDX6 in single ovine oocytes incubated for 1, 7.5 or 24 hours in an IVM system supplemented with differing ratios of GDF9:BMP15 are shown in Figure 25. There were no significant differences in levels of GDF9, BMP15 and SOD1 due to time or treatment. In contrast, mean HIF1A levels were upregulated $(P=0.004)$ after 7.5 hours in culture, relative to those oocytes cultured for 1 and 24 hours. Mean PRDX6 levels were higher at 7.5 and 24 hours, compared to 1 hour, in culture $(P<0.001)$. 

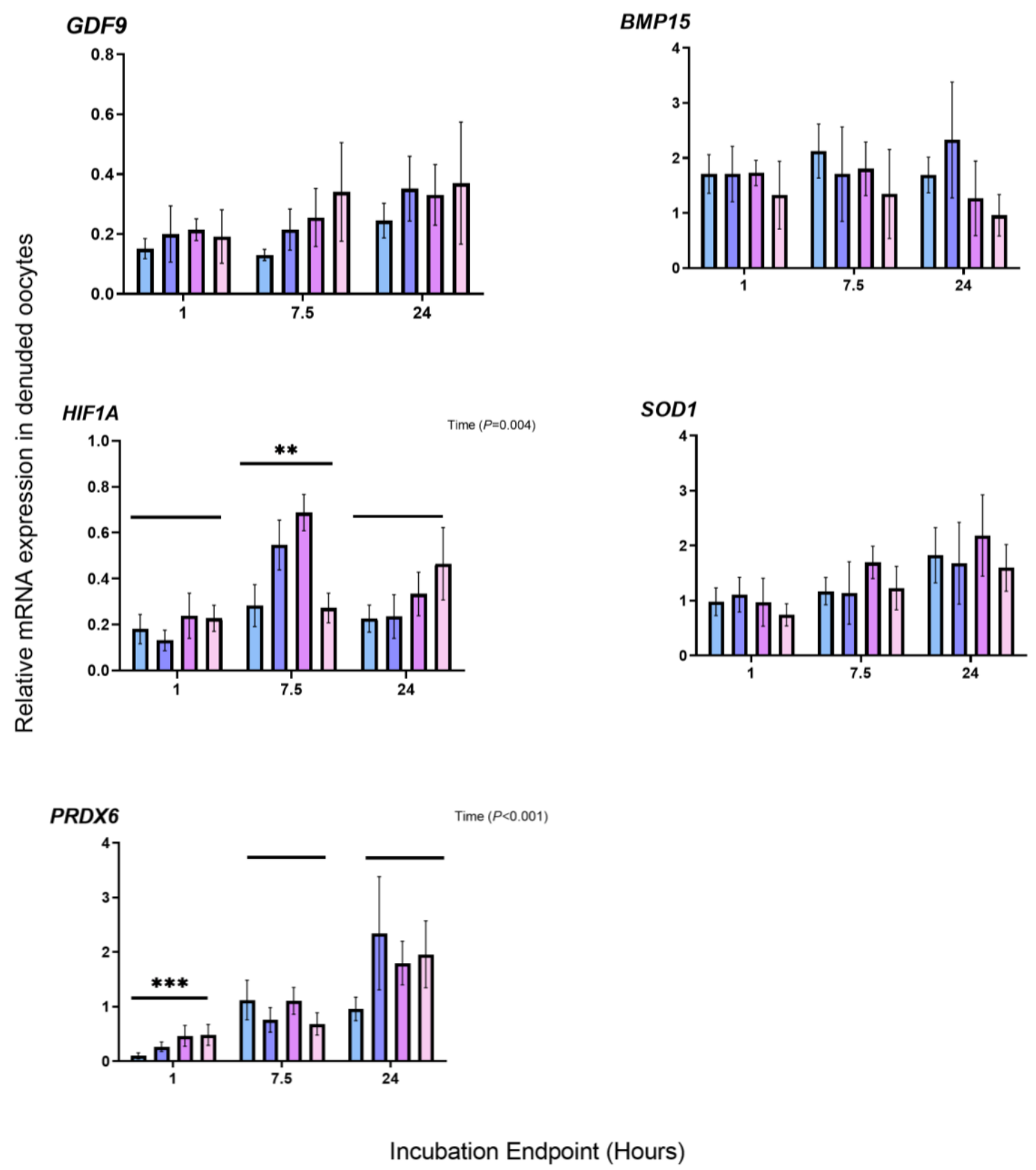

Figure 25: Effect of GDF9 and BMP15 ratios on relative mRNA expression levels in ovine oocytes following incubation in a biphasic IVM culture system. Changes in relative mRNA expression for GDF9, BMP15, HIF1A, SOD1 and PRDX6 in ovine oocytes incubated at 1, 7.5 and 24 hours in a biphasic IVM system supplemented with differing ratios of oocyte-

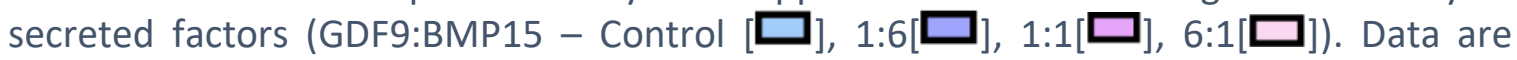
presented as mean \pm SEM from seven replicate experiments involving two COC per treatment group. Significant time differences resulting from a two-way ANOVA are denoted by $(* *, * * *)$. 


\subsubsection{Changes in Key Cumulus Cell Genes}

The mRNA levels of BAX, BCL2, EGFR, FSHR, PR, CX43, GSTA1 and TNFAIP6 in CC of ovine COC incubated for $1,7.5$ or 24 hours in an IVM system supplemented with differing ratios of GDF9:BMP15 are shown in Figure 26. Significant time effects were observed in all genes except for EGFR. Mean BAX and BCL2 levels were higher $(P<0.001)$ at 24 hours, compared to that after 1 hour in culture. The expression of $B A X$ mRNA was also shown to be influenced by the ratio of GDF9:BMP15, where the addition of OSFs, regardless of ratio, resulted in an upregulation of mean $B A X$ mRNA levels at 24 hours $(1: 6 P<0.01 ; 1: 1$ $P=0.017 ; 6: 1 P<0.001)$. Interestingly, a treatment interaction was also observed at 1 hour in culture, where a higher GDF9:BMP15 ratio showed relatively upregulated expression of $B A X$ mRNA, relative to the control. However, $B A X: B C L 2$ ratio must be considered for a more accurate representation of apoptotic state (further discussed in Section 3.5.3, Figure 27). Mean $C X 43$ levels were elevated after 7.5 and 24 hours, compared to 1 hour in culture $(P<0.001)$. An overall treatment effect was also observed where oocytes incubated in the GDF9:BMP15 ratio of 6:1 exhibited the higher $C X 43$ levels relative to the empty vector control after 24 hours in culture $(P<0.01)$. A near significant treatment interaction was also observed at 1 hour in incubation where the higher BMP15 ratio showed elevated expression of $C X 43$ relative to the empty vector control $(P=0.055)$. Interestingly, mean GSTA1 were negatively correlated with time in culture with lower levels measured in CC after 7.5 and 24 hours $(P=0.027)$. Mean FSHR mRNA, regardless of treatment, demonstrated an overall upregulation in expression at 7.5 hours relative to mRNA expression levels at 1 hour in culture. Mean TNFAIP6 mRNA showed a significant upregulation in expression levels at 24 hours in comparison to 1 hour in culture, regardless of treatment $(P<0.001)$. Similarly, mean $P R$ mRNA demonstrated increased levels of expression at 24 hours relative to both 1 and 7.5 hours. Mean EGFR mRNA demonstrated an overall treatment effect $(P<0.05)$. Upon further interrogation via Bonferroni and Tukey HSD post-hoc tests, it was demonstrated that both high and low GDF9:BMP15 ratios resulted in an upregulated expression of mean EGFR levels at 24 hours. Interestingly, a treatment interaction can be observed at 1 hour where the low GDF9:BMP15 ratio also showed an elevated expression of EGFR levels, relative to the empty vector control. 

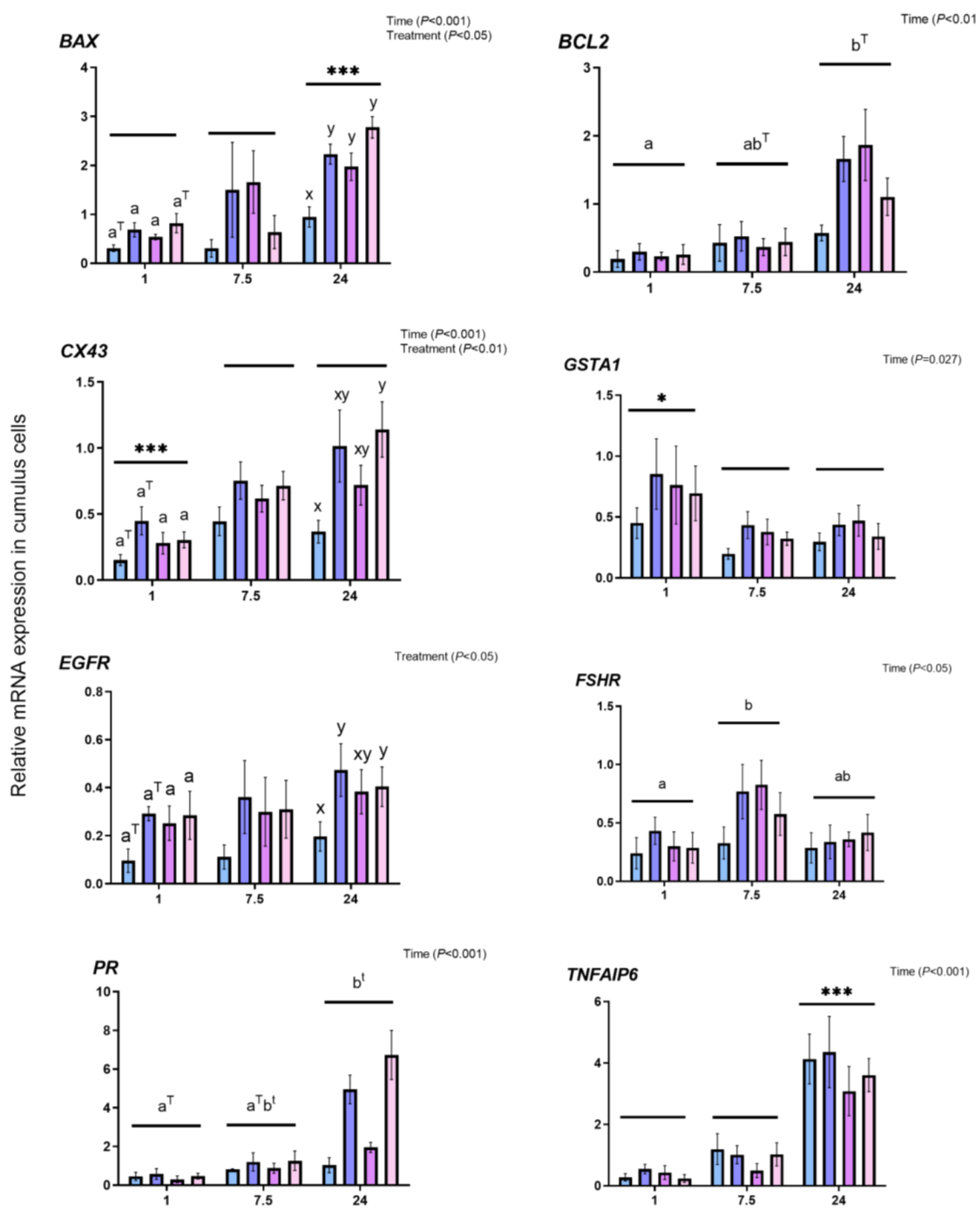

Incubation Endpoint (Hours)

Figure 26: Effect of GDF9 and BMP15 ratios on relative mRNA expression levels in ovine cumulus cells $(C C)$ following incubation in a biphasic IVM culture system. Changes in relative mRNA expression for BAX, BCL2, CX43, GSTA1, EGFR, FSHR, PR and TNFAIP6 in ovine CC incubated at 1, 7.5 and 24 hours in a biphasic IVM system supplemented with

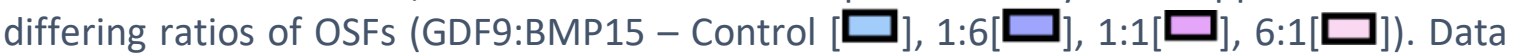
are presented as mean \pm SEM from seven replicate experiments involving two COC per treatment group. Significant time and treatment differences resulting from a two-way ANOVA are denoted by $(*, * * *, a, b, x, y)$. Treatment interactions are denoted by superscript ( $T$ and $t)$. 


\subsubsection{Ratios in Functionally Related Genes}

The change in $B A X: B C L 2$ ratio in CC of ovine COC incubated at 1, 7.5 and 24 hours in a biphasic IVM system supplemented with differing ratios of GDF9:BMP15 are depicted in Figure 27. Overall, time $(P<0.001)$, treatment $(P<0.001)$ and timeXtreatment differences associated with specific timepoints $(P=0.001)$ were observed. At 7.5 hours in culture, the empty vector control demonstrated upregulated ratios of $B A X: B C L 2$ relative to all the GDF9:BMP15 ratios $(1: 6, P<0.05 ; 1: 1, P=0.001 ; 6: 1 P=0.017)$. At 24 hours, the high GDF9:BMP15 ratio demonstrated significantly downregulated ratios of $B A X: B C L 2$ relative to the empty vector control $(P<0.05)$. Treatment interactions were also observed at this timepoint where the high BMP15 ratio also showed downregulated expression of $B A X: B C L 2(P=0.068)$.

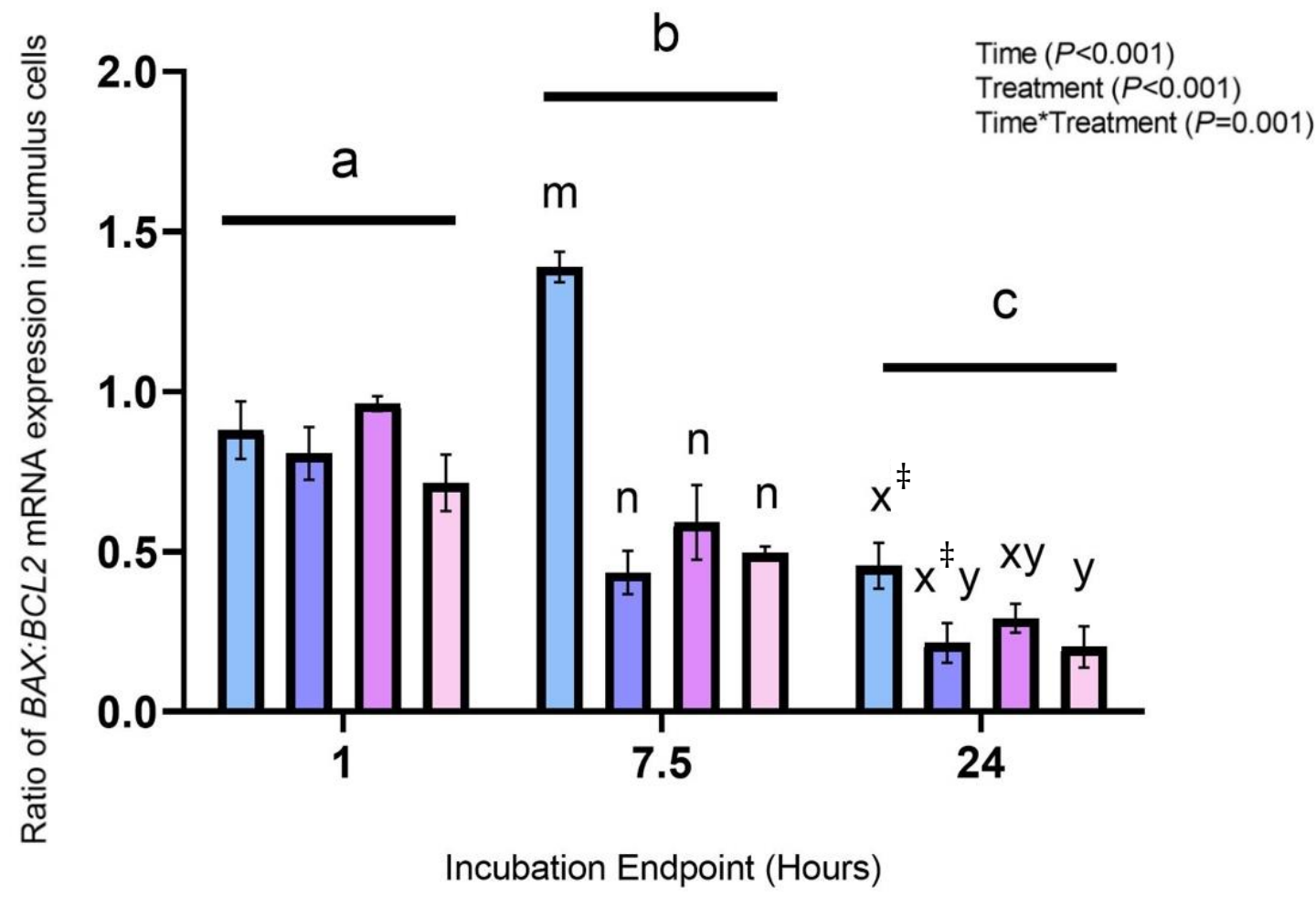

Figure 27: Effect of GDF9 and BMP15 ratios on relative mRNA expression levels on $B A X: B C L 2$ in ovine cumulus cells (CC) following incubation in a biphasic IVM culture system. Changes in relative mRNA expression for BAX:BCL2 in ovine CC incubated at 1, 7.5 and 24 hours in a biphasic IVM system supplemented with differing ratios of oocyte-

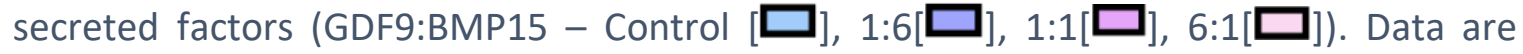
presented as mean \pm SEM from seven replicate experiments involving two COC per treatment group. Significant time and treatment differences resulting from a two-way ANOVA are denoted by $(a, b, c, m, n, x$ and $y)$. Treatment interactions are denoted by superscript (†). 


\subsection{Granulosa Cell Proliferation}

The proliferation rate of GC after incubation with different ratios of GDF9:BMP15 are shown in Figure 28. Overall, the addition of GDF9 and BMP15, regardless of ratio resulted in increased GC proliferation, relative to the empty vector control $(P<0.001)$.

Treatment $(P<0.001)$

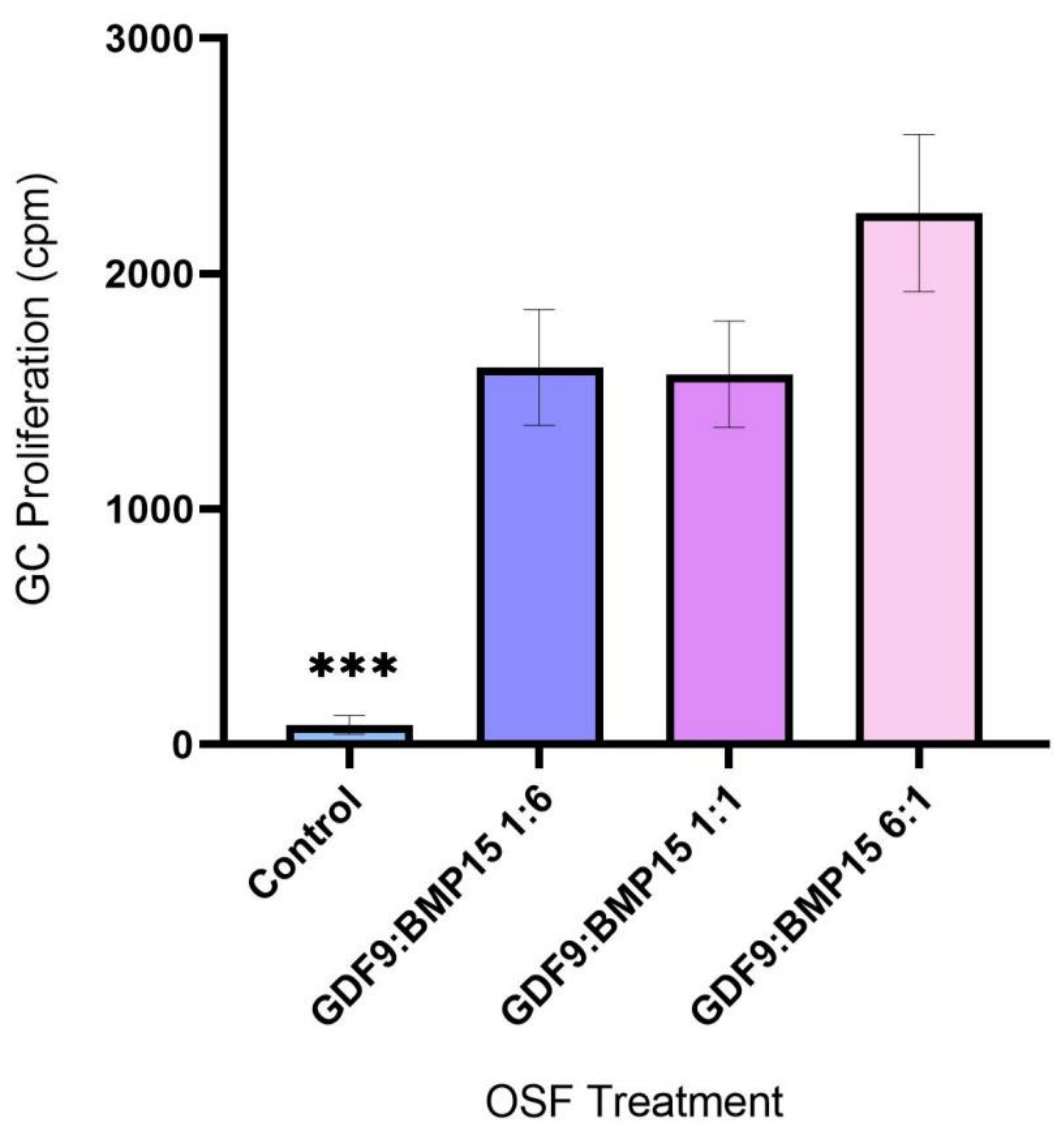

Figure 28: Effect of GDF9 and BMP15 ratios on the proliferation of granulosa cells (GC). Differences in the proliferation rate (cpm; counts per minute) of granulosa cells incubated in growth supplemented with differing ratios of oocyte-secreted factors (GDF9:BMP15 -

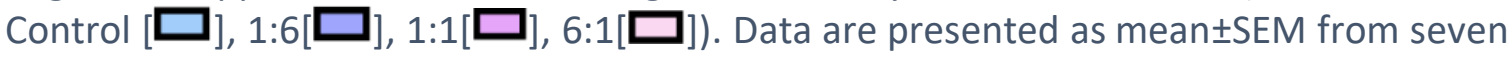
replicate experiments. Significant treatment differences resulting from a two-way ANOVA are denoted by $(* * *)$. 
To account for variability due to seasonality and media preparations between the four biological replicate experiments, all treatments were normalised against data of the control for each replicate experiment (Figure 29). Normalisation of data in this manner did reveal treatment differences in GC proliferation rate, although the highest GDF9:BMP15 ratio appeared to elicit the greatest proliferation rate.

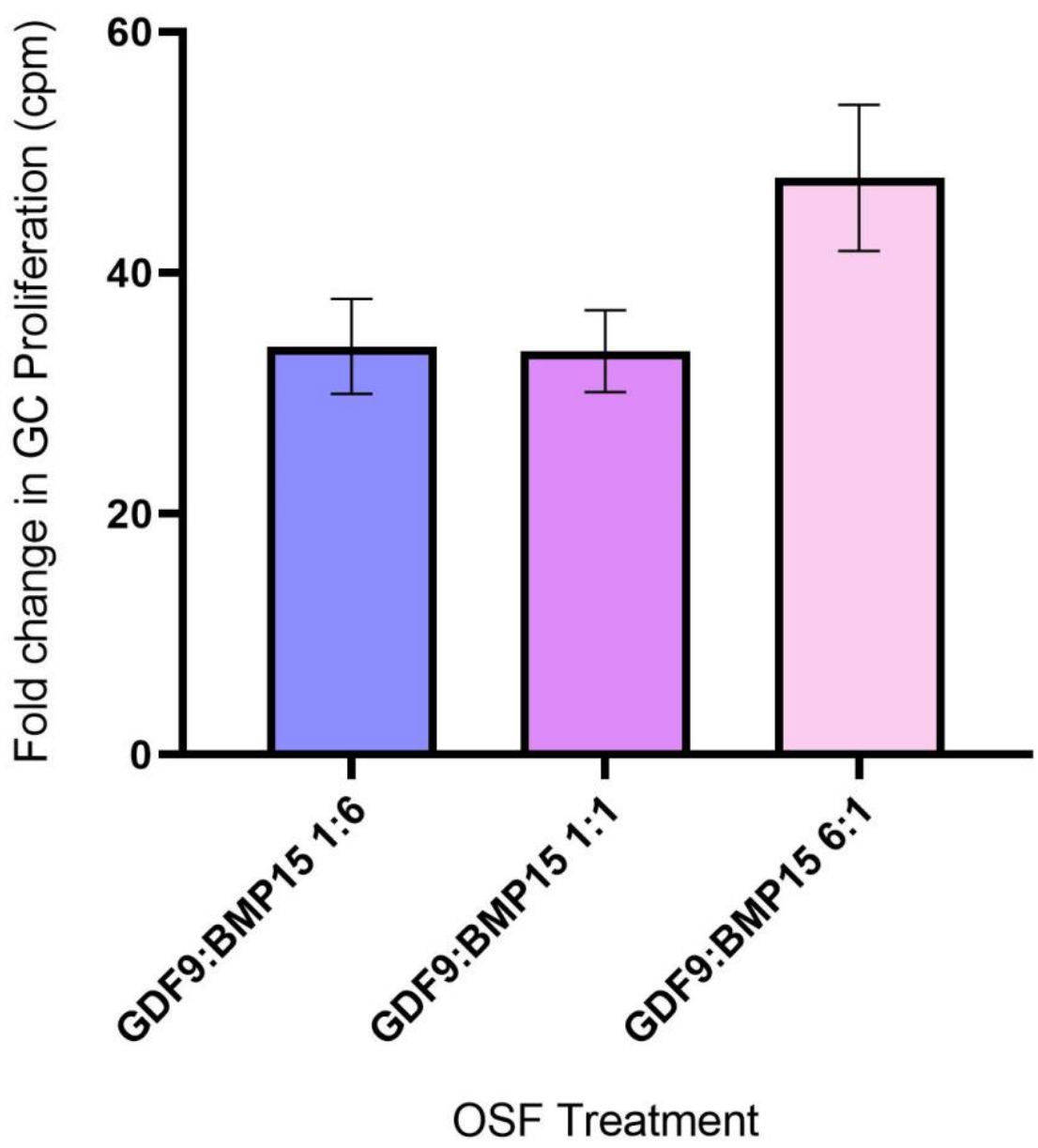

Figure 29: Effect of GDF9 and BMP15 ratios on the fold-change in proliferation of granulosa cells (GC). Fold change in the proliferation rate (cpm; counts per minute) of granulosa cells incubated in growth supplemented with differing ratios of oocyte-secreted

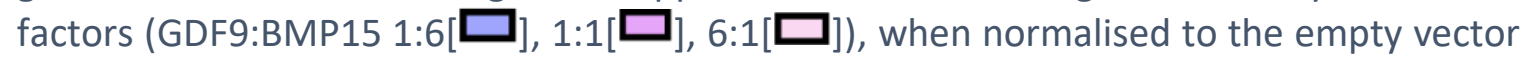
control. Data are presented as mean \pm SEM from seven replicate experiments. 


\section{CHAPTER 4: GENERAL DISCUSSION}

One of the most significant factors impeding the improvement of ART is insufficient knowledge surrounding the attainment of oocyte developmental competency. Successful completion of nuclear and cytoplasmic maturation events in vivo requires a constant synchrony and cross-talk of key endocrine and paracrine factors within the $\mathrm{COC}$ and the entire ovarian follicle. The COC removed from antral follicles are capable of completing nuclear maturation (resumption of meiosis) using commercially viable IVM methods, however completion of cytoplasmic maturation is hindered as this cross-talk is not maintained due to deficiencies in the media. Due to the lack of crucial factors such as the OSFs, in particular GDF9 and BMP15, to maintain molecular events within in vitro systems, the COC undergoes spontaneous breakdown of the GV, and premature resumption of meiosis. As a result of the $\mathrm{COC}$ having insufficient time to store crucial proteins, mRNA and energy substrates, early embryonic events are severely compromised.

This study investigated the effects of using a biphasic IVM media, that has been supplemented with GDF9 and BMP15 at three divergent ratios, on key events involved in oocyte maturation. These GDF9:BMP15 ratios, i.e. 1:6, 1:1 and 6:1, are equivalent to those expressed in mammals that exhibit low, low-moderate and high litter sizes, respectively. Overall, these studies provide novel results on the effects of differing ratios of GDF9:BMP15 on key events involved in nuclear and cytoplasmic maturation, such as GJ activity, intra-oocyte GSH levels, changes in COC morphology, the consumption of glycolytic metabolites by the COC, GC proliferation and expression levels of gene biomarkers of maturation and developmental competency in vitro.

The main findings of this study were that a higher GDF9:BMP15 ratio $(6: 1)$, equivalent to that in a poly-ovulatory species, demonstrated a higher level of reagent transfer to the ovine oocyte through GJs after 24 hours of incubation. Similarly, it was also observed that at the higher ratio, conjugation of lipophilic reagents to GSH was higher at 7.5 hours of incubation. The addition of OSFs, regardless of ratio dose, also facilitated an increased consumption of pyruvate by the $\mathrm{COC}$ relative to the OSF control, although the high GDF9:BMP15 treatment showed greater rates of consumption throughout the culture period. 
The expression of candidate genes in the oocyte (PRDX6) and CC (PR, TNFAIP6 and CX43) increased as maturation progressed. Importantly, the exposure of $\mathrm{COC}$ to high GDF9:BMP15 ratios resulted in higher expression of the GJ encoding gene $C X 43$ at 24 hours relative to all other treatments. Both HIF1A and FSHR in the oocyte and CC, respectively, showed increased expression in all OSF treatments at 7.5 hours, but decreased to basal levels by 24 hours. Regardless of OSF ratios, the addition of GDF9 and BMP15 in IVM media caused an increase in the expression levels of EGFR in the CC, relative to the control treatment. Calculation of the $B A X: B C L 2$ ratio as a measure of the $C C$ susceptibility to apoptosis showed a decrease with COC maturation suggesting that the addition of OSFs, regardless of ratio, protected against cell death. In contrast but as expected, the majority of the candidate genes in the oocyte, namely GDF9, BMP15 and SOX1, were variable and not influenced by treatment. It was also found that the addition of OSFs to GC in culture, regardless of ratio, heavily influenced the proliferation of GC, relative to the no-OSF control treatment. 


\subsection{Effect of GDF9 \& BMP15 on Cytoplasmic Maturation in IVM}

The failure to maintain and complete cytoplasmic maturation is arguably the most significant limitation impeding the success of IVM. Throughout cytoplasmic maturation, mRNA, proteins, organelles and metabolites are trafficked into the oocyte through GJ networks and transzonal projections (Uhde et al., 2018), and can be exploited to meet the energy demands of the oocyte, and prepare for early embryonic events of the transcriptionally-quiescent embryo prior to the activation of its own genome. Changes in these molecules can serve as beneficial biomarkers to determine oocyte quality and the progression of cytoplasmic maturation throughout different points of the incubation period.

\subsubsection{The Effect of GDF9 \& BMP15 on Metabolite Consumption}

In this study, we found that the addition of GDF9 and BMP15, regardless of dose, promoted the consumption of pyruvate by the $\mathrm{COC}$ relative to the control, which appeared to decrease after 7.5 hours. Overall, the rate of pyruvate consumption by COCs did not change over the course of the IVM period. However, a timeXtreatment interaction was observed which showed a treatment effect at the later incubation times. At both 7.5 and 24 hours, the effects of GDF9 on the rate of pyruvate consumption by COCs was dosedependent. At 1 hour, an interaction could be observed in which COCs incubated in the high GDF9:BMP15 ratio were also consuming higher rates of pyruvate than the low GDF9:BMP15 ratio treatments and control. Interestingly, both high and low GDF9:BMP15 treatments had greater rates of pyruvate consumption relative to the control and the 1:1 GDF9:BMP15 treatment, though an interaction in which the 1:1 ratio was also greater than the control, could also be observed at this timepoint. Surprisingly, the expected 2fold increase in the rate of consumption by mature oocytes at 24 hours of incubation was not observed, as has been previously reported (Sutton et al., 2003; Harris et al., 2009). Though this effect was dependent on oxygen availability, it could also be a species-specific difference which would be interesting to investigate further.

Interestingly, these findings are concomitant to previous reports which have insinuated that GDF9 in particular, facilitates energy substrate uptake and transport to the oocyte (Sugiura \& Eppig, 2005). As we know, the oocyte itself does not have the capacity to 
metabolise glucose and relies on the $\mathrm{CC}$ to convert glucose into pyruvate and other intermediate substrates which can be transported to the oocyte through GJ networks and transzonal projections to be further utilised for energy in the form of ATP. Pyruvate is known to be crucial for early embryo cleavage events and blastocyst development (Brinster, 1969). Our results suggest that COCs matured in a high GDF9:BMP15 supplemented medium have a greater capacity to support early embryonic events due to higher pyruvate consumption. Greater pyruvate storage would allow for not only the completion of cleavage events but also increased chances of blastocyst development. This supports experiments conducted by Clark (2018) which found that a high GDF9:BMP15 ratio lead to increased blastocyst rates following IVM in sheep.

While these results truly highlight the importance of OSFs on metabolite consumption in preparation for embryonic development, accurate conclusions cannot be made based on the results of pyruvate consumption alone. In terms of glycolytic carbohydrates and substrates, the consumption of glucose and production of lactate must also be considered in order to determine the full metabolic state of the COC. Non-invasive protocols similar to the methodology for pyruvate have been described to measure changes of glucose and lactate in embryo culture media by Guerif et al. (2013) and McKeegan (2015). These assays could be further optimised for IVM media in order to attain results for these other metabolites

\subsection{2. $\quad$ Effect of GDF9 \& BMP15 on Oxidative Stress}

As a consequence of being metabolically active, there is an increased production of ROS within the ooplasm. In most instances, high levels of ROS have severe effects on the cell due to the promotion of oxidative stress, cell death, maternal aging and ultimately compromise developmental competency. One of the ways in which detrimental effects of ROS is assessed is through the expression levels of genes involved in regulating intracellular stress responses.

HIF1A is an oocyte-specific indicator of the hypoxic state within the ooplasm. In this study, HIF1A mRNA levels were elevated in oocytes at 7.5 hours, relative to 1 and 24 hours, regardless of treatment. This gene is upregulated under hypoxic microenvironments and interestingly regulates the expression and downstream events of vascular endothelial 
growth factor (VEGF ; (Douglas et al., 2005; Takahashi et al., 2016). VEGF regulates cyclic angiogenesis which is necessary for both follicular maturation and the development of the corpus luteum. Previous studies have also reported that the blockage of HIF genes and transcription factors supresses ovulation and reduces antrum formation (Kim et al., 2009). Therefore, the increase of HIF1A observed at 7.5 hours is likely necessary for follicular maturation, particularly at the timepoint were the $\mathrm{COC}$ is undergoing rigorous changes due to on-going maturation. It is also important to highlight, as stated above, that oxygen has been reported to be necessary for the consumption of metabolites such as pyruvate and glucose (Sutton et al., 2003; Harris et al., 2009). By 24 hours, HIF1A is significantly downregulated but still perhaps expressed at a level that, in an in vivo situation, would initiate ovulatory events together with genes such as VEGF. Low levels of HIF1A by the end of IVM has been reported to be a positive biomarker for oocyte developmental competency and blastocyst development (Douglas et al., 2005; Takahashi et al., 2016).

Although both $B A X$ and $B C L 2$ genes show increased expression with time in culture, conclusions on the apoptotic state of COCs cannot be drawn until the ratio of $B A X: B C L 2$ is considered. BAX is a promotor of apoptosis while BCL2 is anti-apoptotic and counters the effects of $B A X$. When considering the ratios of $B A X: B C L 2$, an overall decrease in apoptotic COCs with progression of IVM is a positive indicator for the attainment of oocyte developmental competency and quality. Interestingly, at both 7.5 and 24 hours, the presence of OSFs resulted in low ratios of $B A X: B C L 2$. These results suggest that the addition of OSFs has a protective mechanism against apoptosis and significantly promotes cell survival by reducing the expression of pro-apoptotic genes relative to the ratio of antiapoptotic genes. An interesting effect observed was that the control COCs showed an increase in $B A X: B C L 2$ ratio at 7.5 hours. This suggests that at this particular timepoint, COCs are particularly vulnerable to the microenvironment. This vulnerability is also somewhat reflected in the expression of HIF1A which is increased at 7.5 hours however, as mentioned above this increase is demonstrated across groups and does not always necessarily reflect on the negative implications of oxidative stress until the end of the maturation period. In this sense, $B A X: B C L 2$ is a better biomarker to directly reflect on 
stress and highlights the importance of investigating multiple genes in order to retrieve a more informative and accurate representation of the cell.

By 24 hours, the overall expression of $B A X: B C L 2$ is lower, which is interpreted that the majority of COCs that will undergo apoptosis, will have done so by now and most go on to complete maturation. Interestingly, the high GDF9:BMP15 ratio resulted in reduced $B A X: B C L 2$ expression, relative to the control by this timepoint. This contests previous reports claiming that BMP15 is a key player in reducing apoptosis by downregulating proapoptotic factors such as BAX (Hussein et al., 2005). However, our findings again support that a higher GDF9:BMP15 ratio does result in better quality and more developmentally competent oocytes relative to the other ratio doses, which would support the increased blastocyst rates in sheep which was observed by Clark (2018). It is important to note the low GDF9:BMP15 ratio demonstrates a near significant interaction with the control treatment in which it also showed reduced expression of $B A X: B C L 2$ at 24 hours. This supports the hypothesis that BMP15 plays a key role in reducing apoptosis. With increased statistical power, a clearer interaction could be observed to make more accurate comparisons.

In future, it would be also interesting to compare this data with the number of morphologically-degenerate $\operatorname{COC}$ (Grade 5; Table 3) at this timepoint however, the statistical power from this study was too low to make accurate comparisons.

The COC however, has evolved several mechanisms to combat the detrimental effects of ROS. One of these processes is by increasing the level of antioxidants to detoxify the cell hence, antioxidants can also serve as great biomarkers to assess cell quality. While the ooplasm may face high levels of hypoxia and ROS production, the increase of antioxidants such as GSH and the expression of PRDX6 appear to combat the detrimental effects of this so that the microenvironment is detoxified and favourable.

Overall, this study found that GSH:MCB conjugation levels increased significantly at each of the timepoints tested. Conjugation levels are relative to the level of intracellular GSH within the oocyte which is indicative of the overall oxidative state. High levels of GSH and other antioxidants within the $\mathrm{COC}$ are known biomarkers of improved developmental competency and a positive influence on the completion of oocyte cytoplasmic maturation, 
and fertilisation. At 7.5 hours, GSH levels were highest in oocytes incubated in IVM media supplemented with a high GDF9:BMP15 ratio relative to all other treatment and control groups. Moreover, oocytes incubated in a 1:1 GDF9:BMP15 ratio exhibited higher GSH levels, relative to the low GDF9:BMP15 ratio and control groups. However, by 24 hours there is no difference in GSH levels regardless of treatment which suggests that the level of GSH within the $\mathrm{COC}$ reaches a saturation point as maturational events come to a completion. Interestingly, the difference in GSH levels for the high GDF9:BMP15 ratio does not appear to differ between the two incubation times suggesting this optimal level is reached at a much earlier point over the maturation period. This appears to be due to the influence of high levels of GDF9, suggesting that oocytes incubated in an IVM system supplemented with a high concentration of GDF9 will reach a more favourable environment much earlier in the maturation period, than all other treatments investigated. A detoxified environment with minimal presence of ROS would be extremely beneficial for an oocyte to attain developmental competency which positively correlates with previous findings where the supplementation of IVM media with a high GDF9:BMP15 ratio leads to increased blastocyst rates in sheep (Clark, 2018). Previous studies by Sudiman et al. (2014) have reported that BMP15, both in the presence and absence of $\mathrm{FSH}$, resulted in increased GSH levels in bovine oocytes relative to GDF9. While our results contest this, it does highlight the synergistic effects of OSFs when supplemented together.

Despite these promising results, it is important to note that this assay has its redundancies. Firstly, this assay relies on the conjugation of MCB to GSH; a reaction that is catalysed by the GST family of enzymes. This family of enzymes are functionally and structurally similar however, do have different specificities to MCB (Cook et al., 1991; Ublacker et al., 1991). Thus, different conjugations rates, and even incomplete levels of conjugation, due to the heterogeneous nature of the enzymes may impact fluorescent readings. More importantly, while it is heavily reported that increased levels of GSH are a positive biomarker for improved developmental competency, maturation and oocyte quality, the entire oxidative state of the $\operatorname{COC}$ must be considered. For this assay in particular, the ratio of GSH:GSSG (reduced:oxidised glutathione) must be taken into account before conclusive statements about the oxidative state of a cell can be made. While we have optimised this technique to measure GSH levels in the COC, the assay 
needs to be further optimised to measure, compare and contrast GSSG levels relative to GSH at each timepoint over the incubation period.

To combat the redundancies of the assay, the expression levels of other antioxidant biomarkers can also be used to determine the rate of detoxification levels within the COC. In this study, expression levels of PRDX6, SOD1 and GSTA1 were investigated. The levels of oocyte-derived PRDX6 mRNA, regardless of treatment, were elevated at 7.5 and 24 hours, compared to 1 hour, of culture. This gene encodes a group of antioxidant enzymes that facilitate detoxification of the cell. Its increase in expression as maturation progresses is a positive indication of overall oxidative state and developmental competency (Leyens et al., 2004). In contrast, there were no changes in the expression levels of SOD1 mRNA in the oocytes. However, this was expected as it is known that the CC tend to have a protective mechanism against alterations in the molecular events that occur in the oocyte (Campen et al., 2017), which is also reflected in the expression levels of BMP15 and GDF9.

In this study, GSTA1 mRNA was progressively downregulated at 7.5 hours, regardless of treatment. These results are directly concomitant to previous reports by Salhab et al. (2011) who found that the expression and protein abundance levels of GSTA1 decreased transiently over the course of IVM in gonadotropin and growth factor-enriched media. GSTA1 is an enzyme involved in the regulation of the oxidative stress response. It detoxifies the cell of electrophilic compounds and products through the conjugation of GSH. The alpha class of GST enzymes specifically exhibits peroxidase activity and thereby protect cells from ROS and peroxidation (Salhab et al., 2013). Its molecular profile is associated with steroidogenically-active cells and is heavily regulated by the presence of gonadotropins (Rabahi et al., 1999). Both of these studies suggest that GSTA1 is linked to ROS production during steroid synthesis and high metabolism, where the absence of gonadotropins and growth factors induce higher levels of ROS. From this information and our results, it is speculated that the decrease in GSTA1 levels is concomitant to a decrease in ROS. If true, GSTA1 may work as a "stress-sensing" mechanism, and is likely to be extremely beneficial for the completion of cytoplasmic maturation by 24 hours, one of the biggest limitations facing the success rate of IVM. As the cellular microenvironment becomes more favourable, there is less of a need for GSTA1. 
However, the growth factor and gonadotrophin-enriched media in the studies conducted by Salhab et al. (2011) did not include BMP15 and GDF9. In relation to our results, this highlights that although OSFs may not necessarily play a role in the expression levels of GSTA1, they may nevertheless improve the oxidative state of the cell through the increased detoxification of the cell by promoting GSH and PRDX6 at 7.5 hours. When relating these positive biomarkers to oxidative stress indicators such as HIF1A, it appears that oxidative stress, likely due to the presence of ROS as a result of increased oxidative and carbohydrate metabolism, is high at 7.5 hours. This is when the $\mathrm{COC}$ will be undergoing dynamic changes and adapting to the conditions of the IVM system. By 24 hours, oxidative stress is decreased due to the increased presence of antioxidants such as GSH and PRDX6. While no treatment effects were observed in contrast to the GSH assay, it is important to highlight that GSTA1 mRNA levels in this instance were measured in the CC, whereas GSH levels were measured within the oocyte. It would be interesting to see whether investigating expression levels of GSTA1 mRNA within the oocyte would show treatment effects that correlate to the outcomes presented by the GSH assay. 


\subsection{Effect of GDF9 \& BMP15 on Nuclear Maturation Networks}

Events associated with cytoplasmic maturation often do not occur without the supplementation of correct pre-IVM factors, such as C-type natriuretic peptide (CNP), to prevent the event of spontaneous nuclear maturation when the $\mathrm{COC}$ is removed from the molecular environment and placed into a culture dish with media deficiencies. When a $\mathrm{COC}$ is removed from the follicle, GJ networks are disrupted but can re-establish, as reflected in our initial GJ time series experiments. Specifically, GJ activity was low immediately after extraction from the oocyte (Time $1 \mathrm{hr}$ ) but was increased by 4 hours in incubation. For those COCs where GJ remain disorganised, spontaneous nuclear maturation events are likely to occur. Concomitant to other studies, GJ are reduced over the course of IVM in culture systems, with no dynamic changes in GJ activity after $7.5 \mathrm{~h}$ (Thomas et al., 2004; Ward, 2018). A decrease in GJ activity corresponds to the closure of GJs throughout the $\mathrm{COC}$, and is an indicator of GVBD and meiotic resumption suggesting completion of nuclear maturation events. Interestingly, relative to bovine and porcine IVM systems, GJ activity reaches peak levels earlier at 6-10 hours and 12-18 hours, respectively. This suggests that there could be a species difference in GJ communication throughout the COC (Sasseville et al., 2009). However, it is important to note that the decrease in GJ communication after 7.5 hours in culture was to be expected as this is where, in the ovine biphasic system, a change from Early to Late media occurs. Our "Late" media contains hCG which mimics the ovulation signal that would induce GC luteinisation in the whole follicle and close GJs. While some COC are expected to spontaneously close the GJs as a response to $\mathrm{hCG}$, others would gradually respond to the luteinisation signal for the remainder of the culture time. Therefore, it is crucial to recognise that the direct effects and responses are also heavily influenced by which factors are supplemented into the media as well as how many media phases are present within the IVM system. As reflected in the results investigating oxidative stress, it is implied that the COC is particularly vulnerable during this time.

When investigating the effects of the different ratio doses on GJ communication, it was demonstrated that the supplementation of IVM media with the highest ratio of GDF9:BMP15 significantly improved GJ activity relative to the low GDF9:BMP15 treatments. These results suggest that the addition of a high GDF9:BMP15 ratio prolongs 
the closure of GJs in a biphasic IVM system hence, allowing the arrested state of the oocyte to extend over a longer period of time. Under this meiotically-arrested condition, the preservation of communication within the COC by maintaining GJ activity theoretically allows for additional time to complete events associated with cytoplasmic maturation such as the molecular transport of glycolytic carbohydrates, pro-apoptotic factors and antioxidants to combat oxidative stress and aid in cell survival. These results positively correlate with previous findings in the Pitman lab in which the supplementation of IVM media with a high GDF9:BMP15 ratio also leads to increased blastocyst rates in sheep (Clark, 2018).

CX43 is a GJ protein, particularly between CC, which has been implicated in the attainment of developmental competence. In this study, the expression of $C X 43$ mRNA was higher in the later stages of the IVM period (at 7.5 and 24 hours). Interestingly at 24 hours, the higher GDF9:BMP15 ratio demonstrated greater expression levels of $C X 43$ mRNA, relative to the control. This is in contrast to the expected decrease reflective of the closure of GJ as maturation is completed. While this is a promising result as it correlates with our GJ assay results at 24 hours, it has been reported in literature that the poorly timed expression of particular genes, such as $C X 43$, can have negative implications on the formation of embryos. Studies by Hasegawa et al. (2007) demonstrated that while the fertilisation and cleavage rates of embryos resulting from human oocytes with high CX43 expression levels at the end of maturation was not compromised, blastocyst cell quality was higher in embryos that have diminished $C X 43$ in their CC prior to fertilisation via intracytoplasmic sperm injection (ICSI). Feuerstein et al. (2007) also reported similar results where oocytes with higher transcript levels of $C X 43$ in their CC were less likely to develop beyond the embryo stage. While our results contradict these findings, other studies have demonstrated that GJ communication in porcine COC at 24 hours is no longer associated with CX43 proteins but rather GVBD and compartmentalisation of CX43 into lipid rafts which is heavily dependent on gonadotropins (Sasseville et al., 2009) Hence, only the $C X 43$ expression levels at 7.5 hour were thought to reflect GJ proteins, and the sustained levels of $C X 43$ mRNA are independent of GJ communication. The only way to truly determine whether high expression levels on $C X 43$ have negative implications on embryo development is to conduct further studies investigating the outcomes of 
blastocyst rate and quality from these oocytes. The addition of OSFs at different ratio doses may improve blastocyst rates, though upon further interrogation, the cell quality of these blastocysts may be poor. 


\subsection{Effect of GDF9 \& BMP15 on the Completion of Maturation Events}

As expected, the proportion of oocytes that had undergone GVBD increased as the IVM period progressed, with significant differences in GVBD rates between 1 and 24 hours. This is reflective of the addition of CNP in the "Early" media in the pre-IVM step. GVBD is indicative of meiotic resumption and the completion of nuclear maturation events. However, no differences in GVBD rate between treatment groups were observed suggesting that OSFs do not play a key role in the progression of meiotic resumption.

The degree of CC expansion also increased with IVM progression as expected, particularly towards the latter end of the incubation period as maturational events come to a completion. Upon further interrogation, it was found that there were no differences between treatment groups on the degree of CC expansion. This is further supported by changes in TNFAIP6 transcripts in the CCs, which increases at 24 hours with no differences between treatment groups. TNFAIP6 is a binding protein involved in the formation of the ECM and involved in CC expansion by stabilising the hyaluronan backbone (Fulop et al., 1997). Therefore, OSFs may not be a major contributor to CC expansion but rather, other factors added to IVM media. It is important to note that while CC expansion is an important biomarker of maturation in vivo, it is not reliable for the attainment of developmental competency for in vitro systems, but may indicate IVM media deficiencies.

However, it is important to note that whilst sample sizes for each replicate for each treatment group and timepoint was initially high, the process of mechanical denudation of $\mathrm{COC}$ required removing CC from the oocyte to measure fluorescence and accurately visualise GV, is a rigorous process that often resulted in the rupture of a large number of oocytes. Further refinements need to be made by either increasing sampling size per treatment group and time or by changing the way in which CC are removed. An alternative method could be with the use of hyaluronidase, an enzyme which digests hyaluronic acid interspaced between CC (Moura et al., 2017). This could allow enough statistical power to determine whether the presence of OSFs do in fact influence the timing of GVBD, and maintenance of meiotic resumption, in IVM systems.

Sample sizes were also reduced as CC grades of 4 (including denuded oocytes, or oocytes surrounded only by corona radiata cells) or 5 (degenerate oocytes) were excluded from the dataset (Table 3). Though each COC is variable, the status of CC change over the course 
of maturation due to expansion, oocyte quality, level of bidirectional communication and influences of secreted factors such as OSFs. However, the mechanical transfer from an Early to Late phase medium involved in biphasic IVM systems, while gentle, can disrupt communication within the COC due to the closure of GJ (Ward, 2018) and in some instances, result in detachment of the CC mass from the oocyte. While some COC can recover from this disruption, many will spontaneously undergo premature nuclear maturation.

The changes in the expression levels of key signalling receptors such as $P R, F S H R$ and EGFR genes also reflects the completion of maturation events. In our study, $P R$ mRNA increased in expression levels at 24 hours, with no treatment differences. PR is involved in the regulation of the rupture of the ovarian follicle to release the $\mathrm{COC}$, and is a response to the preovulatory-LH surge. Its expression is transiently increased by LH, forskolin and hCG (Robker et al., 2009). The expression of $P R$ is crucial for the maintenance of pregnancy. The premature timing of $P R$ transcripts has also been associated with poor embryo development (Hasegawa et al., 2005).

Expression of the FSHR gene was highest after 7.5 hours in culture and as expected, its pattern of expression was not influenced by treatment. In vivo, follicular development and many molecular events within the $\mathrm{COC}$ occur as a response to FSH and the cross-talk of other endocrine and paracrine factors like OSFs. This includes follicular growth, the maintenance of meiotic arrest by producing CNP as well as inducing the production of EGFs to promote the functionality of EGFR. However, as maturation progresses and the follicle relies more on $\mathrm{LH}$, or in the case of the biphasic IVM system, the addition of hCG to media which acts in the same fashion as $\mathrm{LH}$, the $\mathrm{COC}$ becomes less responsive to FSH and as a result, FSHR is downregulated throughout the complex.

Unexpectedly, the expression levels of EGFR did not show any changes due to incubation time. However, the presence of OSF upregulated the expression of EGFR mRNA at all time points. The expression of EGFR and the functionality of the translated receptor is heavily promoted by cAMP, EGFs, BMP15 and GDF9 (Sugimura et al., 2015). Without the presence of GDF9 and BMP15, or when OSFs are present at low concentrations, EGFR is not expressed which is also reflected within the results of this study. It is likely that an increase of EGFR levels was not observed with time as EGFs were not supplemented into 
the IVM media. In vivo, EGFs are normally produced and secreted by GC. Some studies have suggested that $\mathrm{CC}$ in murine models potentially undergo morphological changes, possibly reversion, in vitro which cause them to become functionally similar to GC and presumably produce and secrete EGFs (Yang et al., 2016). In future IVM studies, EGFs should be supplemented into IVM media, particularly in the Late phase media where they play a crucial role in the functionality of EGFR and the completion of nuclear maturational events.

The large variability in the data may be masking differences for many of these gene candidates. However, as some of the reported interactions were non-significant, an increase in sample size in future studies may improve robustness and indicate statistical differences. 


\subsection{Effects of GDF9 \& BMP15 on GC Proliferation}

GC proliferation is important for follicular growth and initiated by the cross-talk of GDF9 and BMP15 throughout the ovarian follicle. In vivo, OSFs allow GC to synthesise and secrete important endocrine and paracrine factors such as CNP, oestradiol and EGFs which play a crucial role in the signalling mechanisms of nuclear maturation.

As expected, GDF9 and BMP15 stimulated the proliferation of ovine GC relative to the control. Unexpectedly, there were no differences in the rate of proliferation between the GDF9:BMP15 ratio treatments. There are many reasons why this could have occurred. Firstly, Mottershead et al. (2015) demonstrated that recombinant forms of BMP15 and GDF9 alone do not initiate GC proliferation, therefore whether one OSF is preferably better than the other at stimulating proliferation is quite unlikely considering their synergistic fashion. This assay also demonstrated its redundancies due to the use of inhouse recombinant stocks of porcine GDF9 and BMP15. Though similarities in Ovis aries and Sus scrofa GDF9 and BMP15 gene sequences are high $(87.66 \%$ and $90.11 \%$, respectively), the slight sequence differences may impede the true physiological responsiveness to ovine GCs.

Moreover, assay results from GC display high levels of variability with the dissected population being highly heterogenous due to extraction from follicles at different developmental stages. The divergent morphological and functional properties of GC are dependent on their location within the follicle i.e. whether they are mural or antral or CC (which differentiate from GC) (Salhab et al., 2011; Salhab et al., 2013). Sheep GCs generally exhibit with low viability. Whilst this assay normalises for viability and place a consistent concentration of live cells into each well for the assay, these procedures are imprecise. 


\subsection{Study Limitations}

There were a few limitations encountered whilst conducting these experiments. Firstly, most of these assays produced variable data. While this may be improved by increasing sample size, it should also be noted that there is considerable variability within the COC itself. As follicles are activated to grow one at a time, every growing follicle within an ovary is in a different development state. Thus every COC collected from an ovary has been taken from growing follicles that are at different developmental stages. The collection of COCs followed a strict follicle selection criteria in order to minimise this. However, there will be a proportion of $\mathrm{COC}$ that do not respond to the regulatory agents within the media such as CNP and will therefore undergo spontaneous nuclear maturation and meiotic resumption. Additionally, a corpus luteum or large dominant follicle present on the ovaries from which the follicles are extracted can indicate that an atretic signal has been initiated to all the other antral follicles within that ovary. Whilst late stage atresia in COCs may be distinguished by COC morphology, early atretic changes are often difficult to detect. The ovaries used in these studies were obtained from the local abattoir, and as such, the background of the ewe (e.g. breed, age and health status) was unknown. Also seasonal variability must be taken into account as this can have a large effect on ovarian quality and the number of ovaries collected on any given day. Sheep are seasonal breeders. Whilst most of these experiments were conducted between the peak breeding season (months of May-October, New Zealand), I had little control over the supply of ewe versus lamb ovaries, particularly towards the latter end of the breeding season. Lambs are sub-fertile thus COCs collected were also generally unresponsive to agents in the media. Often times, deficient numbers of COCs were retrieved and numbers within treatment groups had to be reduced. Many of these factors that contribute to variability within the dataset were unfortunately unavoidable. This also affected the numbers of samples left after the denudation and CC grading process.

Similarly, primary cells collected for the GC proliferation assay also showed high variability. It is also important to note that sheep GC in particular generally present with low viability which resulted in large variation between replicates. There are also several methodology issues involved in the GC proliferation assay which could use improvement. 
Another issue is that the physiological ratios of pro-mature:mature forms of OSFs likely have a great effect on the results of these experiments, and ratios present in the recombinant porcine preparations may not reflect that in ovine follicular fluid. Quantifying proteins in follicular fluid has proven challenging due to the high albumin concentrations present (Ward, unpublished results). Stripping albumin from follicular fluid can retain most of the OSFs however, will still inevitably result in a loss of some conjugated proteins including BMP15 and GDF9. Therefore, we can never truly find the physiological ratios of pro-mature and mature forms of OSFs unless better methods are developed.

\subsection{Future Directions \& Learning Steps}

It would be interesting to investigate the effects of the ratios tested in these experiments on embryo development, particularly on blastocyst rate and quality, and associated genes. Though previous studies in the Pitman lab have already demonstrated that higher GDF9:BMP15 ratios did indeed improve blastocyst rates in sheep (Clark, 2018), these results were reported from a single-phase IVM system. Thus, it cannot be guaranteed that these same results would be observed in a biphasic IVM system. Some studies have suggested that phase-changing media has a detrimental impact on COC development. GV oocytes tend to be more membrane stable to environmental stresses than maturing and mature oocytes. Maturing oocytes are so vulnerable to environmental changes that small deviations in their environment can cause meiotic spindles to dissociate which can result in aneuploidy due to the loss of chromosomes (Son et al., 2019). Thus, being able to compare and contrast blastocyst rate and quality between the two systems would be interesting.

While intra-oocyte GSH levels did increase over the IVM period, which is generally associated with developmental competency, a conclusive statement cannot be made about the overall oxidative state of these oocytes until the GSSG:GSH ratio is considered. Furthermore, changes in key metabolites involved in glucose consumption and lactate production by the $\mathrm{COC}$ needs to be further investigated using protocols described by Guerif et al. (2013) and McKeegan (2015). However, it is also important to consider the 
changes in metabolism due to other substrates such as amino acids and lipid droplets within the COC. Both the detection of amino acids as well as observation and staining of lipid droplets has been well established in literature.

The Pitman lab over the past few years has created two novel physiologically-relevant bovine IVM medias which are intended to mimic a good and poor follicular environment of the lactating dairy cow (Murray, 2020). The "Good" media demonstrated vast improvements in gap junction activity and changes in gene expression that represented improvements in oocyte developmental competency in both the CC and oocyte, relative to the commercial IVM media utilised in this study. Commercial media is not representative of the follicular environment in the dairy cow and has emphasised the importance of using physiologically-relevant systems in vitro to improve the attainment of developmental competency and ART success rates. It would be interesting to examine whether a physiologically-relevant ovine IVM system, with the addition of OSFs at different ratios would result in further improvements relative to the commercial system utilised within this study.

\subsection{Concluding Remarks}

To conclude, the studies reported herein demonstrated that commercial IVM media may be improved by the addition of OSFs, and in particular, a high ratio of GDF9 to BMP15. These results indicate that previous observations in blastocyst rate improvement following OSF exposure in sheep oocytes may be due to alterations in mechanisms critical to oocyte maturation during IVM. This includes sustained GJ communication within the COC during the final stages of incubation (i.e. 24 hours). Whilst a decrease of GJ activity was observed by 24 hours, concomitant to other studies, the high GDF9:BMP15 ratio treatment elicited greater GJ activity relative to the low GDF9:BMP15 ratios. This suggests that OSFs may improve the effectiveness of CNP in delaying meiotic resumption. Allowing GJs to remain open longer would allow the accumulation of beneficial metabolites and antioxidants such as pyruvate and GSH. These are crucial to the completion of cytoplasmic maturation and preparation for fertilisation and subsequent embryonic events and were particularly upregulated by the high GDF9:BMP15 ratio. This would also allow sufficient time for the detoxification of the cell, particularly during dynamic phases where the COC 
is metabolically active and likely to be producing high levels of ROS. It is during these phases where the COC appears to be most sensitive to its microenvironment. This is supported by the results of $H I F 1 A$ and $B A X: B C L 2$ expression. From these results, I report that the addition of OSFs regardless of ratio, decreased the susceptibility of the COC to undergo apoptosis. It may be assumed that the maturation events were concluded by 24 hours in IVM culture due to increased expression of EGFR though the addition of OSFs, as well as the upregulation of $P R$ and TNFAIP6 and downregulation of FSHR. These results highlight the importance of maintaining follicular cross-talk and that addition of OSFs, particularly at a high GDF9:BMP15 ratio does significantly improve the gold-standard IVM method. This reflects on the successful fertility rates and high litter sizes of poly-ovulatory mammals. However, OSFs alone do not guarantee the successful development of a blastocyst. Ultimately, the assessment of oocyte maturation and attainment of developmental competency is extremely complex and is the sum of multiple factors. Moreover, the level of bidirectional communication and cross-talk between entire molecular signalling systems within and between all follicular cell types need to be considered to determine the overall quality of the COC. However, with further analyses, these results suggest there is potential that the addition of high GDF9:BMP15 ratio to gold-standard IVM systems can produce more developmentally competent oocytes and high quality embryos for low fecundity mammalian species in a clinical or agricultural setting. 


\section{CHAPTER 5: APPENDICES}

\section{Appendix 1: Western Blot for BMP15 and GDF9}

Various batches of in-house secreted media from HEK-293 cells transfected with plasmids containing $P B M P 15$ or PGDF9, were available for utilisation within this study and have been previously characterised (Alhussini, 2016; Swinerd, 2016). In order to test similarities between batches, a selection of pBMP15 and pGDF9 were analysed through a Western Blot using methods described by Alhussini (2016) and Swinerd (2016). The samples pBMP15 samples analysed were VUW020 C, VUW020 D, VUW020 E, NZ029, NZ031 and NZ033. The pGDF9 samples tested were VU017 B and VU017 D (Figures 30-31).

The data was standardised by measuring the background which was then subtracted from the band readings and normalised by correcting against the measurement of the positive control (VUW020 C, $0.94 \mathrm{ng} / \mathrm{mL}$; VU017 B $0.58 \mathrm{ng} / \mathrm{mL}$ ) on ImageStudio Lite Software for gel analysis (Li-COR Biosciences). However, for increased accuracy, statistical power and robustness, these need to be repeated and averaged. 


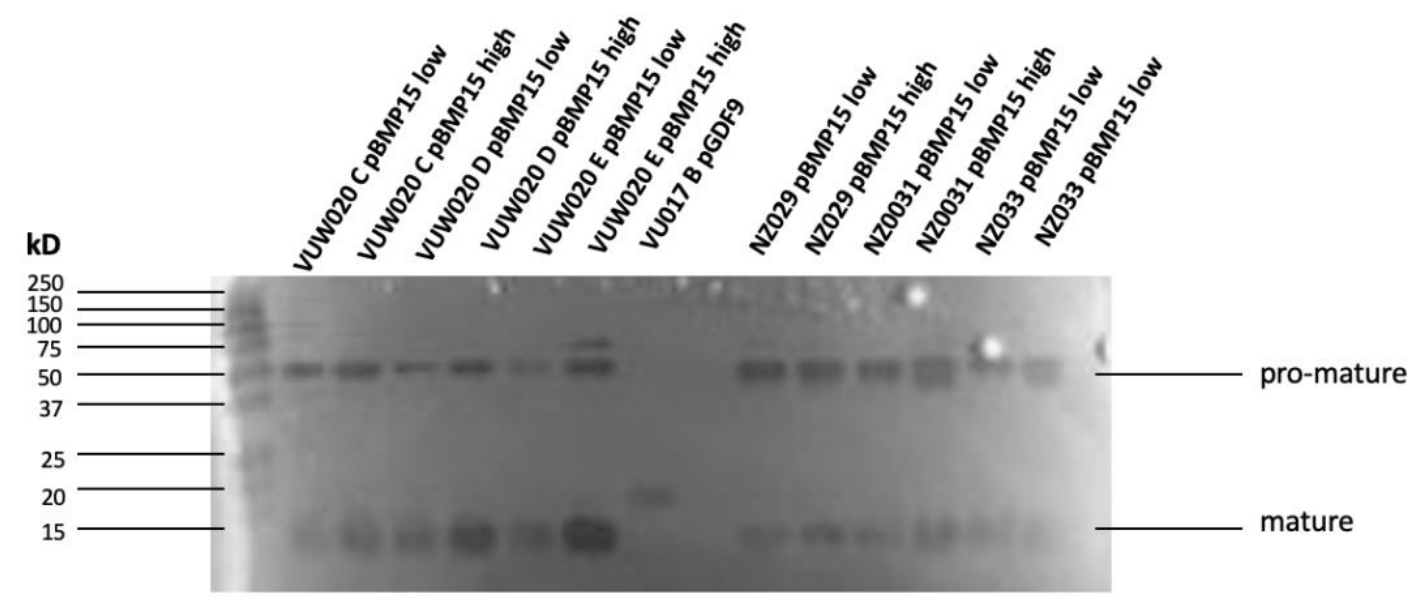

Figure 30: Western Blot of different batches of in house pBMP15.

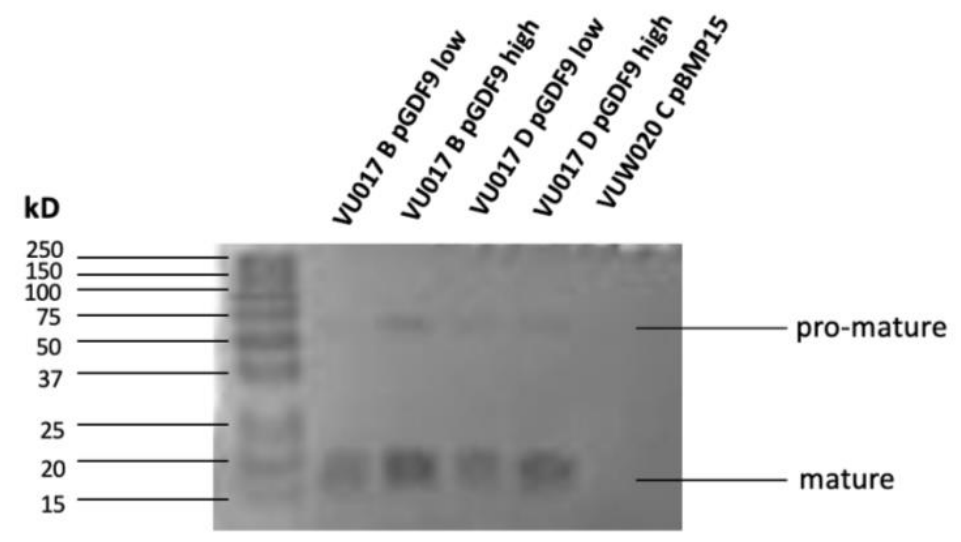

Figure 31: Western Blot of different batches of in house pGDF9. 


\section{Appendix 2: Stocks, Solutions \& Media Recipes}

\section{Dissection \& IVM}

\begin{tabular}{ll}
\hline $\mathbf{0 . 9 \%}$ SALINE & \\
\hline $\mathrm{NaCl}$ & $9 \mathrm{~g}$ \\
\hline $\mathrm{dH}_{2} \mathrm{O}$ & $1 \mathrm{~L}$ \\
\hline & \\
\hline $\mathbf{0 . 7 \%}$ ETHANOL & $700 \mathrm{~mL}$ \\
\hline $100 \%$ denatured Ethanol & $300 \mathrm{~mL}$ \\
\hline $\mathrm{dH} \mathrm{H}_{2} \mathrm{O}$ & \\
\hline
\end{tabular}

\begin{tabular}{ll}
\hline 2X MEDIUM 199 (M199) \\
\hline Osmolality: 490-500 mOsm \\
\hline Medium 199 powder (Gibco 31100-035) & 1 satchet \\
\hline $\mathrm{dH}_{2} \mathrm{O}$ & $500 \mathrm{~mL}$ \\
\hline Kanamycin (Sigma K1377-5G) & $63.35 \mathrm{mg}$
\end{tabular}

\begin{tabular}{ll}
\hline \multicolumn{2}{l}{ 250mM HEPES STOCK (STOCK H) } \\
\hline Osmolality: 370-385 mOsm \\
\hline Hepes Free Acid (Sigma H6147-25G) & $750 \mathrm{mg}$ \\
\hline Hepes Sodium Salt (Sigma H3784-25G) & $812.5 \mathrm{mg}$ \\
\hline $\mathrm{dH}_{2} \mathrm{O}$ & $25 \mathrm{~mL}$
\end{tabular}

\begin{tabular}{ll}
\hline $20 \%$ BOVINE SERUM ALBUMIN (BSA) \\
\hline BSA & $4 \mathrm{~g}$ \\
\hline $0.9 \%$ Saline & $20 \mathrm{~mL}$ \\
\hline
\end{tabular}

\begin{tabular}{ll}
\hline 5000IU/mL HEPARIN (HEPARIN SOLUTION) & \\
\hline Heparin & $23.58 \mathrm{~g}$ \\
\hline $\mathrm{dH}_{2} \mathrm{O}$ & $1 \mathrm{~mL}$
\end{tabular}




\section{H199}

Osmolality: 275-284 mOsm

\begin{tabular}{ll}
\hline $2 X$ M199 Stock & $100 \mathrm{~mL}$ \\
\hline Stock H & $16 \mathrm{~mL}$ \\
\hline $\mathrm{dH}_{2} \mathrm{O}$ & $84 \mathrm{~mL}$
\end{tabular}

\begin{tabular}{ll}
\hline OVARY DISSECTION MEDIA & \\
\hline H199 & $48.5 \mathrm{~mL}$ \\
\hline Heparin Solution & $93 \mu \mathrm{L}$ \\
\hline $20 \%$ BSA & $1 \mathrm{~mL}$
\end{tabular}

\section{COC STORAGE MEDIA}

Dissection Media

$1 \mathrm{~mL}$

CNP

$4.4 \mu \mathrm{L}$

\section{0 mM SODIUM BICARBONATE}

Osmolality: 430-440 mOsm

\begin{tabular}{ll}
\hline $\mathrm{NaHCO}_{3}$ & $525 \mathrm{mg}$ \\
\hline $\mathrm{dH}_{2} \mathrm{O}$ & $5 \mathrm{~mL}$
\end{tabular}

\section{3 mM SODIUM PYRUVATE}

Osmolality: 50-60 mOsm

\begin{tabular}{ll}
\hline Pyruvate & $18 \mathrm{mg}$ \\
\hline $\mathrm{dH}_{2} \mathrm{O}$ & $5 \mathrm{~mL}$
\end{tabular}

\begin{tabular}{ll}
\hline B199 \\
\hline Osmolality: 288-298 mOsm \\
\hline M199 2X Stock & $12.5 \mathrm{~mL}$ \\
\hline Stock B & $2.5 \mathrm{~mL}$ \\
\hline Stock C & $0.15 \mathrm{~mL}$ \\
\hline Milli Q $\mathrm{H}_{2} \mathrm{O}$ & $9.85 \mathrm{~mL}$
\end{tabular}




\begin{tabular}{ll}
\hline 10mM CYSTEAMINE & \\
\hline Cysteamine Hydrochloride & $10 \mathrm{mg}$ \\
\hline B199 & $8.80 \mathrm{~mL}$
\end{tabular}

\section{0iU/mL HUMAN CHORIONIC GONADOTROPIN}

\begin{tabular}{lc}
$5000 I U / m L ~ h C G$ & 1 vial \\
\hline PBS & $714 \mu \mathrm{L}$
\end{tabular}

\begin{tabular}{ll}
\hline C-TYPE NATRIURETIC PEPTIDE STOCK \\
$\begin{array}{ll} \\
\text { CNP } & 1 \text { vial }(0.5 \mathrm{mg}) \\
\mathrm{dH}_{2} \mathrm{O} & 1 \mathrm{~mL}\end{array}$
\end{tabular}

IVM INCUBATION MEDIA
\begin{tabular}{lr}
\hline M199 1X (Gibco 11150-059) & $9 \mathrm{~mL}$ \\
\hline Charcoal-stripped FCS & $1 \mathrm{~mL}$
\end{tabular}

\section{Gap Junction Assays}

\begin{tabular}{ll}
\hline 1mM CALCEIN-AM STOCK & \\
\hline Calcein-AM & $50 \mu \mathrm{g}(1$ vial) \\
\hline DMSO & $50 \mu \mathrm{L}$ \\
\hline
\end{tabular}

\begin{tabular}{lc}
\hline CALCEIN-AM INTERMEDIATE STOCK \\
\hline Calcein-AM Stock & $0.5 \mu \mathrm{L}$ \\
\hline IVM Incubation Media & $25 \mu \mathrm{L}$ \\
\hline
\end{tabular}

\section{Glutathione-Conjugation Assays}

\begin{tabular}{ll}
\hline 10mM MONOCHLOROBIMANE STOCK (MCB) \\
\hline MCB & $2.26 \mathrm{mg}$ \\
\hline DMSO & $1 \mathrm{~mL}$
\end{tabular}




\section{5 $\mu \mathrm{M}$ MONOCHLOROBIMANE INTERMEDIATE STOCK}

\begin{tabular}{ll}
\hline $10 \mathrm{mM} M C B$ & $1 \mu \mathrm{L}$ \\
\hline IVM Incubation Media & $79 \mu \mathrm{L}$
\end{tabular}

\section{Granulosa Cell Proliferation Assay}

\begin{tabular}{ll}
\hline MODIFIED MCCOY'S 5A \\
\hline McCoy's 5A Medium (16600-082) & $500 \mathrm{~mL}$ \\
\hline Penicillin-Streptomycin & $5 \mathrm{~mL}$
\end{tabular}

$30 \mathrm{mg} / \mathrm{mL}$ POLYVINYL ALCOHOL (PVA)

\begin{tabular}{lc}
\hline PVA & $150 \mathrm{mg}$ \\
\hline PBS & $5 \mathrm{~mL}$
\end{tabular}

\section{GRANULOSA CELL INCUBATION MEDIA}

\begin{tabular}{ll}
\hline Modified McCoy's 5A & $49.5 \mathrm{~mL}$ \\
\hline $30 \mathrm{mg} / \mathrm{mL}$ PVA & $0.5 \mathrm{~mL}$
\end{tabular}

Glycolytic Metabolites in IVM Media

\begin{tabular}{ll}
\hline 4.6mM EPPS BUFFER, $\mathbf{p H} 8.0$ \\
\hline EPPS & $16 \mathrm{mg}$ \\
\hline $\mathrm{dH}_{2} \mathrm{O}$ & $100 \mathrm{~mL}$ \\
\hline
\end{tabular}

\begin{tabular}{ll}
\hline 40IU/mL LACTATE DEHYROGENASE (LDH) \\
\hline LDH & $0.036 \mathrm{mg}$ \\
\hline EPPS buffer & $1 \mathrm{~mL}$
\end{tabular}

\section{3mM SODIUM PYRUVATE STANDARD IN PBS}

\begin{tabular}{lc}
\hline Sodium Pyruvate & $28.1 \mathrm{mg}$ \\
\hline PBS & $20 \mathrm{~mL}$
\end{tabular}




\begin{tabular}{lc}
\hline 13mM SODIUM PYRUVATE STANDARD IN DMEM \\
\hline Sodium Pyruvate & $28.1 \mathrm{mg}$ \\
\hline DMEM (11966025) & $20 \mathrm{~mL}$ \\
\hline & \\
\hline 0.1mM NADH & \\
\hline $\mathrm{NADH}$ & $2.84 \mathrm{mg}$ \\
\hline $\mathrm{dH}{ }_{2} \mathrm{O}$ & $20 \mathrm{~mL}$ \\
\hline
\end{tabular}

\section{Western Blot}

\subsection{TRIS HYDROCHLORIDE, pH 6.8 (Tris-HCl)}

\begin{tabular}{ll}
\hline Tris Base & $6.06 \mathrm{~g}$ \\
\hline Milli Q $\mathrm{H}_{2} \mathrm{O}$ & $80 \mathrm{~mL}$ \\
\hline $\mathrm{HCl}$ & To $\mathrm{pH} 6.8$ and add Milli Q $\mathrm{H}_{2} \mathrm{O}$ to $100 \mathrm{~mL}$
\end{tabular}

\begin{tabular}{ll}
\hline 1.5M TRIS HYDROCHLORIDE, $\mathbf{p H ~} \mathbf{8 . 8}$ (Tris-HCl) \\
\begin{tabular}{ll}
\hline Tris Base & $36.34 \mathrm{~g}$ \\
\hline Milli Q $\mathrm{H}_{2} \mathrm{O}$ & $160 \mathrm{~mL}$ \\
\hline $\mathrm{HCl}$ & To $\mathrm{pH} 8.8$ and add Milli Q $\mathrm{H}_{2} \mathrm{O}$ to $200 \mathrm{~mL}$
\end{tabular}
\end{tabular}

\begin{tabular}{ll}
\hline $10 \%$ AMMONIUM PERSULFATE (APS) \\
\hline APS & $1 \mathrm{~g}$ \\
\hline Milli Q $\mathrm{H}_{2} \mathrm{O}$ & $10 \mathrm{~mL}$ \\
\hline
\end{tabular}

\begin{tabular}{ll}
\hline 5X RUNNING BUFFER & \\
\hline Glycine & $360 \mathrm{~g}$ \\
\hline Tris Base & $75 \mathrm{~g}$ \\
\hline SDS & $25 \mathrm{~g}$ \\
\hline
\end{tabular}


1M TRIS HYDROCHLORIDE, pH 8.5 (Tris-HCl)

Tris Base $30.29 \mathrm{~g}$

$\mathrm{HCl}$

To $\mathrm{pH} 8.5$ and add Milli Q $\mathrm{H}_{2} \mathrm{O}$ to $250 \mathrm{~mL}$

\begin{tabular}{ll}
\hline MEMBRANE WASHING BUFFER \\
\hline $1 \mathrm{M}$ Tris-HCl & $20 \mathrm{~mL}$ \\
\hline $\mathrm{NaCl}$ & $8.6 \mathrm{~g}$ \\
\hline Tween $^{\circledR} 20$ & $1 \mathrm{~mL}$ \\
\hline Milli Q $\mathrm{H}_{2} \mathrm{O}$ & Up to $1 \mathrm{~L}$
\end{tabular}

$5 \%(w / v)$ BLOCKING BUFFER

Membrane Washing Buffer $20 \mathrm{~mL}$

Skim Milk Powder $1 \mathrm{~g}$

$10 \%(w / v)$ SODIUM DODECYL SULFATE (SDS)

SDS $2 \mathrm{~g}$

Milli Q $\mathrm{H}_{2} \mathrm{O} \quad 20 \mathrm{~mL}$ 
Appendix 3: Chemicals \& Reagents

\begin{tabular}{|c|c|c|}
\hline Components & Manufacturer & Catalogue Number \\
\hline 2-Mercaptoethanol & Sigma Aldrich, USA & M3148-100mL \\
\hline$\beta$-scintillation fluid & Perkin Elmer & SC/9200.21 \\
\hline $17 \beta$-Oestradiol & Sigma Aldrich, USA & E8875 \\
\hline $30 \%$ Bis-acrylamide & BioRad & Cat\#161-0158 \\
\hline 5000IU Chorion $®$ hCG & Provet NZ Pty Ltd & $\mathrm{A} 2377$ \\
\hline Ammonium Persulfate & BioRad & $161-07000$ \\
\hline $\begin{array}{l}\text { Bovine serum albumin (Low } \\
\text { endotoxin) }\end{array}$ & MP Biomedicals, NZ & 11BSASG100 \\
\hline $\begin{array}{l}\text { Brilliant Multiplex QPCR Master } \\
\text { Mix }\end{array}$ & Agilent Technologies, CA, USA & 600553 \\
\hline Brilliant II SYBR Green & Agilent Technologies, CA, USA & 600828 \\
\hline Calcein-acetoxymethyl & Thermo Fisher Scientific, CA, USA & C3100MP \\
\hline Charcoal & Sigma Aldrich, USA & C-5260 \\
\hline Cysteamine Hydrochloride & Sigma Aldrich, USA & M6500-25G \\
\hline DMSO & Sigma Aldrich, USA & D2650 \\
\hline $\begin{array}{l}\text { DMEM (Glucose and Pyruvate- } \\
\text { free) }\end{array}$ & Gibco Life Technologies & 11966025 \\
\hline EPPS & Sigma Aldrich, USA & E1894-25G \\
\hline Ethanol (RNase-free) & Global Science, NZ & АppA392810000РE \\
\hline Foetal calf serum (NZ origin) & Gibco Life Technologies & 10091130 \\
\hline Glycine & Fisher Scientific & $\mathrm{G} / 0800 / 60$ \\
\hline Heparin & Sigma Aldrich, USA & H3149 \\
\hline Hepes free acid & Sigma Aldrich, USA & $\mathrm{H} 6147$ \\
\hline Hepes sodium salt & Sigma Aldrich, USA & H3784 \\
\hline Kanamycin & Sigma Aldrich, USA & K1377 \\
\hline Lactate Dehydrogenase & Sigma Aldrich, USA & 10127884001 \\
\hline MasterPure ${ }^{\mathrm{TM}}$ RNA Purification Kit & Lucigen & MCR85102 \\
\hline McCoys $5 a$ & Gibco Life Technologies & $16600-082$ \\
\hline M199 Earle's Salts Powder & Gibco Life Technologies & 31100019 \\
\hline M199 1X & Gibco Life Technologies & $11150-059$ \\
\hline M199 1X (Phenol Red-Free) & Gibco Life Technologies & 11043 \\
\hline
\end{tabular}




\begin{tabular}{|c|c|c|}
\hline Components & Manufacturer & Catalogue Number \\
\hline Mineral Oil & Sigma Aldrich & M8410 \\
\hline Monochlorobimane & Invitrogen, Thermo Fisher & 10138023001 \\
\hline NADH & Sigma Aldrich, USA & 10128023008 \\
\hline Natriuretic peptide $\mathrm{C}$ & Sigma Aldrich, USA & N8768 \\
\hline OVA2 (FSH) & \multicolumn{2}{|c|}{ Dr. Jenny Juengel, AgResearch Invermay, NZ } \\
\hline Penicillin-Streptomycin & Gibco Life Technologies & 15140122 \\
\hline Peroxidase-conjugated AffiniPure & Jackson ImmunoResearch & $315-035-045$ \\
\hline $\begin{array}{l}\text { Rabbit Anti-Mouse IgG (Minimal } \\
\text { cross reaction to Human Serum }\end{array}$ & Laboratory Inc, USA & \\
\hline \multicolumn{3}{|l|}{ Proteins) } \\
\hline Polyvinyl Alcohol & Sigma Aldrich, USA & P8136-250G \\
\hline Phosphate buffered saline (PBS) & Gibco Life Technologies & $10010-033$ \\
\hline Precision Plus Protein ${ }^{\mathrm{TM}}$ Dual & BioRad & 1610374 \\
\hline \multicolumn{3}{|l|}{ Colour Standard } \\
\hline Sodium dodecyl sulfate (SDS) & Sigma Aldrich, USA & $74255-250$ \\
\hline Sodium bicarbonate & Sigma Aldrich, USA & S5761 \\
\hline Sodium chloride & ThermoFisher Scientific, CA, USA & S-3160-60 \\
\hline Sodium pyruvate & Sigma Life Science, USA & P4562-5G \\
\hline SuperScript ${ }^{\circledR}$ VILO ${ }^{\text {TM }}$ cDNA & ThermoFisher Scientific, CA, USA & 11754250 \\
\hline \multicolumn{3}{|l|}{ Synthesis Kit } \\
\hline TEMED & Sigma Aldrich, USA & $19281-25 \mathrm{~mL}$ \\
\hline Tris Base & Fisher Scientific, USA & BP152-1 \\
\hline Tritiated Thymidine & Perkin Elmer, Boston, MA, USA & NET335501MC \\
\hline Trypan Blue & Gibco Life Technologies & 15250061 \\
\hline Tween $^{\circledR} 20$ & Sigma Aldrich, USA & BP337-500 \\
\hline UltraPure $^{\mathrm{TM}}$ distilled water & ThermoFisher Scientific, CA, USA & 109977 \\
\hline WesternBright ${ }^{\mathrm{TM}}$-ECL Spray & Advansta & K-12049-D50 \\
\hline
\end{tabular}




\section{Appendix 3: Consumables \& Equipment List}

\section{Consumables}

\begin{tabular}{|c|c|c|}
\hline Consumable Item & Manufacturer & Catalogue Number \\
\hline $15 \mathrm{~mL}$ Centrifuge Tubes & Corning Incorporated, USA & 430791 \\
\hline $50 \mathrm{~mL}$ Centrifuge Tubes & Corning Incorporated, USA & 430829 \\
\hline $0.1 \mathrm{~mL}$ PCR Strip Tubes & Qiagen, Germany & 981103 \\
\hline $0.2 \mathrm{~mL}$ PCR Tubes & Axygen & AXYPCR-02-C \\
\hline Clear microcentrifuge tubes (all sizes) & Axygen & \\
\hline $0.6 \mathrm{~mL}$ amber microcentrifuge tubes & Axygen & AXYMCT-060-C \\
\hline $1.5 \mathrm{~mL}$ amber microcentrifuge tubes & Axygen & AXYMCT-150-C \\
\hline $35 \mathrm{~mm}$ culture dish & Jet Biofil, China & TCD000035 \\
\hline $60 \mathrm{~mm}$ culture dish & Jet Biofil, China & $2303-1060$ \\
\hline $90 \mathrm{~mm}$ culture dish & Jet Biofil, China & 150350 \\
\hline BD Precisionglide ${ }^{\circledR}$ 20G-gauge needle & Sigma Aldrich & 302025 \\
\hline BD $3 \mathrm{~mL}$ rubber-free aspiration syringe & Sigma Aldrich & 302113 \\
\hline BD $60 \mathrm{~mL}$ syringe & Interlab & SG-503P-LL \\
\hline Ministart ${ }^{\circledR}$ syringe filter $(0.2 \mu \mathrm{m})$ & $\begin{array}{l}\text { Sartorius Stedim Biotech, } \\
\text { Germany }\end{array}$ & 16534 \\
\hline $\begin{array}{l}\text { Corning }{ }^{\circledR} \text { Bottle-Top Vaccum Filter and } \\
\text { Bottle }\end{array}$ & Corning Incorporated, USA & CLS430769 \\
\hline Filtered pipette tips (all sizes) & Axygen & \\
\hline $\begin{array}{l}\text { Eclipse Unfiltered pipette tips (all } \\
\text { sizes) }\end{array}$ & Uniware & \\
\hline 96-well plate (untreated) & Jet Biofil, China & TCP-000-096 \\
\hline 96-well plate (delta treated) & NUNC, Themo Fisher & NUN167008 \\
\hline $\begin{array}{l}\text { 96-well plate for fluorescence-based } \\
\text { assays }\end{array}$ & Invitrogen & M33089 \\
\hline 1450 micro fibre filter $90 \times 20$ filter mat & Perkin Elmer, MA, USA & $1450-421$ \\
\hline Sample bag & Perkin Elmer, MA, USA & $1450-432$ \\
\hline
\end{tabular}


Specialised Equipment \& Software

\begin{tabular}{ll}
\hline \multicolumn{1}{c}{ Equipment \& Software } & \multicolumn{1}{c}{ Manufacturer } \\
\hline Beacon Designer Software & Premier Biosoft International, CA, USA \\
\hline ImageJ Software & U.S National Instittute of Health \\
\hline ImageStudio Lite & Li-COR Biosciences \\
\hline Prism Software 8 & GraphPad \\
\hline Rotor-Gene ${ }^{\text {TM }}$ Rotary Analyzer (11754-050) & Qiagen, Germany \\
\hline Dissection Microscope & Leica Microsystems, Germany \\
\hline CRAIC Inverted Microscope, CTR4000B & Leica Micosystems, Germany \\
\hline CRAIC CCD Image Software & Leica Micosystems, Germany \\
\hline Haemocytometer & Improved Neubauer, BS 74B, Hawksley \\
\hline Omega Lum-G Imaging System & Aplengen \\
\hline Wallac Cell Harvester & TOMTEC \\
\hline Wallac micro trillux scintillation counter & Perkin Elmer \\
1450 LSC \& Luminescence Counter & \\
\hline CLARIOstar ${ }^{\circledR}$ Plus Microplate Reader & BMG Labtech \\
\hline
\end{tabular}




\section{REFERENCES}

Abe, H., Yamashita, S., Itoh, T., Satoh, T., \& Hoshi, H. (1999). Ultrastructure of bovine embryos developed from in vitro-matured and -fertilized oocytes: Comparative morphological evaluation of embryos cultured either in serum-free medium or in serum-supplemented medium. Molecular Reproduction and Development, 53(3), 325-335.

Albuz, F. K., Sasseville, M., Lane, M., Armstrong, D. T., Thompson, J. G., \& Gilchrist, R. B. (2010). Simulated physiological oocyte maturation (SPOM): a novel in vitro maturation system that substantially improves embryo yield and pregnancy outcomes. Hum Reprod, 25(12), 2999-3011.

Alhussini, A. A. (2016). The molecular forms of GDF9 in a range of mammalian species (Master's thesis). Victoria University of Wellington.

Amsterdam, A., Koch, Y., Lieberman, M. E., \& Lindner, H. R. (1975). Distribution of binding sites for human chorionic gonadotropin in the preovulatory follicle of the rat. J Cell Biol, 67(3), 894-900.

Aquilano, K., Baldelli, S., \& Ciriolo, M. R. (2014). Glutathione: new roles in redox signaling for an old antioxidant. Frontiers in Pharmacology, 5.

Baby, T. E., \& Bartlewski, P. M. (2011). Circulating concentrations of ovarian steroids and follicle-stimulating hormone (FSH) in ewes with 3 or 4 waves of antral follicle emergence per estrous cycle. Reproductive Biology, 11(1), 19-36.

Barbosa, L. F., Cerqueira, F. M., Macedo, A. F. A., Garcia, C. C. M., Angeli, J. P. F., Schumacher, R. I., ... Medeiros, M. H. G. (2010). Increased SOD1 association with chromatin, DNA damage, p53 activation, and apoptosis in a cellular model of SOD1-linked ALS. Biochimica Et Biophysica Acta-Molecular Basis of Disease, 1802(5), 462-471.

Bartlewski, P. M., Baby, T. E., \& Giffin, J. L. (2011). Reproductive cycles in sheep. Animal Reproduction Science, 124(3-4), 259-268.

Belkadi, S., Safsaf, B., Heleili, N., Tlidjane, M., Belkacem, L., \& Oucheriah, Y. (2017). Seasonal influence on sperm parameters, scrotal measurements, and serum testosterone in Ouled Djellal breed rams in Algeria. Vet World, 10(12), 1486-1492.

Biggers, J. D., Whittingham, D. G., \& Donahue, R. P. (1967). The pattern of energy metabolism in the mouse oocyte and zygote. Proc Natl Acad Sci U S A, 58(2), 560-567. 
Blondin, P., \& Sirard, M. A. (1995). Oocyte and follicular morphology as determining characteristics for developmental competence in bovine oocytes. Mol Reprod Dev, 41(1), 54-62.

Bracken, C. J., Radcliff, R. P., McCormack, B. L., Keisler, D. H., \& Lucy, M. C. (2007). Two days of pulsatile GnRH infusion beginning 4 days before weaning in sows initiates a wave of follicular growth that is not sustained after weaning. Animal Reproduction Science, 102(1-2), 158-164.

Brinster, R. L. (1969). Radioactive carbon dioxide production from pyruvate and lactate by the preimplantation rabbit embryo. Exp Cell Res, 54(2), 205-209.

Bruzzone, R., White, T. W., \& Goodenough, D. A. (1996). The cellular Internet: on-line with connexins. Bioessays, 18(9), 709-718. doi:10.1002/bies.950180906

Buckett, W. M., Chian, R. C., Holzer, H., Dean, N., Usher, R., \& Tan, S. L. (2007). Obstetric outcomes and congenital abnormalities after in vitro maturation, in vitro fertilization, and intracytoplasmic sperm injection. Obstetrics and Gynecology, 110(4), 885-891.

Bunel, A., Nivet, A. L., Blondin, P., Vigneault, C., Richard, F. J., \& Sirard, M. A. (2014). Cumulus cell gene expression associated with pre-ovulatory acquisition of developmental competence in bovine oocytes. Reprod Fertil Dev, 26(6), 855-865.

Caixeta, E. S., Machado, M. F., Ripamonte, P., Price, C., \& Buratini, J. (2013). Effects of FSH on the expression of receptors for oocyte-secreted factors and members of the EGF-like family during in vitro maturation in cattle. Reproduction Fertility and Development, 25(6), 890-899.

Campen, K. A. (2013). Investigation of exposure to lifestyle and environmental factors on cumulus-oocyte complex function (Ph.D thesis). Victoria University of Wellington.

Carabatsos, M. J., Elvin, J., Matzuk, M. M., \& Albertini, D. F. (1998). Characterization of oocyte and follicle development in growth differentiation factor-9-deficient mice. Dev Biol, 204(2), 373-384.

Cetica, P., Pintos, L., Dalvit, G., \& Beconi, M. (2002). Activity of key enzymes involved in glucose and triglyceride catabolism during bovine oocyte maturation in vitro. Reproduction, 124(5), 675-681.

Cetica, P. D., Pintos, L. N., Dalvit, G. C., \& Beconi, M. T. (1999). Effect of lactate dehydrogenase activity and isoenzyme localization in bovine oocytes and utilization of oxidative substrates on in vitro maturation. Theriogenology, 51(3), 541-550. 
Cha, K. Y., Choi, D. H., Koo, J. J., Han, S. Y., Ko, J. J., \& Yoon, T. K. (1991). Pregnancy after Invitro Fertilization of Human Follicular Oocytes Collected from Nonstimulated Cycles, Their Culture Invitro and Their Transfer in a Donor Oocyte Program. Fertility and Sterility, 55(1), 109-113.

Cha, K. Y., Do, B. R., Chi, H. J., Yoon, T. K., Choi, D. H., Koo, J. J., \& Ko, J. J. (1992). Viability of Human Follicular Oocytes Collected from Unstimulated Ovaries and Matured and Fertilized Invitro. Reproduction Fertility and Development, 4(6), 695-701.

Chang, H., Brown, C. W., \& Matzuk, M. M. (2002). Genetic analysis of the mammalian transforming growth factor-beta superfamily. Endocr Rev, 23(6), 787-823.

Chiquoine, A. D. (1954). The Identification, Origin, and Migration of the Primordial Germ Cells in the Mouse Embryo. Anatomical Record, 118(2), 135-146.

Cho, W. K., Stern, S., \& Biggers, J. D. (1974). Inhibitory effect of dibutyryl cAMP on mouse oocyte maturation in vitro. J Exp Zool, 187(3), 383-386.

Christoforou, E. R. (2017). The effects of oocytes on Smad signalling pathways in granulosa cells (Master's thesis). Victoria University of Wellington.

Clark, Z. L. (2018). Investigating the intrafollicular factors affecting oocyte developmental competency (Ph.D. thesis). Victoria Universiy of Wellington. .

Combelles, C. M., Holick, E. A., Paolella, L. J., Walker, D. C., \& Wu, Q. (2010). Profiling of superoxide dismutase isoenzymes in compartments of the developing bovine antral follicles. Reproduction, 139(5), 871-881.

Combelles, C. M. H., Gupta, S., \& Agarwal, A. (2009). Could oxidative stress influence the in-vitro maturation of oocytes? Reproductive Biomedicine Online, 18(6), 864-880.

Cook, J. A., lype, S. N., \& Mitchell, J. B. (1991). Differential specificity of monochlorobimane for isozymes of human and rodent glutathione S-transferases. Cancer Res, 51(6), 1606-1612.

Coticchio, G., Dal Canto, M., Renzini, M. M., Guglielmo, M. C., Brambillasca, F., Turchi, D., . . . Fadini, R. (2015). Oocyte maturation: gamete-somatic cells interactions, meiotic resumption, cytoskeletal dynamics and cytoplasmic reorganization. Human Reproduction Update, 21(4), 427-454.

Crawford, J. L., Heath, D. A., Reader, K. L., Quirke, L. D., Hudson, N. L., Juengel, J. L., \& McNatty, K. P. (2011). Oocytes in sheep homozygous for a mutation in bone morphogenetic protein receptor $1 \mathrm{~B}$ express lower mRNA levels of bone morphogenetic protein 15 but not growth differentiation factor 9. Reproduction, 142(1), 53-61. 
Crawford, J. L., \& McNatty, K. P. (2012). The ratio of growth differentiation factor 9: Bone morphogenetic protein 15 mRNA expression is tightly co-regulated and differs between species over a wide range of ovulation rates. Molecular and Cellular Endocrinology, 348(1), 339-343.

Crosby, I. M., Gandolfi, F., \& Moor, R. M. (1988). Control of protein synthesis during early cleavage of sheep embryos. Journal of Reproduction and Fertility, 82(2), 769-775.

Davis, G. H., McEwan, J. C., Fennessy, P. F., Dodds, K. G., \& Farquhar, P. A. (1991). Evidence for the presence of a major gene influencing ovulation rate on the $X$ chromosome of sheep. Biology of Reproduction, 44(4), 620-624.

De Vos, M., Smitz, J., Thompson, J. G., \& Gilchrist, R. B. (2016). The definition of IVM is clearvariations need defining. Hum Reprod, 31(11), 2411-2415.

Dhali, A., Javvaji, P. K., Kolte, A. P., Francis, J. R., Roy, S. C., \& Sejian, V. (2017). Temporal expression of cumulus cell marker genes during in vitro maturation and oocyte developmental competence. J Assist Reprod Genet, 34(11), 1493-1500.

Diaz, F. J., Wigglesworth, K., \& Eppig, J. J. (2007). Oocytes are required for the preantral granulosa cell to cumulus cell transition in mice. Dev Biol, 305(1), 300-311.

Donahue, R. P., \& Stern, S. (1968). Follicular cell support of oocyte maturation: production of pyruvate in vitro. Journal of Reproduction and Fertility, 17(2), 395-398.

Dong, J. W., Albertini, D. F., Nishimori, K., Kumar, T. R., Lu, N. F., \& Matzuk, M. M. (1996). Growth differentiation factor-9 is required during early ovarian folliculogenesis. Nature, 383(6600), 531-535. doi:DOI 10.1038/383531a0

Douglas, N., Tang, H., Nakhuda, G. S., Sauer, M., \& Zimmermann, R. (2005). Oocyte expression of hypoxia and hypoxia inducible factors (HIF). Fertility and Sterility, 84, S390-S390.

Driancourt, M. A. G., W.R., Cahill, L.P. (1985). Follicular dynamics throughout the oestrous cycle in sheep. A review. Reprod. Nutr. Dévelop, 25(1A), 1-15.

Dumollard, R., Duchen, M., \& Carroll, J. (2007). The role of mitochondrial function in the oocyte and embryo. Mitochondrion in the Germline and Early Development, 77, 21-+.

Dumollard, R., Duchen, M., \& Sardet, C. (2006). Calcium signals and mitochondria at fertilisation. Seminars in Cell \& Developmental Biology, 17(2), 314-323.

Dumollard, R., Ward, Z., Carroll, J., \& Duchen, M. R. (2007). Regulation of redox metabolism in the mouse oocyte and embryo. Development, 134(3), 455-465. 
Dunning, K. R., Cashman, K., Russell, D. L., Thompson, J. G., Norman, R. J., \& Robker, R. L. (2010). Beta-oxidation is essential for mouse oocyte developmental competence and early embryo development. Biology of Reproduction, 83(6), 909-918.

Dunning, K. R., Lane, M., Brown, H. M., Yeo, C., Robker, R. L., \& Russell, D. L. (2007). Altered composition of the cumulus-oocyte complex matrix during in vitro maturation of oocytes. Hum Reprod, 22(11), 2842-2850.

Dunning, K. R., Russell, D. L., \& Robker, R. L. (2014). Lipids and oocyte developmental competence: the role of fatty acids and beta-oxidation. Reproduction, 148(1), R15-27.

Dunning, K. R., Watson, L. N., Sharkey, D. J., Brown, H. M., Norman, R. J., Thompson, J. G., . . . Russell, D. L. (2012). Molecular Filtration Properties of the Mouse Expanded Cumulus Matrix: Controlled Supply of Metabolites and Extracellular Signals to Cumulus Cells and the Oocyte. Biology of Reproduction, 87(4).

Dyer, S., Chambers, G. M., de Mouzon, J., Nygren, K. G., Zegers-Hochschild, F., Mansour, R., .. . Adamson, G. D. (2016). International Committee for Monitoring Assisted Reproductive Technologies world report: Assisted Reproductive Technology 2008, 2009 and 2010. Hum Reprod, 31(7), 1588-1609.

Eckery, D. C. T., D.J.; Heath, D.A.; McNatty, K.P. . (1996). Morphology and function of the ovary during fetal and early neonatal life: A comparison between the sheep and brushtail possum (Trichosurus vulpecula). Anim Reprod Sci, 42, 551-561.

Edwards, R. G. (1965). Maturation in vitro of mouse, sheep, cow, pig, rhesus monkey and human ovarian oocytes. Nature, 208(5008), 349-351.

Edwards, R. G. (2007). Are minimal stimulation IVF and IVM set to replace routine IVF? Reproductive Biomedicine Online, 14(2), 267-270.

Ekart, J., McNatty, K., Hutton, J., \& Pitman, J. (2013). Ranking and selection of MIl oocytes in human ICSI cycles using gene expression levels from associated cumulus cells. Human Reproduction, 28(11), 2930-2942.

El-Hayek, S., Demeestere, I., \& Clarke, H. J. (2014). Follicle-stimulating hormone regulates expression and activity of epidermal growth factor receptor in the murine ovarian follicle. Proceedings of the National Academy of Sciences of the United States of America, 111(47), 16778-16783. 
Elvin, J. A., Clark, A. T., Wang, P., Wolfman, N. M., \& Matzuk, M. M. (1999). Paracrine actions of growth differentiation factor-9 in the mammalian ovary. Mol Endocrinol, 13(6), 10351048.

Eppig, J. J. (1980). Regulation of Cumulus Oophorus Expansion by Gonadotropins Invivo and Invitro. Biology of Reproduction, 23(3), 545-552.

Eppig, J. J., O'Brien, M. J., Wigglesworth, K., Nicholson, A., Zhang, W., \& King, B. A. (2009). Effect of in vitro maturation of mouse oocytes on the health and lifespan of adult offspring. Hum Reprod, 24(4), 922-928.

Eppig, J. J., Wigglesworth, K., Pendola, F., \& Hirao, Y. (1997). Murine oocytes suppress expression of luteinizing hormone receptor messenger ribonucleic acid by granulosa cells. Biology of Reproduction, 56(4), 976-984.

Erickson, B. H. (1966). Development and senescence of the postnatal bovine ovary. J Anim Sci, 25(3), 800-805.

Erickson, G. F., Wang, C., \& Hsueh, A. J. W. (1979). Fsh Induction of Functional Lh Receptors in Granulosa-Cells Cultured in a Chemically Defined Medium. Nature, 279(5711), 336-338.

Ferreira, E. M., Vireque, A. A., Adona, P. R., Meirelles, F. V., Ferriani, R. A., \& Navarro, P. A. (2009). Cytoplasmic maturation of bovine oocytes: structural and biochemical modifications and acquisition of developmental competence. Theriogenology, 71(5), 836-848.

Feuerstein, P., Cadoret, V., Dalbies-Tran, R., Guerif, F., Bidault, R., \& Royere, D. (2007). Gene expression in human cumulus cells: one approach to oocyte competence. Hum Reprod, 22(12), 3069-3077.

Fissore, R. A., Kurokawa, M., Knott, J., Zhang, M., \& Smyth, J. (2002). Mechanisms underlying oocyte activation and postovulatory ageing. Reproduction, 124(6), 745-754.

Flagg-Newton, J., Simpson, I., \& Loewenstein, W. R. (1979). Permeability of the cell-to-cell membrane channels in mammalian cell juncton. Science, 205(4404), 404-407.

Forde, N., Beltman, M. E., Lonergan, P., Diskin, M., Roche, J. F., \& Crowe, M. A. (2011). Oestrous cycles in Bos taurus cattle. Anim Reprod Sci, 124(3-4), 163-169.

Frank, L. A., Sutton-McDowall, M. L., Brown, H. M., Russell, D. L., Gilchrist, R. B., \& Thompson, J. G. (2014). Hyperglycaemic conditions perturb mouse oocyte in vitro developmental competence via beta-O-linked glycosylation of heat shock protein 90. Hum Reprod, 29(6), 1292-1303. 
Fulop, C., Kamath, R. V., Li, Y., Otto, J. M., Salustri, A., Olsen, B. R., . . Hascall, V. C. (1997). Coding sequence, exon-intron structure and chromosomal localization of murine TNFstimulated gene 6 that is specifically expressed by expanding cumulus cell-oocyte complexes. Gene, 202(1-2), 95-102.

Galloway, S. M., McNatty, K. P., Cambridge, L. M., Laitinen, M. P., Juengel, J. L., Jokiranta, T. S., . .. Ritvos, O. (2000). Mutations in an oocyte-derived growth factor gene (BMP15) cause increased ovulation rate and infertility in a dosage-sensitive manner. Nat Genet, 25(3), 279-283.

Gardner, D. K., Wale, P. L., Collins, R., \& Lane, M. (2011). Glucose consumption of single postcompaction human embryos is predictive of embryo sex and live birth outcome. Human Reproduction, 26(8), 1981-1986.

Gilchrist, R. B., Lane, M., \& Thompson, J. G. (2008). Oocyte-secreted factors: regulators of cumulus cell function and oocyte quality. Hum Reprod Update, 14(2), 159-177.

Gilula, N. B., Epstein, M. L., \& Beers, W. H. (1978). Cell-to-cell communication and ovulation. A study of the cumulus-oocyte complex. J Cell Biol, 78(1), 58-75.

Ginsburg, M., Snow, M. H., \& McLaren, A. (1990). Primordial germ cells in the mouse embryo during gastrulation. Development, 110(2), 521-528.

Ginther, O. J., Beg, M. A., Donadeu, F. X., \& Bergfelt, D. R. (2003). Mechanism of follicle deviation in monovular farm species. Anim Reprod Sci, 78(3-4), 239-257.

Goto, K., Kajihara, Y., Kosaka, S., Koba, M., Nakanishi, Y., \& Ogawa, K. (1988). Pregnancies after Co-Culture of Cumulus Cells with Bovine Embryos Derived from Invitro Fertilization of Invitro Matured Follicular Oocytes. Journal of Reproduction and Fertility, 83(2), 753-758.

Gu, L., Liu, H., Gu, X., Boots, C., Moley, K. H., \& Wang, Q. (2015). Metabolic control of oocyte development: linking maternal nutrition and reproductive outcomes. Cell Mol Life Sci, 72(2), 251-271.

Guerif, F., McKeegan, P., Leese, H. J., \& Sturmey, R. G. (2013). A Simple Approach for COnsumption and RElease (CORE) Analysis of Metabolic Activity in Single Mammalian Embryos. PLoS One, 8(8).

Hagemann, L. J. (1999). Influence of the dominant follicle on oocytes from subordinate follicles. Theriogenology, 51(2), 449-459.

Hanrahan, J. P., Gregan, S. M., Mulsant, P., Mullen, M., Davis, G. H., Powell, R., \& Galloway, S. M. (2004). Mutations in the genes for oocyte-derived growth factors GDF9 and BMP15 
are associated with both increased ovulation rate and sterility in Cambridge and Belclare sheep (Ovis aries). Biology of Reproduction, 70(4), 900-909.

Hardy, K., Robinson, F. M., Paraschos, T., Wicks, R., Franks, S., \& Winston, R. M. (1995). Normal development and metabolic activity of preimplantation embryos in vitro from patients with polycystic ovaries. Hum Reprod, 10(8), 2125-2135.

Hari, J., Weilenmann, R., Stranzinger, G., \& Pliska, V. (1988). Reproductive-Cycles in White Alpine Sheep - Effect of Ram and Shearing. Zuchthygiene-Reproduction in Domestic Animals, 23(2), 82-85.

Harris, S. E., Leese, H. J., Gosden, R. G., \& Picton, H. M. (2009). Pyruvate and oxygen consumption throughout the growth and development of murine oocytes. Mol Reprod Dev, 76(3), 231-238.

Hasegawa, J., Yanaihara, A., Iwasaki, S., Mitsukawa, K., Negishi, M., \& Okai, T. (2007). Reduction of connexin 43 in human cumulus cells yields good embryo competence during ICSI. J Assist Reprod Genet, 24(10), 463-466.

Hasegawa, J., Yanaihara, A., Iwasaki, S., Otsuka, Y., Negishi, M., Akahane, T., \& Okai, T. (2005). Reduction of progesterone receptor expression in human cumulus cells at the time of oocyte collection during IVF is associated with good embryo quality. Hum Reprod, 20(8), 2194-2200.

Hasler, J. F., Henderson, W. B., Hurtgen, P. J., Jin, Z. Q., Mccauley, A. D., Mower, S. A., . . Trimmer, S. A. (1995). Production, Freezing and Transfer of Bovine Ivf Embryos and Subsequent Calving Results. Theriogenology, 43(1), 141-152.

Heath, D. A. (2016). Oocyte-derived forms of ruminant BMP15 and GDF9 and a theoretical model to explain their synergistic response (Ph.D thesis). Victoria University of Wellington.

Heath, D. A., Pitman, J. L., \& McNatty, K. P. (2017). Molecular forms of ruminant BMP15 and GDF9 and putative interactions with receptors. Reproduction, 154(4), 521-534.

Hirshfield, A. N. (1991). Development of follicles in the mammalian ovary. Int Rev Cytol, 124, 43101.

Holtz, W., \& Lindloff, G. (1976). Prostaglandins as an Aid to Control Reproductive Activity in Cattle, Sheep and Pigs .2. Control of Estrous-Cycle. Zentralblatt Fur Veterinarmedizin Reihe a-Journal of Veterinary Medicine Series a-Animal Physiology Pathology and Clinical Veterinary Medicine, 23(7), 539-548. 
Hsieh, M., Zamah, A. M., \& Conti, M. (2009). Epidermal growth factor-like growth factors in the follicular fluid: role in oocyte development and maturation. Semin Reprod Med, 27(1), $52-61$.

Hummitzsch, K., Irving-Rodgers, H. F., Hatzirodos, N., Bonner, W., Sabatier, L., Reinhardt, D. P., . . Rodgers, R. J. (2013). A new model of development of the mammalian ovary and follicles. PLoS One, 8(2), e55578.

Hussein, T. S., Froiland, D. A., Amato, F., Thompson, J. G., \& Gilchrist, R. B. (2005). Oocytes prevent cumulus cell apoptosis by maintaining a morphogenic paracrine gradient of bone morphogenetic proteins. Journal of Cell Science, 118(22), 5257-5268.

Hussein, T. S., Thompson, J. G., \& Gilchrist, R. B. (2006). Oocyte-secreted factors enhance oocyte developmental competence. Dev Biol, 296(2), 514-521.

Jansen, R. P. S., \& de Boer, K. (1998). The bottleneck: mitochondrial imperatives in oogenesis and ovarian follicular fate. Molecular and Cellular Endocrinology, 145(1-2), 81-88.

Jiao, G. Z., Cao, X. Y., Cui, W., Lian, H. Y., Miao, Y. L., Wu, X. F., . . Tan, J. H. (2013).

Developmental potential of prepubertal mouse oocytes is compromised due mainly to their impaired synthesis of glutathione. PLoS One, 8(3), e58018.

Johnson, M. H., \& Nasr-Esfahani, M. H. (1994). Radical solutions and cultural problems: could free oxygen radicals be responsible for the impaired development of preimplantation mammalian embryos in vitro? Bioessays, 16(1), 31-38.

Juengel, J. L., Hudson, N. L., Heath, D. A., Smith, P., Reader, K. L., Lawrence, S. B., . . McNatty, K. P. (2002). Growth differentiation factor 9 and bone morphogenetic protein 15 are essential for ovarian follicular development in sheep. Biology of Reproduction, 67(6), 1777-1789.

Jungheim, E. S., Macones, G. A., Odem, R. R., Patterson, B. W., Lanzendorf, S. E., Ratts, V. S., \& Moley, K. H. (2011). Associations between free fatty acids, cumulus oocyte complex morphology and ovarian function during in vitro fertilization. Fertility and Sterility, 95(6), 1970-1974.

Kaivo-Oja, N., Mottershead, D. G., Mazerbourg, S., Myllymaa, S., Duprat, S., Gilchrist, R. B., . . . Ritvos, O. (2005). Adenoviral gene transfer allows Smad-responsive gene promoter analyses and delineation of type I receptor usage of transforming growth factor-beta family ligands in cultured human granulosa luteal cells. J Clin Endocrinol Metab, 90(1), 271-278. 
Keskintepe, L., Burnley, C. L., \& Brackett, B. G. (1995). Production of Viable Bovine Blastocysts in Defined in-Vitro Conditions. Biology of Reproduction, 52(6), 1410-1417.

Kim, J., Bagchi, I. C., \& Bagchi, M. K. (2009). Signaling by hypoxia-inducible factors is critical for ovulation in mice. Endocrinology, 150(7), 3392-3400.

Knight, P. G., \& Glister, C. (2003). Local roles of TGF-beta superfamily members in the control of ovarian follicle development. Anim Reprod Sci, 78(3-4), 165-183.

Kovacs, G. (2007). Clinical problems associated with ovarian stimulation for conventional IVF. In vitro Maturation of Human Oocytes, 197.

Krisher, R. L. (2004). The effect of oocyte quality on development. J Anim Sci, 82 E-Suppl, E1423.

Lawson, K. A., \& Hage, W. J. (1994). Clonal analysis of the origin of primordial germ cells in the mouse. Ciba Found Symp, 182, 68-84; discussion 84-91.

Leese, H. J. (2002). Quiet please, do not disturb: a hypothesis of embryo metabolism and viability. Bioessays, 24(9), 845-849.

Leyens, G., Verhaeghe, B., Landtmeters, M., Marchandise, J., Knoops, B., \& Donnay, I. (2004). Peroxiredoxin 6 is upregulated in bovine oocytes and cumulus cells during in vitro maturation: Role of intercellular communication. Biology of Reproduction, 71(5), 16461651.

Li, L., Lu, X., \& Dean, J. (2013). The maternal to zygotic transition in mammals. Mol Aspects Med, 34(5), 919-938.

Li, R., \& Albertini, D. F. (2013). The road to maturation: somatic cell interaction and selforganization of the mammalian oocyte. Nature Reviews Molecular Cell Biology, 14(3), 141-152.

Li, R., Norman, R. J., Armstrong, D. T., \& Gilchrist, R. B. (2000). Oocyte-secreted factor(s) determine functional differences between bovine mural granulosa cells and cumulus cells. Biology of Reproduction, 63(3), 839-845.

Lintern-Moore, S., \& Moore, G. P. (1979). The initiation of follicle and oocyte growth in the mouse ovary. Biology of Reproduction, 20(4), 773-778.

Lonergan, P., Monaghan, P., Rizos, D., Boland, M. P., \& Gordon, I. (1994). Effect of follicle size on bovine oocyte quality and developmental competence following maturation, fertilization, and culture in vitro. Mol Reprod Dev, 37(1), 48-53. 
Luciano, A. M., Franciosi, F., Barros, R. G., Dieci, C., \& Lodde, V. (2018). The variable success of in vitro maturation: can we do better? Animal Reproduction, 15(Suppl. 1), 727-736.

Lundy, T. O. C., A., Hudson, N.L., McNatty, K.P. (1999). Populations of granulosa cells in small follicles of the sheep ovary. Journal of Reproduction and Fertility, 115, 251-262.

Macabelli, C. H., Ferreira, R. M., Gimenes, L. U., de Carvalho, N. A. T., Soares, J. G., Ayres, H., .. . Chiaratti, M. R. (2014). Reference Gene Selection for Gene Expression Analysis of Oocytes Collected from Dairy Cattle and Buffaloes during Winter and Summer. PLoS One, 9(3).

Macaulay, A. D., Gilbert, I., Caballero, J., Barreto, R., Fournier, E., Tossou, P., . . Robert, C. (2014). The gametic synapse: RNA transfer to the bovine oocyte. Biology of Reproduction, 91(4), 90.

Mattioli, M., Bacci, M. L., Galeati, G., \& Seren, E. (1989). Developmental competence of pig oocytes matured and fertilized in vitro. Theriogenology, 31(6), 1201-1207.

McDonald, N. Q., \& Hendrickson, W. A. (1993). A structural superfamily of growth factors containing a cystine knot motif. Cell, 73(3), 421-424.

McKeegan, P. J. (2015). Metabolic regulation during early embryo development (Honour's Thesis) Half York Medical School, University of Hull and the University of York.

McMahon, H. E., Hashimoto, O., Mellon, P. L., \& Shimasaki, S. (2008). Oocyte-specific overexpression of mouse bone morphogenetic protein-15 leads to accelerated folliculogenesis and an early onset of acyclicity in transgenic mice. Endocrinology, 149(6), 2807-2815.

McNatty, K. P., Heath, D. A., Hudson, N. L., Reader, K. L., Quirke, L., Lun, S., \& Juengel, J. L. (2010). The conflict between hierarchical ovarian follicular development and superovulation treatment. Reproduction, 140(2), 287-294.

McNatty, K. P., Juengel, J. L., Wilson, T., Galloway, S. M., \& Davis, G. H. (2001). Genetic mutations influencing ovulation rate in sheep. Reprod Fertil Dev, 13(7-8), 549-555.

McNatty, K. P., Moore, L. G., Hudson, N. L., Quirke, L. D., Lawrence, S. B., Reader, K., . . Juengel, J. L. (2004). The oocyte and its role in regulating ovulation rate: a new paradigm in reproductive biology. Reproduction, 128(4), 379-386.

McNatty, K. P., Smith, P., Hudson, N. L., Heath, D. A., Tisdall, D. J., O, W. S., \& Braw-Tal, R. (1995). Development of the sheep ovary during fetal and early neonatal life and the effect of fecundity genes. Bioscientifica Proceedings. 
McPherron, A. C., \& Lee, S. J. (1993). GDF-3 and GDF-9: two new members of the transforming growth factor-beta superfamily containing a novel pattern of cysteines. Journal of Biological Chemistry, 268(5), 3444-3449.

Mehlmann, L. M. (2005). Stops and starts in mammalian oocytes: recent advances in understanding the regulation of meiotic arrest and oocyte maturation. Reproduction, 130(6), 791-799.

Milczewski, V., Chahad-Ehlers, S., Spercoski, K. M., Morais, R. N., \& Soccol, V. T. (2015). Quantifying the effect of seasonality on testicular function of Suffolk ram in lower latitude. Small Ruminant Research, 124, 68-75. doi:10.1016/j.smallrumres.2014.12.012

Misztal, T., Romanowicz, K., \& Barcikowski, B. (2002). Melatonin--a modulator of the GnRH/LH axis in sheep. Reproductive Biology, 2(3), 267-275.

Moore, R. K., Otsuka, F., \& Shimasaki, S. (2003). Molecular basis of bone morphogenetic protein-15 signaling in granulosa cells. Journal of Biological Chemistry, 278(1), 304-310.

Mottershead, D. G., Sugimura, S., Al-Musawi, S. L., Li, J. J., Richani, D., White, M. A., . . Gilchrist, R. B. (2015). Cumulin, an Oocyte-secreted Heterodimer of the Transforming Growth Factor-beta Family, Is a Potent Activator of Granulosa Cells and Improves Oocyte Quality. Journal of Biological Chemistry, 290(39), 24007-24020.

Moura, B. R., Gurgel, M. C., Machado, S. P., Marques, P. A., Rolim, J. R., Lima, M. C., \& Salgueiro, L. L. (2017). Low concentration of hyaluronidase for oocyte denudation can improve fertilization rates and embryo quality. JBRA Assist Reprod, 21(1), 27-30.

Murray L, B. D., McDonald R \& Pitman JL. (2017). Refinement of an IVM system on oocyte developmental competency in New Zealand dairy cows. . Study of Reproduction 50th Annual Meeting, Washington DC, USA.

Murray, L. A. (2020). Refinement of a Physiologically-Relevant IVM/IVF System on Oocyte Developmental Competency in New Zealand Dairy Cows (Ph.D. thesis). Victoria Universiy of Wellington.

Pangas, S. A., Jorgez, C. J., \& Matzuk, M. M. (2004). Growth differentiation factor 9 regulates expression of the bone morphogenetic protein antagonist gremlin. Journal of Biological Chemistry, 279(31), 32281-32286.

Paradis, F., Novak, S., Murdoch, G. K., Dyck, M. K., Dixon, W. T., \& Foxcroft, G. R. (2009). Temporal regulation of BMP2, BMP6, BMP15, GDF9, BMPR1A, BMPR1B, BMPR2 and 
TGFBR1 mRNA expression in the oocyte, granulosa and theca cells of developing preovulatory follicles in the pig. Reproduction, 138(1), 115-129.

Park, J. Y., Su, Y. Q., Ariga, M., Law, E., Jin, S. L. C., \& Conti, M. (2004). EGF-like growth factors as mediators of LH action in the ovulatory follicle. Science, 303(5658), 682-684.

Paulini, F., \& Melo, E. O. (2011). The role of oocyte-secreted factors GDF9 and BMP15 in follicular development and oogenesis. Reprod Domest Anim, 46(2), 354-361.

Pincus, G., \& Enzmann, E. V. (1935). The Comparative Behavior of Mammalian Eggs in Vivo and in Vitro : I. The Activation of Ovarian Eggs. J Exp Med, 62(5), 665-675.

Pulkki, M. M., Mottershead, D. G., Pasternack, A. H., Muggalla, P., Ludlow, H., van Dinther, M., . .. Ritvos, O. (2012). A covalently dimerized recombinant human bone morphogenetic protein-15 variant identifies bone morphogenetic protein receptor type $1 \mathrm{~B}$ as a key cell surface receptor on ovarian granulosa cells. Endocrinology, 153(3), 1509-1518.

Rabahi, F., Brule, S., Sirois, J., Beckers, J. F., Silversides, D. W., \& Lussier, J. G. (1999). High expression of bovine alpha glutathione S-transferase (GSTA1, GSTA2) subunits is mainly associated with steroidogenically active cells and regulated by gonadotropins in bovine ovarian follicles. Endocrinology, 140(8), 3507-3517.

Rawe, V. Y., \& Combelles, C. M. H. (2009). Human Oocyte Abnormalities: Basic Analyses and Clinical Applications. In Biennial Review of Infertility (pp. 193-214).

Reader, K. L., Heath, D. A., Lun, S., McIntosh, C. J., Western, A. H., Littlejohn, R. P., . . Juengel, J. L. (2011). Signalling pathways involved in the cooperative effects of ovine and murine GDF9+BMP15-stimulated thymidine uptake by rat granulosa cells. Reproduction, 142(1), 123-131.

Reader, K. L., Mottershead, D. G., Martin, G. A., Gilchrist, R. B., Heath, D. A., McNatty, K. P., \& Juengel, J. L. (2016). Signalling pathways involved in the synergistic effects of human growth differentiation factor 9 and bone morphogenetic protein 15. Reprod Fertil Dev, 28(4), 491-498.

Reinhardt, R. R., Chin, E., Zhou, J., Taira, M., Murata, T., Manganiello, V. C., \& Bondy, C. A. (1995). Distinctive anatomical patterns of gene expression for cGMP-inhibited cyclic nucleotide phosphodiesterases. J Clin Invest, 95(4), 1528-1538.

Richani, D., Ritter, L. J., Thompson, J. G., \& Gilchrist, R. B. (2013). Mode of oocyte maturation affects EGF-like peptide function and oocyte competence. Molecular Human Reproduction, 19(8), 500-509. 
Richani, D., Wang, X., Zeng, H. T., Smitz, J., Thompson, J. G., \& Gilchrist, R. B. (2014). Prematuration with cAMP modulators in conjunction with EGF-like peptides during in vitro maturation enhances mouse oocyte developmental competence. Mol Reprod Dev, 81(5), 422-435.

Robker, R. L., Akison, L. K., \& Russell, D. L. (2009). Control of oocyte release by progesterone receptor-regulated gene expression. Nucl Recept Signal, 7, e012.

Rodgers, R. J., \& Irving-Rodgers, H. F. (2010). Formation of the ovarian follicular antrum and follicular fluid. Biology of Reproduction, 82(6), 1021-1029.

Saccon, R. A., Bunton-Stasyshyn, R. K. A., Fisher, E. M. C., \& Fratta, P. (2013). Is SOD1 loss of function involved in amyotrophic lateral sclerosis? Brain, 136, 2342-2358.

Salhab, M., Dhorne-Pollet, S., Auclair, S., Guyader-Joly, C., Brisard, D., Dalbies-Tran, R., . . . Uzbekova, S. (2013). In vitro maturation of oocytes alters gene expression and signaling pathways in bovine cumulus cells. Mol Reprod Dev, 80(2), 166-182.

Salhab, M., Tosca, L., Cabau, C., Papillier, P., Perreau, C., Dupont, J., . . . Uzbekova, S. (2011). Kinetics of gene expression and signaling in bovine cumulus cells throughout IVM in different mediums in relation to oocyte developmental competence, cumulus apoptosis and progesterone secretion. Theriogenology, 75(1), 90-104.

Santiquet, N. W., Greene, A. F., Becker, J., Barfield, J. P., Schoolcraft, W. B., \& Krisher, R. L. (2017). A pre-in vitro maturation medium containing cumulus oocyte complex ligandreceptor signaling molecules maintains meiotic arrest, supports the cumulus oocyte complex and improves oocyte developmental competence. Mol Hum Reprod, 23(9), 594606.

Sasseville, M., Gagnon, M. C., Guillemette, C., Sullivan, R., Gilchrist, R. B., \& Richard, F. J. (2009). Regulation of gap junctions in porcine cumulus-oocyte complexes: contributions of granulosa cell contact, gonadotropins, and lipid rafts. Mol Endocrinol, 23(5), 700-710.

Sawyer, H. R., Smith, P., Heath, D. A., Juengel, J. L., Wakefield, S. J., \& McNatty, K. P. (2002). Formation of ovarian follicles during fetal development in sheep. Biology of Reproduction, 66(4), 1134-1150.

Scaramuzzi, R. J., \& Radford, H. M. (1983). Factors Regulating Ovulation Rate in the Ewe. Journal of Reproduction and Fertility, 69(1), 353-367.

Schanbacher, B. D. (1978). Fertility of Rams Chronically Treated with Gonadotropin-Releasing Hormone during Non-Breeding Season. Biology of Reproduction, 19(3), 661-665. 
Simon, A. M., Goodenough, D. A., Li, E., \& Paul, D. L. (1997). Female infertility in mice lacking connexin 37. Nature, 385(6616), 525-529. doi:10.1038/385525a0

Sinclair, K. D., Lunn, L. A., Kwong, W. Y., Wonnacott, K., Linforth, R. S. T., \& Craigon, J. (2008). Amino acid and fatty acid composition of follicular fluid as predictors of in-vitro embryo development. Reproductive Biomedicine Online, 16(6), 859-868.

Sirard, M. A., Parrish, J. J., Ware, C. B., Leibfriedrutledge, M. L., \& First, N. L. (1988). The Culture of Bovine Oocytes to Obtain Developmentally Competent Embryos. Biology of Reproduction, 39(3), 546-552.

Sirard, M. A., Richard, F., Blondin, P., \& Robert, C. (2006). Contribution of the oocyte to embryo quality. Theriogenology, 65(1), 126-136.

Sirois, J., \& Fortune, J. E. (1988). Ovarian follicular dynamics during the estrous cycle in heifers monitored by real-time ultrasonography. Biology of Reproduction, 39(2), 308-317.

Son, W. Y., Henderson, S., Cohen, Y., Dahan, M., \& Buckett, W. (2019). Immature Oocyte for Fertility Preservation. Front Endocrinol (Lausanne), 10, 464.

Souza, C. J. H., Campbell, B. K., \& Baird, D. T. (1997). Follicular dynamics and ovarian steroid secretion in sheep during the follicular and early luteal phases of the estrous cycle. Biology of Reproduction, 56(2), 483-488.

Su, Y. Q., Wu, X. M., O'Brien, M. J., Pendola, F. L., Denegre, J. N., Matzuk, M. M., \& Eppig, J. J. (2004). Synergistic roles of BMP15 and GDF9 in the development and function of the oocyte-cumulus cell complex in mice: genetic evidence for an oocyte-granulosa cell regulatory loop. Developmental Biology, 276(1), 64-73.

Sudiman, J., Sutton-McDowall, M. L., Ritter, L. J., White, M. A., Mottershead, D. G., Thompson, J. G., \& Gilchrist, R. B. (2014). Bone morphogenetic protein 15 in the pro-mature complex form enhances bovine oocyte developmental competence. PLoS One, 9(7), e103563.

Sugimura, S., Kobayashi, N., Okae, H., Yamanouchi, T., Matsuda, H., Kojima, T., . . Gilchrist, R. B. (2017). Transcriptomic signature of the follicular somatic compartment surrounding an oocyte with high developmental competence. Sci Rep, 7(1), 6815.

Sugimura, S., Ritter, L. J., Rose, R. D., Thompson, J. G., Smitz, J., Mottershead, D. G., \& Gilchrist, R. B. (2015). Promotion of EGF receptor signaling improves the quality of low developmental competence oocytes. Dev Biol, 403(2), 139-149. 
Sugiura, K., \& Eppig, J. J. (2005). Society for Reproductive Biology Founders' Lecture 2005.

Control of metabolic cooperativity between oocytes and their companion granulosa cells by mouse oocytes. Reprod Fertil Dev, 17(7), 667-674.

Sun, P. D., \& Davies, D. R. (1995). The cystine-knot growth-factor superfamily. Annu Rev Biophys Biomol Struct, 24, 269-291.

Sutton, M. L., Gilchrist, R. B., \& Thompson, J. G. (2003). Effects of in-vivo and in-vitro environments on the metabolism of the cumulus-oocyte complex and its influence on oocyte developmental capacity. Human Reproduction Update, 9(1), 35-48.

Sutton-McDowall, M. L., Gilchrist, R. B., \& Thompson, J. G. (2010). The pivotal role of glucose metabolism in determining oocyte developmental competence. Reproduction, 139(4), 685-695.

Swinerd, G. W. (2016). The molecular forms of BMP15 in a range of mammalian species (Master's thesis). Victoria University of Wellington.

Takahashi, N., Davy, P. M. C., Gardner, L. H., Mathews, J., Yamazaki, Y., \& Allsopp, R. C. (2016). Hypoxia Inducible Factor 1 Alpha Is Expressed in Germ Cells throughout the Murine Life Cycle. PLoS One, 11(5).

Tarin, J. J. (1996). Potential effects of age-associated oxidative stress on mammalian oocytes/embryos. Mol Hum Reprod, 2(10), 717-724. doi:10.1093/molehr/2.10.717

Tennant, A. H., \& Kligerman, A. D. (2011). Superoxide Dismutase Protects Cells From DNA Damage Induced by Trivalent Methylated Arsenicals. Environmental and Molecular Mutagenesis, 52(3).

Thomas, R. E., Armstrong, D. T., \& Gilchrist, R. B. (2004). Bovine cumulus cell-oocyte gap junctional communication during in vitro maturation in response to manipulation of cellspecific cyclic adenosine 3',5'-monophosophate levels. Biology of Reproduction, 70(3), 548-556.

Tripathi, A., Kumar, K. V. P., \& Chaube, S. K. (2010). Meiotic Cell Cycle Arrest in Mammalian Oocytes. Journal of Cellular Physiology, 223(3), 592-600.

Trounson, A., Wood, C., \& Kausche, A. (1994). In-Vitro Maturation and the Fertilization and Developmental Competence of Oocytes Recovered from Untreated Polycystic Ovarian Patients. Fertility and Sterility, 62(2), 353-362.

Tsafriri, A., Chun, S. Y., Zhang, R., Hsueh, A. J., \& Conti, M. (1996). Oocyte maturation involves compartmentalization and opposing changes of cAMP levels in follicular somatic and 
germ cells: studies using selective phosphodiesterase inhibitors. Dev Biol, 178(2), 393402.

Tsafriri, A., Pomerantz, S. H., \& Channing, C. P. (1976). Inhibition of oocyte maturation by porcine follicular fluid: partial characterization of the inhibitor. Biology of Reproduction, 14(5), 511-516.

Tsafriri, A., Zor, U., Lamprecht, S. A., \& Lindner, H. R. (1972). In-Vitro Induction of Meiotic Division in Follicle-Enclosed Rat Oocytes by Lh, Cyclic Amp and Prostaglandin-E2. Journal of Reproduction and Fertility, 31(1), 39-+.

Ublacker, G. A., Johnson, J. A., Siegel, F. L., \& Mulcahy, R. T. (1991). Influence of glutathione Stransferases on cellular glutathione determination by flow cytometry using monochlorobimane. Cancer Res, 51(7), 1783-1788.

Uhde, K., van Tol, H. T. A., Stout, T. A. E., \& Roelen, B. A. J. (2018). Metabolomic profiles of bovine cumulus cells and cumulus-oocyte-complex-conditioned medium during maturation in vitro. Sci Rep, 8(1), 9477.

Urman, B., Fluker, M. R., Yuen, B. H., Fleige-Zahradka, B. G., Zouves, C. G., \& Moon, Y. S. (1992). The outcome of in vitro fertilization and embryo transfer in women with polycystic ovary syndrome failing to conceive after ovulation induction with exogenous gonadotropins. Fertility and Sterility, 57(6), 1269-1273.

Urner, F., \& Sakkas, D. (1999). A possible role for the pentose phosphate pathway of spermatozoa in gamete fusion in the mouse. Biology of Reproduction, 60(3), 733-739.

van Tol, H. T., van Eijk, M. J., Mummery, C. L., van den Hurk, R., \& Bevers, M. M. (1996). Influence of FSH and hCG on the resumption of meiosis of bovine oocytes surrounded by cumulus cells connected to membrana granulosa. Mol Reprod Dev, 45(2), 218-224.

Vanderhyden, B. C., \& Armstrong, D. T. (1989). Role of Cumulus Cells and Serum on the Invitro Maturation, Fertilization, and Subsequent Development of Rat Oocytes. Biology of Reproduction, 40(4), 720-728.

Virant-Klun, I., Knez, K., Tomazevic, T., \& Skutella, T. (2013). Gene expression profiling of human oocytes developed and matured in vivo or in vitro. Biomed Res Int, 2013, 879489.

Vitt, U. A., Mazerbourg, S., Klein, C., \& Hsueh, A. J. (2002). Bone morphogenetic protein receptor type II is a receptor for growth differentiation factor-9. Biology of Reproduction, $67(2), 473-480$. 
Walls, M. L., Hunter, T., Ryan, J. P., Keelan, J. A., Nathan, E., \& Hart, R. J. (2015). In vitro maturation as an alternative to standard in vitro fertilization for patients diagnosed with polycystic ovaries: a comparative analysis of fresh, frozen and cumulative cycle outcomes. Human Reproduction, 30(1), 88-96.

Ward, M. L. (2018). Investigating the effect of a physiologically relevant IVM system on the function of bovine cumulus cell-oocyte complexes (Master's thesis). Victoria University of Wellington.

Webb, R., \& England, B. G. (1982). Identification of the ovulatory follicle in the ewe: associated changes in follicular size, thecal and granulosa cell luteinizing hormone receptors, antral fluid steroids, and circulating hormones during the preovulatory period. Endocrinology, 110(3), 873-881.

Wiesen, J. F., \& Midgley, A. R., Jr. (1993). Changes in expression of connexin 43 gap junction messenger ribonucleic acid and protein during ovarian follicular growth. Endocrinology, 133(2), 741-746.

Yamashita, Y., Okamoto, M., Kawashima, I., Okazaki, T., Nishimura, R., Gunji, Y., . . Shimada, M. (2011). Positive feedback loop between prostaglandin E2 and EGF-like factors is essential for sustainable activation of MAPK3/1 in cumulus cells during in vitro maturation of porcine cumulus oocyte complexes. Biology of Reproduction, 85(5), 1073-1082.

Yan, C., Wang, P., DeMayo, J., DeMayo, F. J., Elvin, J. A., Carino, C., . . Matzuk, M. M. (2001). Synergistic roles of bone morphogenetic protein 15 and growth differentiation factor 9 in ovarian function. Mol Endocrinol, 15(6), 854-866.

Yang, H. W., Hwang, K. J., Kwon, H. C., Kim, H. S., Choi, K. W., \& Oh, K. S. (1998). Detection of reactive oxygen species (ROS) and apoptosis in human fragmented embryos. Hum Reprod, 13(4), 998-1002.

Yang, L., Wei, Q., Li, W., Xi, Q., Zhao, X., \& Ma, B. (2016). NPR2 is involved in FSH-mediated mouse oocyte meiotic resumption. J Ovarian Res, 9, 6.

Yoshida, M., Mizoguchi, Y., Ishigaki, K., Kojima, T., \& Nagai, T. (1993). Birth of Piglets Derived from Invitro Fertilization of Pig Oocytes Matured Invitro. Theriogenology, 39(6), 13031311.

Zamboni, L. (1974). Fine morphology of the follicle wall and follicle cell-oocyte association. Biology of Reproduction, 10(2), 125-149. 
Zamboni, L., Bezard, J., \& Mauleon, P. (1979). Role of the Mesonephros in the Development of the Sheep Fetal Ovary. Annales De Biologie Animale Biochimie Biophysique, 19(Nb4), 1153-1178.

Zhai, B., Liu, H., Li, X., Dai, L., Gao, Y., Li, C., .. . Zhang, J. (2013). BMP15 prevents cumulus cell apoptosis through CCL2 and FBN1 in porcine ovaries. Cell Physiol Biochem, 32(2), 264278.

Zhang, M., Su, Y. Q., Sugiura, K., Xia, G., \& Eppig, J. J. (2010). Granulosa cell ligand NPPC and its receptor NPR2 maintain meiotic arrest in mouse oocytes. Science, 330(6002), 366-369.

Zhang, M. J., Hong, O. Y., \& Xia, G. L. (2009). The signal pathway of gonadotrophins-induced mammalian oocyte meiotic resumption. Molecular Human Reproduction, 15(7), 399-409.

Zhang, Q., Sun, H. X., Jiang, Y., Ding, L. J., Wu, S. G., Fang, T., ... Hu, Y. L. (2013). MicroRNA-181a Suppresses Mouse Granulosa Cell Proliferation by Targeting Activin Receptor IIA. PLoS One, 8(3).

10.1371/journal.pone.0059667

Zhu, J., Moawad, A. R., Wang, C. Y., Li, H. F., Ren, J. Y., \& Dai, Y. F. (2018). Advances in in vitro production of sheep embryos. Int J Vet Sci Med, 6(Suppl), S15-S26. 

